\title{
Resolution of the Acroteriobatus leucospilus species complex, with a redescription of $A$. leucospilus (Norman, 1926) and descriptions of two new western Indian Ocean species of Acroteriobatus (Rhinopristiformes, Rhinobatidae)
}

\author{
Simon Weigmann ${ }^{1,2}$ (D) David A. Ebert ${ }^{3,4,5}$ (D) Bernard Séret ${ }^{6}$ (D) \\ Received: 8 December 2020 / Revised: 8 March 2021 / Accepted: 10 March 2021 / Published online: 21 June 2021 \\ (C) The Author(s) 2021
}

\begin{abstract}
Recent sampling efforts and examinations of museum material provided evidence for a complex of species within Acroteriobatus leucospilus (Norman, 1926). The present manuscript contains a redescription of A. leucospilus involving the syntypes and additional material, as well as formal descriptions of two new species of Acroteriobatus Giltay, 1928. All specimens of both new species were found in the western Indian Ocean. Individuals of the first new species, hereafter referred to as Acroteriobatus andysabini sp. nov., were identified originating from Madagascar, and specimens of the second new species, hereafter referred to as Acroteriobatus stehmanni sp. nov., were only found off Socotra Islands at the junction between the Gulf of Aden and the Arabian Sea. Both new species appear to be endemic to the respective region and allopatric to A. leucospilus and occur in coastal waters to at least $80 \mathrm{~m}$ and $43 \mathrm{~m}$ depth, respectively. They differ from A. leucospilus in a number of characteristics including the maximum size and coloration in fresh. Acroteriobatus andysabini sp. nov. is a large species growing to more than $100 \mathrm{~cm}$ total length (TL) and with elongated bluish-gray spots on snout giving "stripe-nosed" appearance; numerous small bluish-gray spots on pectoral, pelvic, dorsal, and caudal fins; brown spots on trunk and fin bases; lateral tail folds striped orange and white; and ventral surface largely white but with a V-shape pattern of faint to dark speckled black spots on snout tip. Acroteriobatus stehmanni sp. nov. is a small species growing to $\sim 62 \mathrm{~cm}$ TL and with sparse patterning with small bluish-gray circular spots confined to snout tip, posterior pectoral-fin margins, a pair on midbody, and few on posterior pelvic-fin margins, rather indistinct small to larger dark brown spots, and lateral tail folds and ventral surface white. Acroteriobatus leucospilus is a medium-sized species growing to $\sim 96 \mathrm{~cm}$ TL and with patterning similar to A. andysabini sp. nov. but ventral surface uniformly white and lateral tail folds white or striped blue and brown. Taxonomical differences include nasal lamellae counts (42-48 in A. andysabini sp. nov. vs. $43-48$ in A. stehmanni sp. nov. vs. 37-41 in A. leucospilus), snout angle (76-85 $5^{\circ}$ vs. $71-77^{\circ}$ vs. $\left.68-81^{\circ}\right)$, and dorsal head length $(24.2-$ $33.5 \%$ vs. $17.2-22.8 \%$ TL vs. $24.0-29.2 \%$ TL). A key to the species of Acroteriobatus is given for the first time.
\end{abstract}

Keywords Chondrichthyes $\cdot$ Elasmobranchii $\cdot$ Systematics $\cdot$ Guitarfishes $\cdot$ Taxonomy $\cdot$ Morphology

This article is registered in ZooBank under http://zoobank.org/ A2D68C03-7D2A-4F8F-82B2-A06CB6A8A2C1

This article is a contribution to the Topical Collection Systematics and Biodiversity of Indian Ocean Sharks, Rays, and Chimaeras (Chondrichthyes).

Communicated by W. White

Simon Weigmann

simon.weigmann@elasmo-lab.de; simon.weigmann@uni-

hamburg.de

1 Elasmo-Lab, Elasmobranch Research Laboratory, Georg-Bonne-Str. 83, 22609 Hamburg, Germany

2 Center of Natural History, University of Hamburg, Martin-Luther-King-Platz 3, 20146 Hamburg, Germany
3 Pacific Shark Research Center, Moss Landing Marine Laboratories, Moss Landing, CA 95039, USA

4 South African Institute for Aquatic Biodiversity, Private Bag 1015, Grahamstown 6140, South Africa

5 Department of Ichthyology, California Academy of Sciences, 55 Music Concourse Drive, San Francisco, CA 94118, USA

6 IchtyoConsult, 6 bis rue du Centre, 91430, Igny, France 


\section{Introduction}

The orders Pristiformes, Rhiniformes, and Rhinobatiformes (sensu Weigmann 2016) were recently revisited by Last et al. (2016a) and combined in the newly described order Rhinopristiformes based on molecular and morphological data, including the families Pristidae and Rhinidae and two new families: the Glaucostegidae and Trygonorrhinidae. Prior to this revision, the Rhinobatidae consisted of six genera, one of which was considered to be highly questionable, and 48 species, including five questionably valid species (Weigmann 2016). The revised family Rhinobatidae contains three genera: Acroteriobatus Giltay, 1928 with eight; Pseudobatos Last, Séret \& Naylor, 2016a with nine; and Rhinobatos Linck, 1790 with 18 species (numbers updated from Last et al. 2016a and Weigmann 2016, 2017). Species of Acroteriobatus are mostly confined to the western Indian Ocean with seven of the eight valid species occurring in this area and only two species also being recorded from the southeastern Atlantic. Species now assigned to Pseudobatos are restricted to the amphiAmerican region, while Rhinobatos is wider ranging, occurring mostly in the Indo-Western Pacific and eastern Atlantic (updated from Weigmann 2016). The three genera can be separated from each other externally by the morphology of their nasal flaps and molecular data (Last et al. 2016a). Species of Acroteriobatus attain maximum sizes of $601-1400 \mathrm{~mm}$ total length (TL) and are found in 0-366 m (usually shallower than $185 \mathrm{~m}$ ) depths (updated from Weigmann 2016). The maximum size of $2050 \mathrm{~mm} \mathrm{TL}$, indicated for Acroteriobatus zanzibarensis (Norman, 1926) by Weigmann (2016) and Séret et al. (2016), was based on an erroneous size indication for the paratype in the original description of the species. The actual size of the paratype is $\sim 650 \mathrm{~mm}$ TL.

During the course of a broader study on western Indian Ocean guitarfishes, an undescribed species of Acroteriobatus was discovered from off Madagascar. Most of the paratypes of this new species were collected aboard the fishing vessel (FV) NOSY BE 11 during the Atimo Vatae expedition (see Bouchet 2014 for details). The Atimo Vatae (meaning "Deep South" in Antandroy language) expedition took place between April and June 2010. It involved a shore-based party of 40 scientists, volunteers, and trainees operating successively from Fort Dauphin (Tolagnaro) between 27 April and 17 May, and from Lavanono between 23 May and 13 June, collecting intertidally, with SCUBA and light dredging. Additionally, Institut de Recherche pour le Développement (IRD) deployed its research catamaran Antéa for sampling by diving, and a chartered shrimp trawler, FV NOSY BE 11, operated by Société des Pêcheries de Nossi Bé (Groupe Unima), was used to sample by dredging and trawling, conducting 119 hauls at 50-700 m depths. Another undescribed species of the genus was found while examining Acroteriobatus material from the northwestern Indian Ocean, collected by Matthias F.W. Stehmann during the $17^{\text {th }}$ cruise of the Russian RV Vityaz in 1988 and 1989. In order to characterize the true Acroteriobatus leucospilus (Norman, 1926), the two syntypes of the species at BMNH were examined. Data of further, more recently collected material was added for a redescription of the species provided herein and to enable delimitation of two previously undescribed species, formally described in the present paper as well. A key to the species of Acroteriobatus is given for the first time.

\section{Material and methods}

Institutional acronyms follow Sabaj (2019) except for HMNH NASU for the National Museum of Natural History, National Academy of Sciences of Ukraine (NASU), Kyiv (Kiev). External morphometric measurements were taken by a vernier caliper to one-tenth of a millimeter $(\mathrm{mm})$ from the specimens preserved in $70 \%$ ethanol. Measurements were taken between perpendicular lines where relevant unless stated otherwise and largely follow Last et al. (2004, 2016a) and Ebert and Gon (2017). The following measurements were newly added in the present paper: snout length (pre-eyeball) measured directly and horizontally, skeletal interorbital width complemented by intereye width measured across mid-eye when it first becomes visible, widths of second and fourth gill openings, body depths at anterior orbit and caudal peduncle, lengths of inner and outer spiracular folds, and distance between bases of folds. Presocket snout length was measured as the direct length from the snout tip to the firm part of the nasal capsule adjacent the orbit (forward of eye socket), spiracle length was defined as the greatest length of the main cavity, preoral length was measured as the direct length from the snout to the posterior edge of upper jaw at its symphysis, mouth width was taken across the exposed width, and pelvic-fin insertion to dorsal-fin origin was defined as the horizontal distance from the pelvic insertion to the origin of the first dorsal fin. Skeletal morphometrics and meristics were taken and counted from radiographs. The description of the new species from off Socotra Islands considers brief field notes and color photographs of fresh specimens, kindly provided by Matthias F.W. Stehmann (ICHTHYS).

The map with catch locations of the examined specimens of all three species of the Acroteriobatus leucospilus species complex was generated based on the Global Relief Model ETOPO1 by NOAA, the National Oceanic and Atmospheric Administration (Amante and Eakins 2009). Country borders, lakes, and rivers were visualized by means of the shapefiles supplied by ESRI for the ArcExplorer-Java Edition for Education 2.3.2 (AEJEE). For a map with all stations of cruise 17 of RV Vityaz, see Weigmann et al. (2013) or Weigmann et al. (2015). 


\section{Comparative material examined}

Acroteriobatus zanzibarensis: BMNH 1868.5.30.4, holotype, adult male, $762 \mathrm{~mm}$ TL, off Zanzibar, Tanzania, western Indian Ocean; BMNH 1867.3.9.429, paratype (dry), adult male, $\sim 650 \mathrm{~mm}$ TL (size indication of $2050 \mathrm{~mm}$ TL in the original description is erroneous), off Zanzibar, Tanzania, western Indian Ocean; ERB 1088, juvenile male, $430 \mathrm{~mm}$ TL, off Kenya; ERB 1117, adult female, $790 \mathrm{~mm}$ TL, off Kenya; ERB 1118, adult female, 736 mm TL, off Kenya.

\section{Results}

\section{Systematic account}

Family Rhinobatidae Bonaparte 1835

Genus Acroteriobatus Giltay, 1928

Type species: Rhinobatus (Syrrhina) annulatus Smith in Müller \& Henle, 1841 by subsequent designation

\section{Acroteriobatus andysabini sp. nov. Weigmann, Ebert \& Séret} http://zoobank.org/0D7EA76E-5268-47D2-882B-866E9FA8BAE4 (Malagasy blue-spotted guitarfish)

(Figs. 1, 2, 3, 4, 5, 6, 7, 8, 9, 10, 11, 12, and 13, Table 1)

Rhinobatus annulatus-Fourmanoir (1963: 35).

Rhinobatos annulatus-Maugé (1967: 217).

Acroteriobatus leucospilus-Fricke et al. (2018: 23).

Acroteriobatus leucospilus-Ghilardi et al. (2019).

The holotype and seven paratypes are deposited in the South African Institute for Aquatic Biodiversity (SAIAB); three paratypes in the Muséum national d'Histoire naturelle, Paris (MNHN); two paratypes in the American Museum of Natural History, New York (AMNH); and one paratype in each of the California Academy of Sciences (CAS) and National Museum of Natural History, Smithsonian Institution, Washington D.C. (USNM).

Holotype SAIAB 97396, $565 \mathrm{~mm}$ TL (fresh), $550 \mathrm{~mm}$ TL (after preservation), juvenile male, Douane Beach, Fort Dauphin, Anosy, southeastern Madagascar, $25^{\circ} 1.651^{\prime} \mathrm{S}$, $46^{\circ} 59.736^{\prime}$ E, collectors E. Heemstra, T. Razafimanambina, and P.C. Heemstra, 27 April 2010.

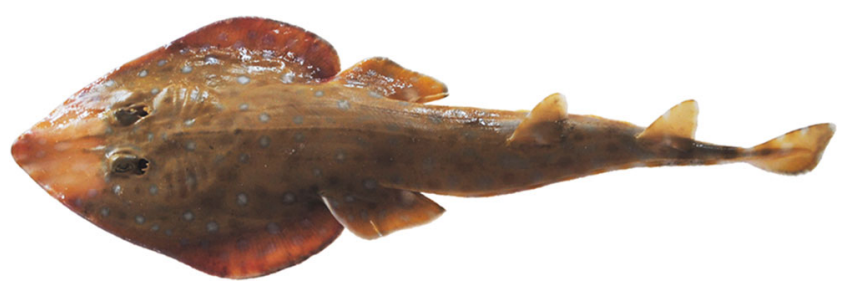

Fig. 1 Acroteriobatus andysabini sp. nov., SAIAB 97396, juvenile male holotype, $565 \mathrm{~mm}$ TL fresh, in total dorsal view taken directly after catching. Photograph by Elaine Heemstra, NRF-SAIAB

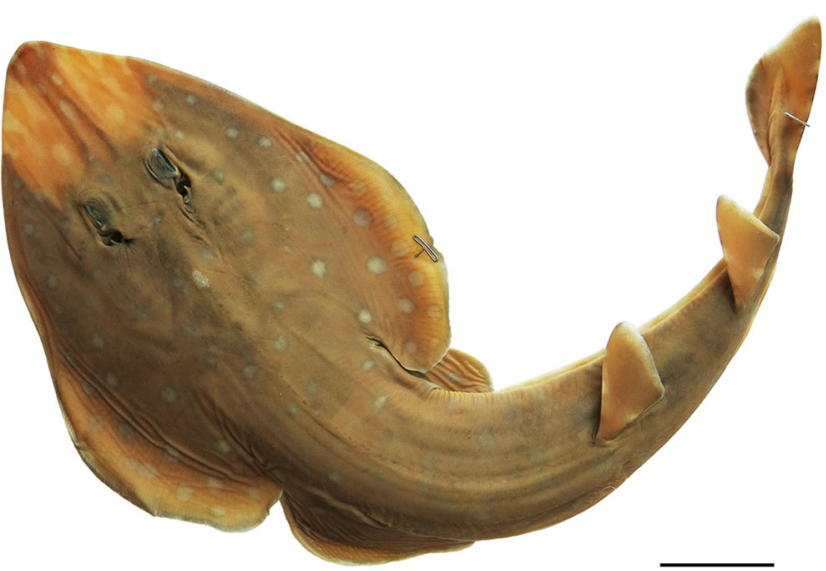

Fig. 2 Acroteriobatus andysabini sp. nov., SAIAB 97396, juvenile male holotype, $550 \mathrm{~mm}$ TL, in total dorsal view. Scale bar: $5 \mathrm{~cm}$. Photograph courtesy Marsha Englebrecht (C)

Paratypes (14) SAIAB 189672, $200 \mathrm{~mm}$ TL (after preservation), juvenile male, Secteur de Fort Dauphin, southeastern Madagascar, $25^{\circ} 5.5^{\prime} \mathrm{S}, 47^{\circ} 5.5^{\prime} \mathrm{E}, 69-70 \mathrm{~m}$ depth, FV NOSY $B E$ 11, station CP 3569, beam trawl, 8 May 2010; SAIAB 189683, $178 \mathrm{~mm}$ TL (after preservation), juvenile male, Secteur de Fort Dauphin, southeastern Madagascar, $25^{\circ} 4.7^{\prime} \mathrm{S}$, $47^{\circ} 3.4^{\prime}$ E, 64-65 m depth, FV NOSY BE 11, station CP 3568, beam trawl, 8 May 2010; SAIAB 189815 (2 specimens), $215 \mathrm{~mm}$ TL (after preservation), juvenile male and $345 \mathrm{~mm}$ TL (after preservation), juvenile female, Secteur de Fort Dauphin, southeastern Madagascar, $25^{\circ} 14.6^{\prime} \mathrm{S}, 47^{\circ} 9.1^{\prime} \mathrm{E}$, 79-80 m depth, FV NOSY BE 11, station CP 3510, beam trawl, 29 April 2010; SAIAB 189816, $602 \mathrm{~mm}$ TL (fresh), $595 \mathrm{~mm}$ TL (after preservation), juvenile male, Sud-Ouest Cap Andavaka, southeastern Madagascar, $25^{\circ} 16.9^{\prime} \mathrm{S}, 46^{\circ} 31.3^{\prime} \mathrm{E}, 53-54 \mathrm{~m}$ depth, FV NOSY BE 11, station CP 3549, beam trawl, 4 May 2010; SAIAB 189943, 195 mm TL (after preservation), juvenile male, Secteur de Fort Dauphin, southeastern Madagascar, $25^{\circ} 6.6^{\prime} \mathrm{S}, 47^{\circ} 6.9^{\prime} \mathrm{E}, 75 \mathrm{~m}$ depth, FV NOSY BE 11, station CP 3570, beam trawl, 8 May 2010; SAIAB 205733,

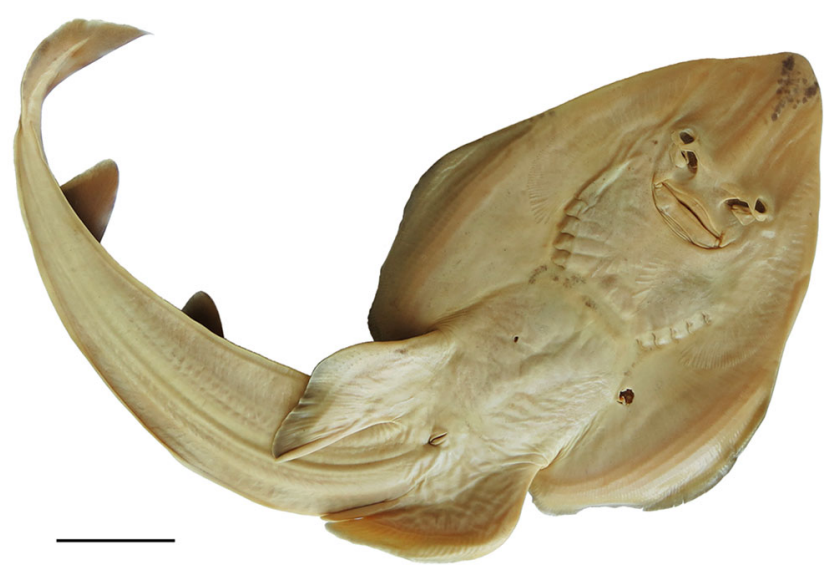

Fig. 3 Acroteriobatus andysabini sp. nov., SAIAB 97396, juvenile male holotype, $550 \mathrm{~mm}$ TL, in total ventral view. Scale bar: $5 \mathrm{~cm}$. Photograph courtesy Marsha Englebrecht (C) 


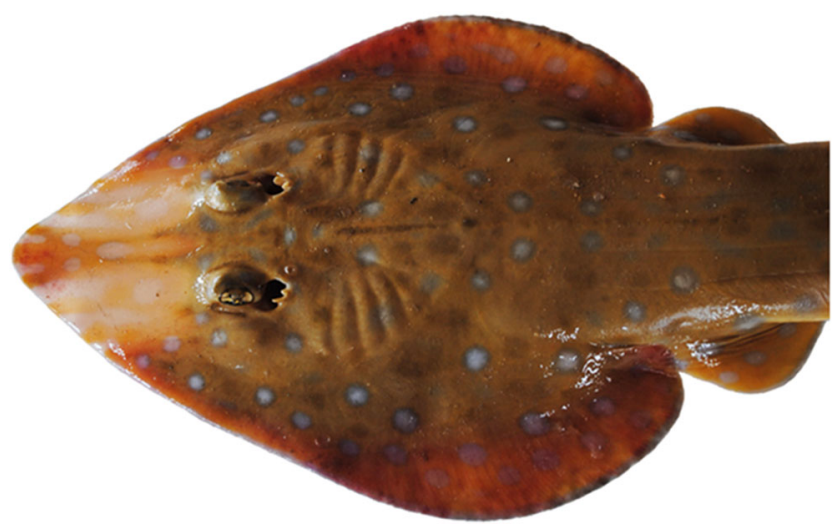

Fig. 4 Acroteriobatus andysabini sp. nov., SAIAB 97396, juvenile male holotype, $565 \mathrm{~mm}$ TL fresh, head in dorsal view taken directly after catching. Photograph by Elaine Heemstra, NRF-SAIAB

$191 \mathrm{~mm}$ TL (after preservation), juvenile male, Secteur de Fort Dauphin, southeastern Madagascar, $25^{\circ} 8.3^{\prime} \mathrm{S}, 47^{\circ} 9.1^{\prime} \mathrm{E}, 77 \mathrm{~m}$ depth, FV NOSY BE 11, station CP 3571, beam trawl, 8 May 2010; CAS-ICH 247251 (ex SAIAB 192245), $340 \mathrm{~mm}$ TL (fresh), $325 \mathrm{~mm}$ TL (after preservation), juvenile male, off southeastern Madagascar, $25^{\circ} 3.16^{\prime} \mathrm{S}, 47^{\circ} 4.41^{\prime}$ E, ACEP08352/ASCLME survey, trawl 13, collector J. Escobar, depth of capture 69 m, 8 September 2008; MNHN 1929-223, 603 mm TL, juvenile female, collected between Tuléar (Toliara) and Ankilibe, southwestern Madagascar, collector G. Petit; MNHN 1985-231, $318 \mathrm{~mm}$ TL, juvenile male, Toliara, southwestern Madagascar; MNHN 1989-553, 466 mm TL, juvenile male, Fort Dauphin (Tôlanaro), southeastern Madagascar; AMNH 232484, 265 mm TL, juvenile male, field number PD-00-XII11 MAD, off northeastern Madagascar, Antongil Bay, Ambodipaka, $16^{\circ} 4.59^{\prime} \mathrm{S}, 49^{\circ} 37^{\prime} \mathrm{E}$, local fishermen, $\mathrm{P}$.

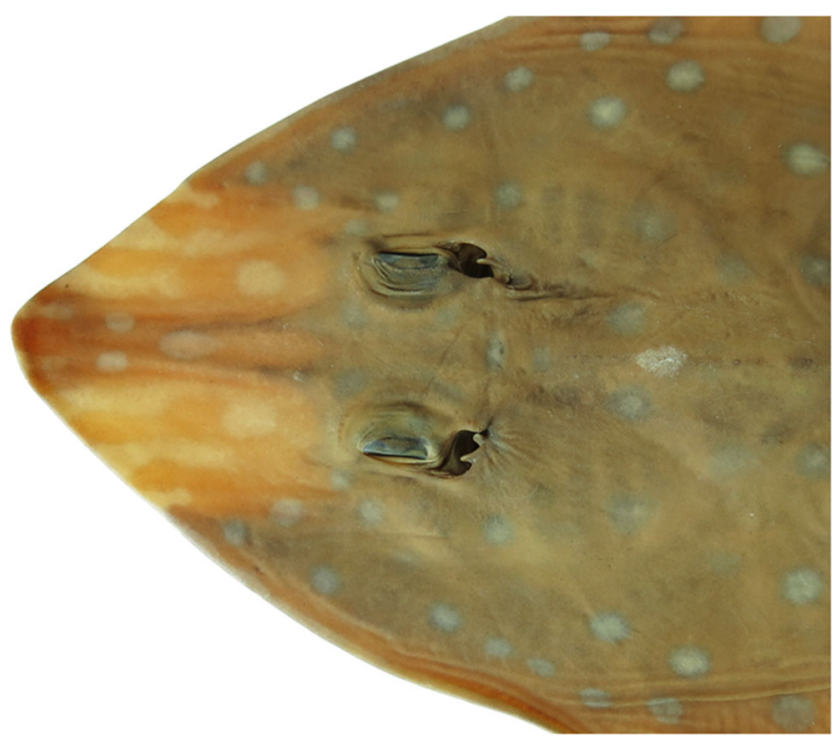

Fig. 5 Acroteriobatus andysabini sp. nov., SAIAB 97396, juvenile male holotype, $550 \mathrm{~mm}$ TL, head in dorsal view. Photograph courtesy Marsha Englebrecht (C)

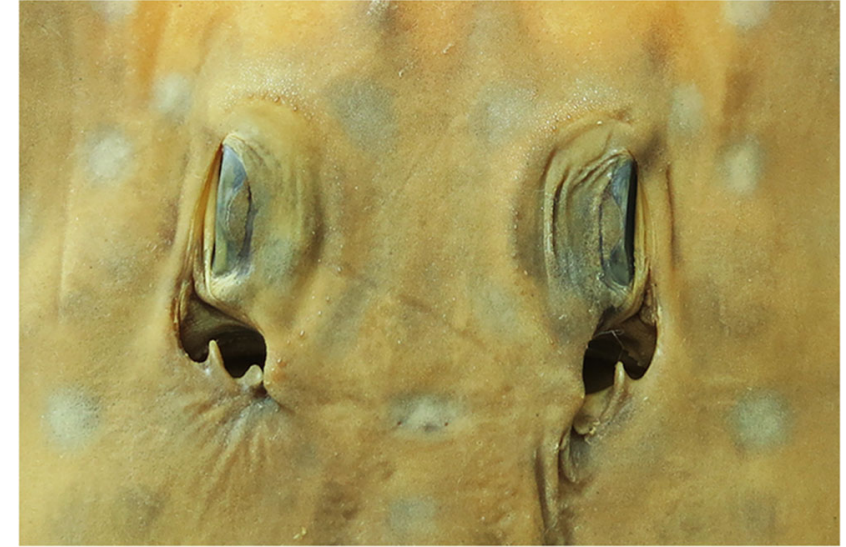

Fig. 6 Acroteriobatus andysabini sp. nov., SAIAB 97396, juvenile male holotype, $550 \mathrm{~mm} \mathrm{TL}$, close-up of orbital and spiracular region. Photograph courtesy Marsha Englebrecht $($ )

Doukakis \& Mananjo, 11 December 2000; AMNH 232511, $361 \mathrm{~mm}$ TL, juvenile female, field number PD-01-III-27 MAD, off northeastern Madagascar, Antongil Bay, Rantohely, $15^{\circ} 38.59^{\prime} \mathrm{S}, 49^{\circ} 39^{\prime} \mathrm{E}$, local fishermen, P. Doukakis, 27 March 2001; USNM 171058, 177 mm TL, male embryo, Madagascar, FV NOSY BE, collector J. Millot, March 1955.

\section{Diagnosis}

A large Acroteriobatus species distinguished by the following combination of characters: dorsal surface smooth, without prominent thorns or tubercles, except for enlarged granular denticles partially around orbital rims and irregularly distributed along midline from nape to somewhat anterior to first dorsal-fin origin; absent between dorsal fins and upper caudal fin. Snout semi-translucent with elongated bluish-gray spots giving "stripe-nosed" appearance, numerous small bluishgray spots covering disc and pectoral, pelvic, dorsal, and caudal fins on a brown background with larger, slightly darker, indistinct brown spots covering the tail and fin bases; outer edges of pelvic-fin margins white, lateral tail folds striped

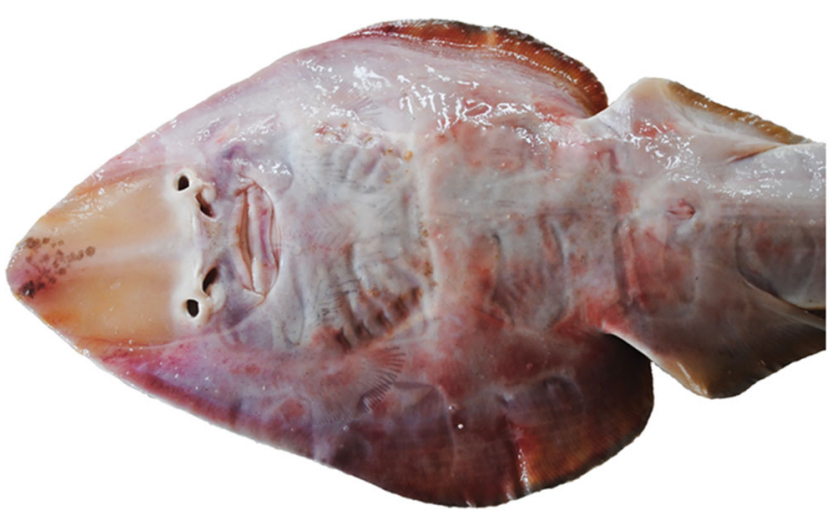

Fig. 7 Acroteriobatus andysabini sp. nov., SAIAB 97396, juvenile male holotype, $565 \mathrm{~mm}$ TL fresh, head in ventral view taken directly after catching. Photograph by Elaine Heemstra, NRF-SAIAB 


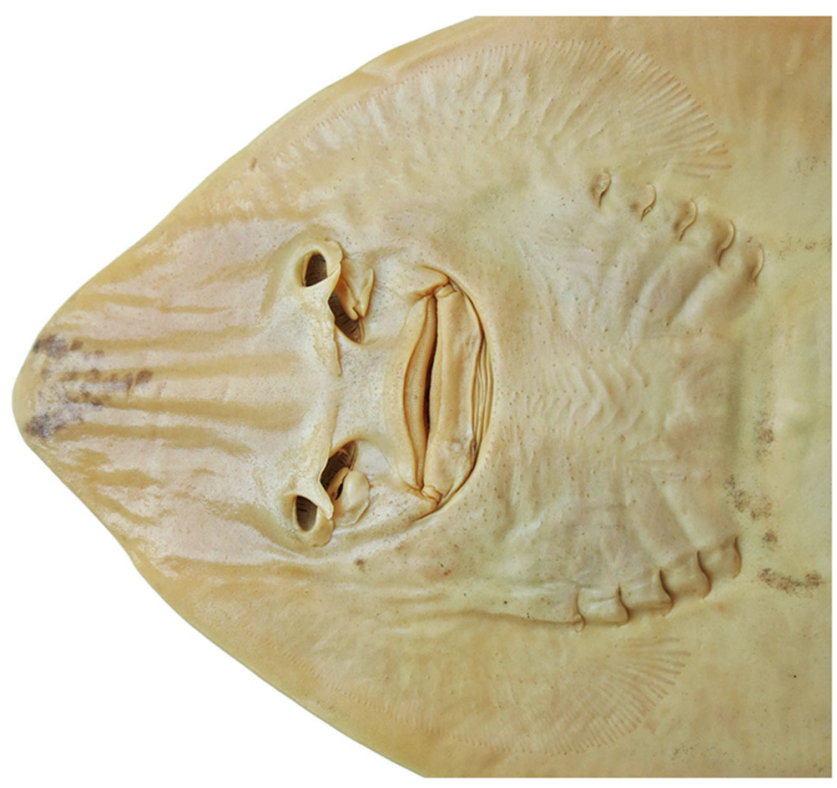

Fig. 8 Acroteriobatus andysabini sp. nov., SAIAB 97396, juvenile male holotype, $550 \mathrm{~mm}$ TL, head in ventral view. Photograph courtesy Marsha Englebrecht (C)

orange and white; ventral surface white, except for a V-shape pattern of faint to dark speckled black spots on snout tip. Nasal lamellae 42-48; upper jaw tooth row count $\sim 80-87$; 179-185 post-synarcual centra; 195-200 total vertebral segments; 6468 total pectoral skeleton radials.

\section{Description of the holotype}

Values of the eight paratypes at SAIAB and CAS, as well as the two larger paratypes at MNHN in parentheses, more complex differences are described separately. Where relevant, ratios are based on horizontal measurements unless otherwise stated. Detailed morphometric measurements and meristics of the holotype, eight paratypes at SAIAB and CAS, and the two larger paratypes at MNHN are given in Table 1.

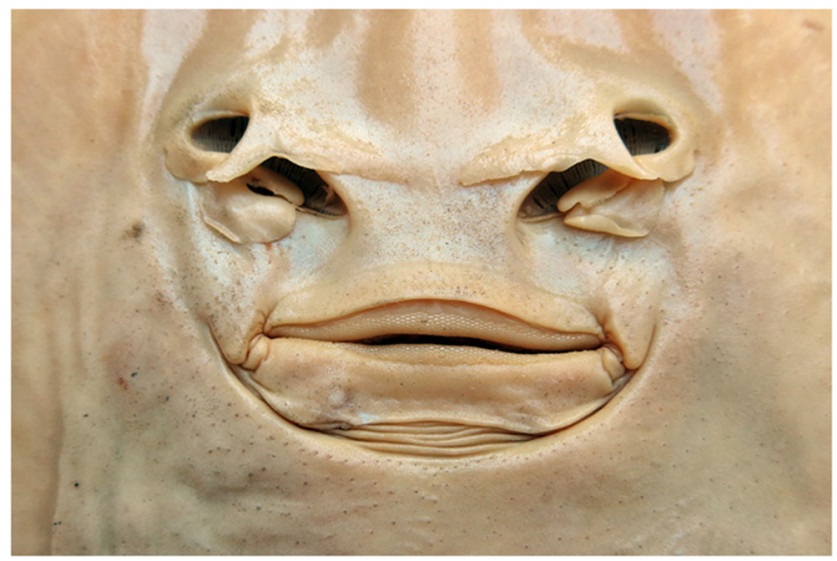

Fig. 9 Acroteriobatus andysabini sp. nov., SAIAB 97396, juvenile male holotype, $550 \mathrm{~mm}$ TL, close-up of mouth-nasal region. Photograph courtesy Marsha Englebrecht (C)

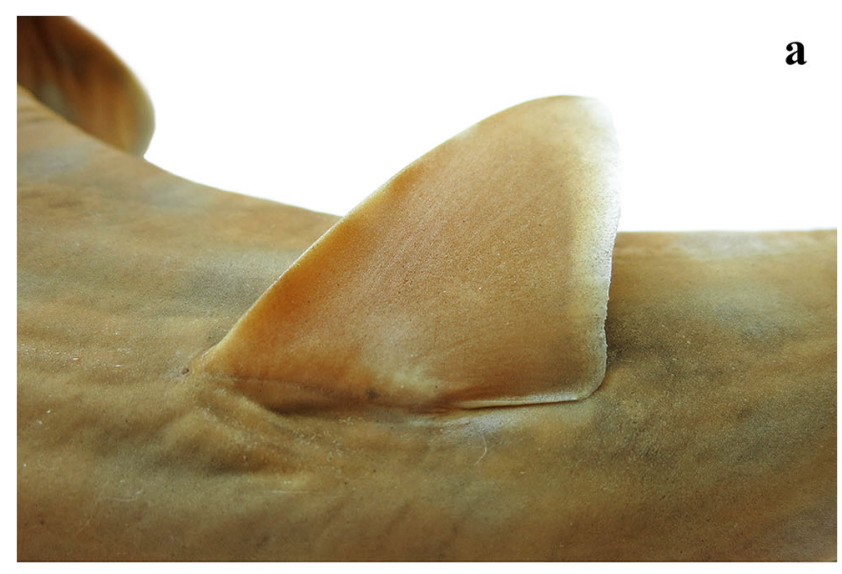

b

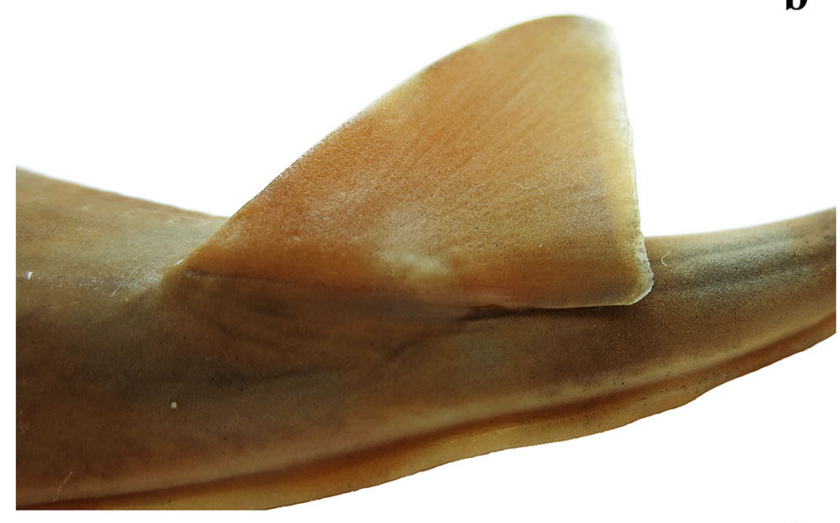

C

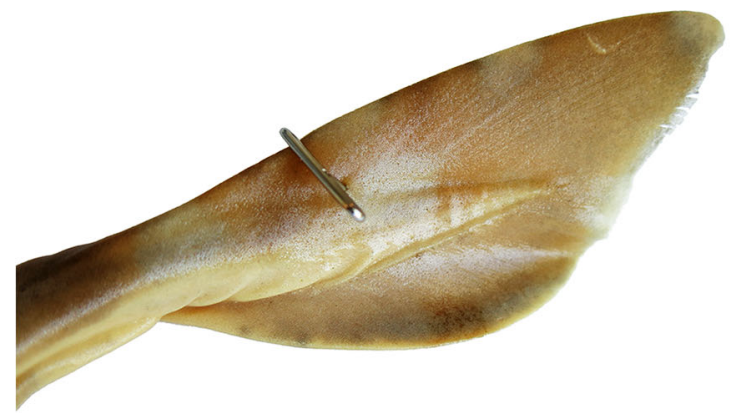

Fig. 10 Acroteriobatus andysabini sp. nov., SAIAB 97396, juvenile male holotype, $550 \mathrm{~mm}$ TL; first dorsal (a), second dorsal (b), and caudal (c) fins in lateral views (c image reversed). Photographs courtesy Marsha Englebrecht (C)

External morphology (Figs. 1, 2, 3, 4, 5, 6, 7, 8, 9, and 10) Disc wedge-shaped, narrowly angular anteriorly, snout angle in front of eyes $79.1^{\circ}\left(76-84.6^{\circ}\right)$; anterior margin undulate, outer pectoral margin broadly rounded, posterior margin convex, rear tip broadly rounded; disc length $1.22(1.13-1.24)$ times its width. Pelvic fins elongate, short-based, base length 1.82 (0.82-1.76) times inner margin length; pelvic-fin total length 1.55 (1.43-2.13) times base length, 2.29 (1.95-3.96) times width; anterior margin weakly concave anteriorly, becoming 


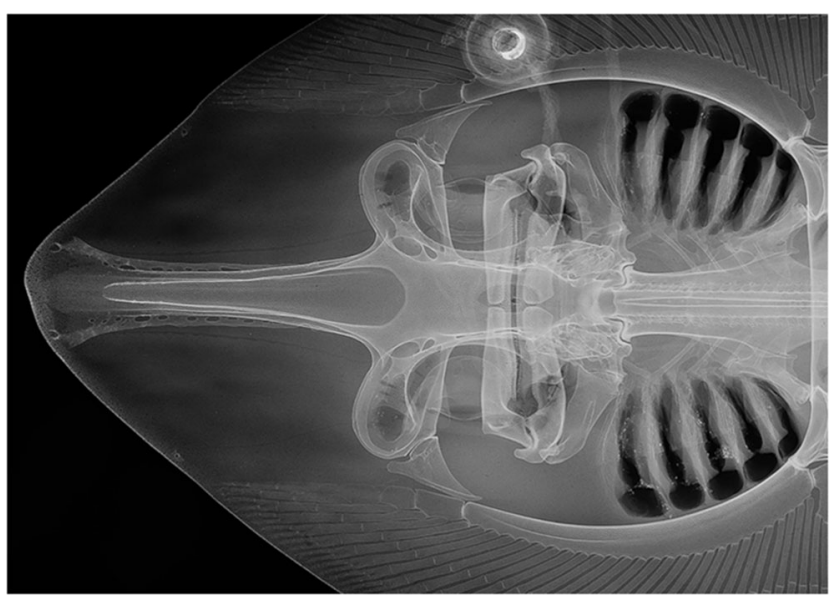

Fig. 11 Acroteriobatus andysabini sp. nov., SAIAB 97396, juvenile male holotype, $550 \mathrm{~mm}$ TL, radiograph of cranium and snout in dorsal view. The radiograph was taken and kindly provided by Jon Fong

convex posterior to constriction at the level of first propterygials, turning to weakly concave posterior to the level of posterior margin of spiracles and again becoming weakly convex towards broadly rounded apex, posterior margin weakly convex, free rear tip narrowly rounded. Tail elongate, becoming slender, tapering weakly to caudal; depressed, in cross section rounded above, nearly flat below; tail length from anterior cloaca 1.47 (1.29-1.81) times precloacal length, $1.43(1.25-1.64)$ times disc length, and $5.95(4.28-6.42)$ times body width at pelvic-fin insertions; body width 2.2 (2.253.22) times depth at pelvic-fin insertions, $2.41(2.5-3.17)$ at first dorsal-fin origin, $2.23(1.58-2.5)$ at second dorsal-fin origin. Dermal fold lateral along tail, its origin just posterior to pelvic-fin insertion, extending to just behind the ventral origin of caudal-fin; fold well developed, maximum width at interdorsal space slightly more than the width of posterior nasal flap (Figs. 1, 2, and 3).

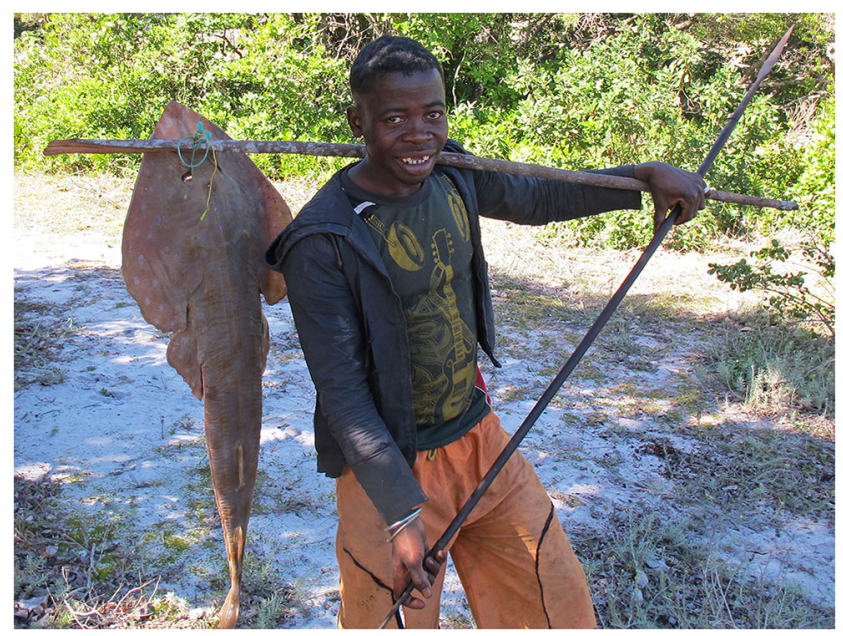

Fig. 12 Acroteriobatus andysabini sp. nov., Malagasy artisanal fisherman with large ( $\sim 1000 \mathrm{~mm}$ TL) specimen. The photograph was taken and kindly provided by Kerry Sink
Head moderately elongate, ventral length 25.3 (25.2$29.8) \%$ TL, dorsal length 24.2 (25.7-33.5)\% TL; snout rather short, bluntly pointed, preoral length $2.44(2.00-2.62)$ times mouth width, 4.88 (3.75-4.89) times internarial distance, 1.15 (1.05-2.27) times dorsal caudal-fin margin, 2.86 (2.27-2.9) times distance from nostril to margin of disc; direct preorbital snout length 2.38 (1.92-2.39) times interspiracular distance, 4.06 (2.9-4.09) times orbit diameter, 3.63 (3.09-3.63) times skeletal interorbital space; interorbital space weakly concave, relatively broad; eyes moderately large, slightly elevated, but not protruding, orbit diameter $1.7(0.92-2.5)$ times spiracle length, 0.89 (0.85-1.13) times skeletal interorbital distance. Spiracles relatively large, narrowly bean-shaped; two prominent and strongly compressed folds on upper posterior margin, length of inner spiracular fold $0.7(0.5-0.7)$ times that of outer fold; distance between bases of folds $0.5(0.3-0.7)$ times length of shortest fold (Figs. 4, 5, and 6).

Nostril moderate large, oblique, all nasal flaps well developed; anterior aperture subcircular; nostril length 3.14 (2.63.83) times anterior aperture width, $1.22(0.95-1.6)$ times anterior nasal-flap base length, $0.76(0.53-0.77)$ times distance from nostril to edge of disc, 1.29 (1.0-1.28) times internarial distance. Anterior nasal-flap base greatly extended, penetrating horizontally into internarial space, mesial edges barely separated (by about width of posterior nasal flap); innermost extension of flap not narrow; flap base length 1.80 (1.6-2.6) times its width at process, 2.57 (1.67-3.28) times anterior aperture width; minimum distance between mesial insertions of flaps $7.29(4.8-13.0)$ in greatest distance across nostrils anteriorly, 2.43 (1.6-4.5) in minimum internarial distance; process of flap two or more times as long as wide, narrowing distally to blunt pointed tip, and adjoining with posterolateral nasal flap and anterior aperture posterior margin. Posterolateral nasal flap well developed, lobe-like, broadest medially, length 3.0 (1.22-3.5) times its width; origin slightly posterior to lateral extremity of anterior nasal aperture, with free fold posteriorly extending nearly to the level of anterior nasal flap insertion. Posterior nasal flap well developed, lobelike, base length 1.86 (1.67-3.0) times its width, its inner edge nearly reaching innermost margin of nostril; width 1.0 (1.0 1.67) times anterior aperture width, $1.4(1.0-2.0)$ times posterolateral nasal-flap width (Figs. 7, 8, and 9).

Mouth width 1.55 (1.44-2.0) times nostril length, 6.56 (5.13-6.71) in precloacal length; positioned beneath posterior margin of orbit. Upper jaw slightly convex, upper lip broadly arched; lower lip not pronounced, not separated from oral groove by ridges of strongly corrugated skin; corners of mouth with several strong, short lateral grooves. Teeth arranged in quincunx, small, close-set, rhombic, broad-based with short, bluntly rounded cusp; upper and lower teeth similar in shape and size; $84(\sim 80-87)$ tooth rows in upper and $\sim 79$ ( $80-87)$ in lower jaws. Gill openings strongly wavy; length of third gill slit 2.75 (1.93-3.0) in nostril length, 5.5 
Fig. 13 Map of the southwestern Indian Ocean depicting the catch locations of the examined specimens of all three species of the Acroteriobatus leucospilus species complex. Holotype (white star) and paratypes (white circles) of $A$. andysabini sp. nov., holotype (gray star) and paratypes (gray star and gray circle) of A. stehmanni sp. nov., and syntypes (black triangles) and other specimens (black squares) of A. leucospilus

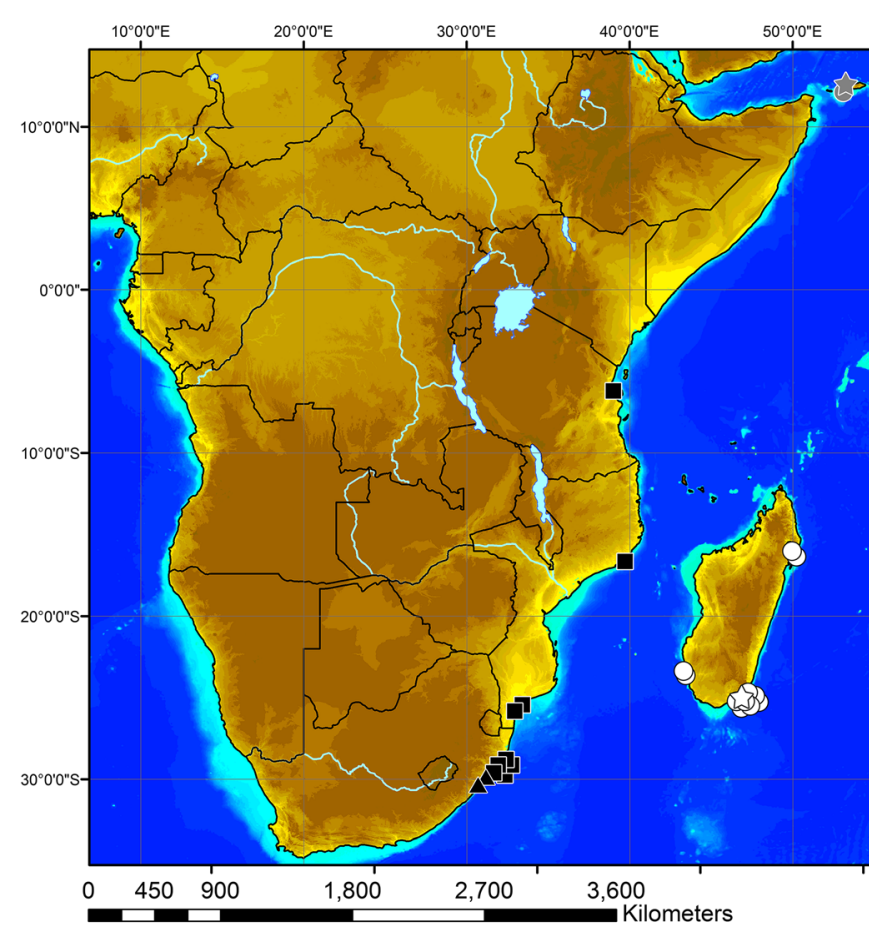

$60^{\circ} 0^{\circ} 0$ "E

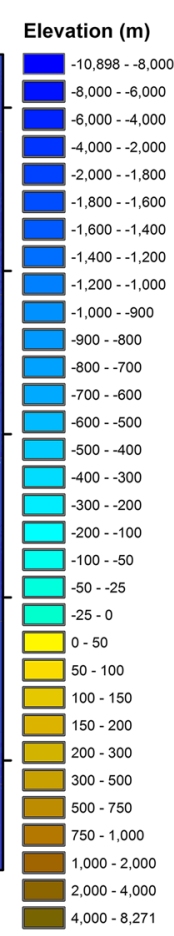

(4.75-6.67) in distance between fifth gill slits; distance between first gill slits $1.48(1.3-1.58)$ times distance between fifth gill slits; distance between fifth gill slits 2.59 (2.222.73) times internarial distance, 1.29 (1.12-1.54) times mouth width, $0.32(0.29-0.37)$ of ventral head length (Fig. 9).

Dorsal fins erect, relatively tall, dissimilar in size and shape; first dorsal-fin anterior margin weakly convex, curving rearwards towards rounded apex; posterior margin weakly convex near tip, then becoming weakly concave to nearly straight; second dorsal-fin anterior margin weakly convex, curving rearwards towards pointed apex; posterior margin nearly straight; free rear tips rounded of both dorsal fins, almost forming right angle, not produced; first dorsal fin height subequal to second, length of first $0.93(0.92-1.44)$ times height, its base length 2.15 (1.17-2.5) times inner margin length; second dorsal-fin length $1.0(0.93-1.42)$ times its height, base length 1.47 (1.34-2.21) times inner margin length (Fig. 10a, b). First dorsal-fin origin well posterior to pelvic-fin free rear tips, interspace $1.29(1.0-1.15)$ times interdorsal distance; interdorsal space relatively short, 1.48 (1.16-2.09) times second dorsal-fin height, $2.11(1.88-2.88)$ times base of first dorsal fin, $1.11(0.71-1.25)$ times tail width at origin of first dorsal fin, 1.59 (1.11-1.9) times interspace between second dorsal-fin insertion and upper origin of caudal fin. Caudal fin relatively small; dorsal caudal margin weakly convex, length 1.6 (1.53-2.08) times preventral margin length; ventral lobe broadly rounded; posterior margin weakly concave; fin tip acutely pointed (Fig. 10c).

Dermal denticles mostly minute, close-set, covering entire body and fins; surfaces mostly smooth to the touch, except for a continuous row of $\sim 15$ to 20 somewhat enlarged granular and smaller denticles around the anterior and inner rim of orbits, extending to the anterior edge of inner spiracle; enlarged, coarse denticles patchily distributed along midline from nape to $\sim 50 \mathrm{~mm}$ anterior to first dorsal-fin origin, absent along midline between dorsal fins and upper caudal origin; enlarged denticles absent on snout tip, anterior and lateral margins of disc, pelvic fins, and bases of dorsal fins; nasal flaps and lamellae naked. Crowns of denticles mostly oval, except largest denticles with slightly erect, bluntly acute cusp, base stellate. Thorns absent. Prebranchial sensory pore patch distinct, extending to just behind the level of third gill slit. Post-scapular sensory canal rather indistinct, weakly undulated anteriorly, terminating near pectoral-fin insertions; sensory pores minute, canal not forming a shallow groove (Fig. 3).

Rostral cartilage broad, its shaft just slightly increasing in width posteriorly from rostral node; rostral node rounded at apex, not angular, broadly expanded, relatively short, axis at maximum node width 34.1 (33.6-39.3)\% of length of rostral cartilage from tip; anterior fontanelle relatively broad posteriorly, tapering gradually towards rostral node, dorsolateral edges of cartilage surrounding fontanelle (rostral ridges on surface of snout) well separated posteriorly, not constricting medially; rostral cartilage 57.5 (52.6-57.1)\% of neurocranium length, ventral edges of rostral cartilage united; nasal capsules large, with their transverse axes directed anterolaterally; width of cranium across nasal capsules 1.25 (1.17-1.27) times nasobasal length (base of rostrum to occipital condyles); width of nasal capsule $1.79(1.56-1.87)$ times its length; basal plate relatively broad, its minimum width $3.37(3.15-3.75)$ times in 
Table 1 Acroteriobatus andysabini sp. nov., morphometrics and meristics

\begin{tabular}{|c|c|c|c|c|c|c|c|c|}
\hline & \multicolumn{2}{|c|}{$\begin{array}{l}\text { Acroteriobatus } \\
\text { andysabini sp. } \\
\text { nov., juvenile } \\
\text { male holotype, } \\
\text { SAIAB } 97396\end{array}$} & \multicolumn{2}{|c|}{$\begin{array}{l}\text { Acroteriobatus } \\
\text { andysabini sp. } \\
\text { nov., juvenile } \\
\text { female paratype, } \\
\text { SAIAB } 189815\end{array}$} & \multirow{2}{*}{$\begin{array}{l}\begin{array}{l}\text { Minimum } \\
(n=9)\end{array} \\
\% \mathrm{TL}\end{array}$} & \multirow{2}{*}{$\begin{array}{l}\begin{array}{l}\text { Maximum } \\
(n=9)\end{array} \\
\% \mathrm{TL}\end{array}$} & \multirow{2}{*}{$\begin{array}{l}\begin{array}{l}\text { Mean } \\
(n=11)\end{array} \\
\% \mathrm{TL}\end{array}$} & \multirow[t]{2}{*}{$\mathrm{SD}$} \\
\hline & $\mathrm{mm}$ & $\% \mathrm{TL}$ & $\mathrm{mm}$ & $\% \mathrm{TL}$ & & & & \\
\hline Total length (mm) & 550.0 & 100.0 & 345.0 & 100.0 & 178.0 & 603.0 & 351.2 & \\
\hline Disc width, max & 187.0 & 34.0 & 117.0 & 33.9 & 32.8 & 39.3 & 35.7 & 2.4 \\
\hline Disc length, direct/point-to-point (ptp) & 228.0 & 41.5 & 145.0 & 42.0 & 38.1 & 45.1 & 42.3 & 2.2 \\
\hline Head length, dorsal (to occipital joint) & 133.0 & 24.2 & 98.0 & 28.4 & 25.7 & 33.5 & 29.0 & 2.9 \\
\hline Head length, ventral (to 5th gill slit), ptp & 139.0 & 25.3 & 93.0 & 27.0 & 25.0 & 29.8 & 27.3 & 1.8 \\
\hline Snout length, presocket & 69.0 & 12.5 & 44.0 & 12.8 & 11.4 & 15.2 & 13.4 & 1.2 \\
\hline Orbit diameter, ptp & 17.0 & 3.1 & 12.0 & 3.5 & 3.2 & 5.2 & 4.0 & 0.7 \\
\hline Spiracle length & 10.0 & 1.8 & 7.0 & 2.0 & 1.8 & 3.7 & 2.4 & 0.6 \\
\hline Orbit and spiracle length & 27.0 & 4.9 & 18.0 & 5.2 & 4.7 & 7.4 & 6.0 & 1.1 \\
\hline Interorbital width 1 (skeletal) & 19.0 & 3.5 & 13.0 & 3.8 & 3.7 & 4.7 & 4.0 & 0.4 \\
\hline Interspiracular width & 29.0 & 5.3 & 19.0 & 5.5 & 4.8 & 7.3 & 6.0 & 0.9 \\
\hline Preoral length & 83.0 & 15.1 & 53.0 & 15.4 & 14.3 & 17.8 & 16.0 & 1.3 \\
\hline MOW, mouth width & 34.0 & 6.2 & 21.0 & 6.1 & 6.0 & 8.4 & 6.8 & 0.9 \\
\hline Prenarial length, ptp & 67.0 & 12.2 & 42.0 & 12.2 & 11.3 & 14.1 & 12.6 & 1.0 \\
\hline NOW, nostril length & 22.0 & 4.0 & 13.0 & 3.8 & 3.0 & 4.7 & 4.0 & 0.5 \\
\hline AAW, anterior nasal aperture, width & 7.0 & 1.3 & 5.0 & 1.4 & 1.0 & 1.7 & 1.3 & 0.2 \\
\hline ANF, anterior nasal flap, base length & 18.0 & 3.3 & 12.0 & 3.5 & 2.8 & 4.1 & 3.4 & 0.5 \\
\hline ANW, anterior nasal flap, width & 10.0 & 1.8 & 7.0 & 2.0 & 1.4 & 2.5 & 1.8 & 0.3 \\
\hline PLT, posterolateral nasal flap, total length & 10.0 & 1.8 & 8.0 & 2.3 & 1.6 & 3.6 & 2.5 & 0.6 \\
\hline PLW, posterolateral nasal flap, width & 5.0 & 0.9 & 3.0 & 0.9 & 0.8 & 1.1 & 1.0 & 0.1 \\
\hline PNF, posterior nasal flap, base length & 13.0 & 2.4 & 8.0 & 2.3 & 1.8 & 3.1 & 2.4 & 0.4 \\
\hline PNW, posterior nasal flap, width & 7.0 & 1.3 & 3.0 & 0.9 & 1.0 & 1.4 & 1.1 & 0.2 \\
\hline INM, distance between lateral margins of anterior apertures & 51.0 & 9.3 & 31.0 & 9.0 & 8.3 & 12.6 & 10.3 & 1.5 \\
\hline INW, distance between posterior margins of nostrils & 17.0 & 3.1 & 11.0 & 3.2 & 3.0 & 4.5 & 3.7 & 0.6 \\
\hline INA, distance between insertions of anterior nasal flaps & 7.0 & 1.3 & 5.0 & 1.4 & 0.7 & 2.6 & 1.3 & 0.6 \\
\hline Shortest direct distance from anterior opening of nostril to disc margin & 29.0 & 5.3 & 21.0 & 6.1 & 4.9 & 7.7 & 6.1 & 0.9 \\
\hline Gill opening width, 3rd & 8.0 & 1.5 & 5.0 & 1.4 & 1.4 & 2.2 & 1.7 & 0.3 \\
\hline Distance between first gill openings & 65.0 & 11.8 & 43.0 & 12.5 & 11.4 & 14.6 & 12.9 & 1.2 \\
\hline Distance between fifth gill openings & 44.0 & 8.0 & 29.0 & 8.4 & 7.3 & 10.7 & 9.1 & 1.2 \\
\hline Pelvic fin, length & 96.0 & 17.5 & 59.0 & 17.1 & 15.2 & 16.6 & 16.3 & 0.6 \\
\hline Pelvic fin, anterior margin length & 40.0 & 7.3 & 22.0 & 6.4 & 4.6 & 7.6 & 6.6 & 0.9 \\
\hline Pelvic fin, width & 42.0 & 7.6 & 25.0 & 7.2 & 4.2 & 7.9 & 7.2 & 1.0 \\
\hline Pelvic fin, base length & 62.0 & 11.3 & 34.0 & 9.9 & 7.2 & 11.4 & 9.9 & 1.2 \\
\hline Pelvic fin, inner margin length & 34.0 & 6.2 & 28.0 & 8.1 & 6.2 & 8.7 & 7.2 & 0.9 \\
\hline First dorsal fin, length & 39.0 & 7.1 & 23.0 & 6.7 & 5.9 & 7.1 & 6.7 & 0.4 \\
\hline First dorsal fin, anterior margin length & 53.0 & 9.6 & 28.0 & 8.1 & 8.0 & 10.1 & 9.0 & 0.7 \\
\hline First dorsal fin, height & 42.0 & 7.6 & 23.0 & 6.7 & 4.6 & 7.4 & 6.6 & 0.9 \\
\hline First dorsal fin, base length & 28.0 & 5.1 & 15.0 & 4.3 & 3.8 & 4.7 & 4.3 & 0.4 \\
\hline First dorsal fin, inner margin length & 13.0 & 2.4 & 6.0 & 1.7 & 2.3 & 3.4 & 2.7 & 0.5 \\
\hline Second dorsal fin, length & 40.0 & 7.3 & 25.0 & 7.2 & 6.1 & 7.9 & 7.2 & 0.5 \\
\hline Second dorsal fin, anterior margin length & 52.0 & 9.5 & 29.0 & 8.4 & 8.5 & 9.8 & 9.1 & 0.4 \\
\hline Second dorsal fin, height & 40.0 & 7.3 & 21.0 & 6.1 & 5.5 & 7.9 & 6.5 & 0.8 \\
\hline Second dorsal fin, base length & 25.0 & 4.5 & 17.0 & 4.9 & 3.9 & 5.2 & 4.7 & 0.4 \\
\hline Second dorsal fin, inner margin length & 17.0 & 3.1 & 8.0 & 2.3 & 2.0 & 3.4 & 2.8 & 0.5 \\
\hline
\end{tabular}


Table 1 (continued)

\begin{tabular}{|c|c|c|c|c|c|c|c|c|}
\hline & \multicolumn{2}{|c|}{$\begin{array}{l}\text { Acroteriobatus } \\
\text { andysabini sp. } \\
\text { nov., juvenile } \\
\text { male holotype, } \\
\text { SAIAB } 97396\end{array}$} & \multicolumn{2}{|c|}{$\begin{array}{l}\text { Acroteriobatus } \\
\text { andysabini sp. } \\
\text { nov., juvenile } \\
\text { female paratype, } \\
\text { SAIAB } 189815\end{array}$} & \multirow{2}{*}{$\begin{array}{l}\text { Minimum } \\
(n=9)\end{array}$} & \multirow{2}{*}{$\begin{array}{l}\text { Maximum } \\
(n=9)\end{array}$} & \multirow{2}{*}{$\begin{array}{l}\begin{array}{l}\text { Mean } \\
(n=11)\end{array} \\
\% \mathrm{TL}\end{array}$} & \multirow[t]{2}{*}{ SD } \\
\hline & $\mathrm{mm}$ & $\% \mathrm{TL}$ & $\mathrm{mm}$ & $\% \mathrm{TL}$ & & & & \\
\hline Caudal fin, dorsal margin & 72.0 & 13.1 & 50.0 & 14.5 & 13.1 & 15.4 & 14.3 & 0.7 \\
\hline Caudal fin, preventral margin & 45.0 & 8.2 & 29.0 & 8.4 & 6.3 & 9.5 & 8.0 & 1.1 \\
\hline Snout to pelvic-fin origin ptp & 198.0 & 36.0 & 132.0 & 38.3 & 34.5 & 40.8 & 38.1 & 2.0 \\
\hline Snout to anterior vent & 223.0 & 40.5 & 141.0 & 40.9 & 35.6 & 43.6 & 40.9 & 2.5 \\
\hline Snout to first dorsal-fin origin & 330.0 & 60.0 & 205.0 & 59.4 & 58.4 & 61.5 & 59.7 & 0.9 \\
\hline Snout to second dorsal-fin origin & 413.0 & 75.1 & 257.0 & 74.5 & 73.5 & 76.3 & 74.4 & 0.9 \\
\hline Snout to upper caudal-fin origin & 472.0 & 85.8 & 295.0 & 85.5 & 83.1 & 86.7 & 85.2 & 1.1 \\
\hline Snout to lower caudal-fin origin & 470.0 & 85.5 & 303.0 & 87.8 & 84.6 & 88.2 & 86.8 & 1.1 \\
\hline Pelvic-fin insertion to dorsal-fin origin & 76.0 & 13.8 & 42.0 & 12.2 & 9.6 & 13.9 & 11.8 & 1.5 \\
\hline Interdorsal distance & 59.0 & 10.7 & 38.0 & 11.0 & 7.1 & 11.6 & 10.2 & 1.3 \\
\hline Disc width, anterior orbit & 114.0 & 20.7 & 75.0 & 21.7 & 19.1 & 26.2 & 22.8 & 2.4 \\
\hline Body width, pelvic insertion & 55.0 & 10.0 & 38.0 & 11.0 & 9.0 & 14.1 & 11.1 & 1.6 \\
\hline Body width, first dorsal-fin origin & 53.0 & 9.6 & 35.0 & 10.1 & 8.4 & 10.8 & 9.5 & 0.8 \\
\hline Body width, second dorsal-fin origin & 29.0 & 5.3 & 19.0 & 5.5 & 4.3 & 5.5 & 4.9 & 0.5 \\
\hline Body depth, maximum & 29.0 & 5.3 & 18.0 & 5.2 & 4.5 & 5.7 & 5.1 & 0.3 \\
\hline Body depth, pelvic-fin insertion & 25.0 & 4.5 & 15.0 & 4.3 & 3.4 & 5.0 & 4.3 & 0.5 \\
\hline Body depth, first dorsal-fin origin & 22.0 & 4.0 & 13.0 & 3.8 & 3.0 & 4.1 & 3.6 & 0.4 \\
\hline Body depth, second dorsal-fin origin & 13.0 & 2.4 & 8.0 & 2.3 & 2.0 & 2.7 & 2.3 & 0.2 \\
\hline Caudal peduncle length $=$ dorsal $(\mathrm{D} 2)$ - caudal space & 37.0 & 6.7 & 21.0 & 6.1 & 5.1 & 8.4 & 6.8 & 0.9 \\
\hline Tail length from anterior vent & 327.0 & 59.5 & 204.0 & 59.1 & 56.4 & 64.4 & 59.1 & 2.5 \\
\hline Angle before eyes, ${ }^{\circ}$ & \multicolumn{2}{|c|}{79.1} & \multicolumn{2}{|c|}{80.9} & 76.0 & 84.6 & 80.5 & 2.4 \\
\hline
\end{tabular}

Individual values for the juvenile male holotype (SAIAB 97396) and one female paratype (SAIAB 189815), ranges for all other SAIAB and CAS as well as the two larger MNHN paratypes $(n=9)$, and means and standard deviations (SD) for all nine SAIAB and CAS as well as the two larger MNHN type specimens are presented. Proportional values are expressed as percentages of total length (TL) $70 \%$ ethanol preserved except for minimum, maximum, and mean of TL in $\mathrm{mm}$

nasobasal length; cranial roof with small, oval-shaped fenestra, with its anterior edge located behind precerebral cavity by a distance $\sim 1.5$ times its length; anterior cartilage triangular, narrow, posteriorly wedge-shaped, without an anterior lobe extending past nasal capsules; preorbital processes well developed; postorbital processes moderately large, not bifurcate; greatest width across processes $1.91(1.79-1.99)$ times in nasobasal length (Fig. 11).

Nasal lamellae 45 (42-48). Pectoral skeleton with 32 (3032 ) propterygial, $9(8-10)$ mesopterygial, 2 (1-2) neopterygial, 25 (24-25) metapterygial, amounting to 68 (64-68) total radials. Total pelvic-fin radials 25 (24-27). Total vertebral segment (synarcual and free) counts 196 (195-200); post-synarcual centra 180 (179-185); precaudal centra (excluding synarcual centra) 139 (137-144); synarcual segments 16 (14-17); monospondylous precaudal centra 39 (37-42); diplosondylous precaudal centra 100 (98-104); diplosondylous caudal centra 41 (40-42). Monospondylous to diplosondylous centra transition posterior to pelvic girdle.

\section{Coloration}

Prior to preservation: dorsal surface light to medium brown with an orangish tinge, an organized pattern of smaller bluishgray circular spots, with a darker ring encircling a lighter bluish-gray center, and indistinct larger brownish spots (Figs. 1 and 4); smaller bluish-gray spots cover the snout, disc, pectoral, pelvic, dorsal, and caudal fins; larger brownish spots mostly cover the tail and fin bases, but not extending onto fins; snout semi-translucent with somewhat elongated bluish-gray spots giving it a stripe-nosed appearance (Figs. 1, 4, and 12). Outer edges of pelvic-fin margins white, outer edges of posterior dorsal-fin margins translucent whitish, lateral tail folds striped orange and white. Ventral surface mostly white, except 


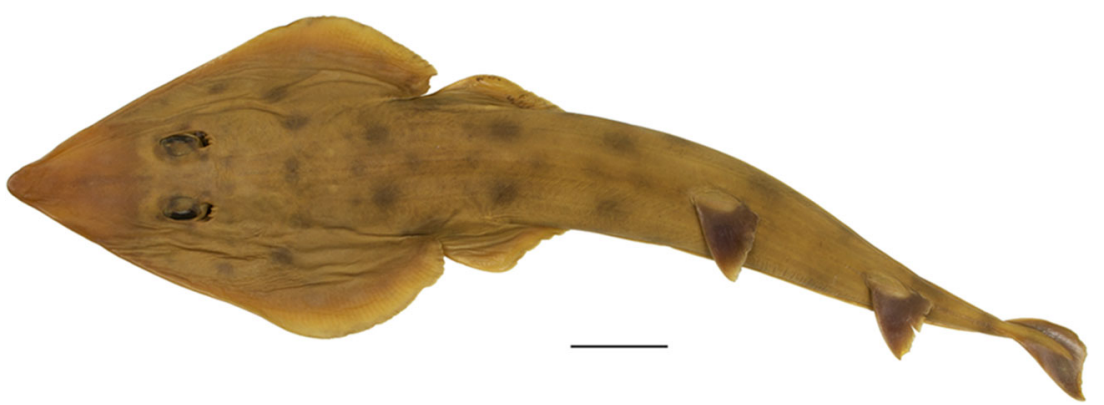

Fig. 14 Acroteriobatus stehmanni sp. nov., ZMH 25553, adult male holotype, $597 \mathrm{~mm}$ TL, in total dorsal view. Scale bar: $5 \mathrm{~cm}$

for faint to dark speckled black spots forming a V-shape on rostral snout tip; spots becoming darker and more numerous with growth (Fig. 7); in small ( $\leq 215 \mathrm{~mm}$ TL), newborn specimens short $\mathrm{V}$-shaped black lines with faint dark spots at base extending from tip to about one-third snout length to midpoint of internostril space; larger individuals ( $>300 \mathrm{~mm} \mathrm{TL}$ ) with prominent dark to black spots, becoming more numerous and with spots extending to about the midpoint of rostrum; pectoral and pelvic fins mostly white, except fin edges brownish (Fig. 7). After preservation: dorsal surface similar but bluish-gray spots less pronounced and large brownish spots mostly faded and not evident (Fig. 2). Ventral surface also similar, except edges of pectoral and pelvic-fin margins fade to a lighter brown (Fig. 3).

\section{Size}

The new species is described from 15 specimens (ten males and five females), ranging in size from 177 to $603 \mathrm{~mm}$ TL. All types are juvenile, the size at birth is $\sim 180 \mathrm{~mm}$ TL. Based on images of unretained specimens (Fig. 12), Acroteriobatus andysabini sp. nov. is a large species of Acroteriobatus growing to more than $1000 \mathrm{~mm}$ TL.

\section{Distribution}

Acroteriobatus andysabini sp. nov. is presently only known from the coastal waters surrounding Madagascar and may be endemic to this region (Fig. 13). Records are from off Tôlanaro, Anosy region, and Cape Andavaka (southeastern Madagascar), Toliara and Ankilibe (southwestern
Madagascar), and Antongil Bay (northeastern Madagascar). Its depth range is from shallow coastal waters to at least 80 m. Fricke et al. (2018) reported A. leucospilus as a new species record for Madagascar but commented that the species had been misidentified previously as Rhinobatos (= Acroteriobatus) annuulatus by several earlier authors.

\section{Etymology}

The species name andysabini is in honor of Andy Sabin for his gracious support of the Lost Sharks project at the Pacific Shark Research Center.

Acroteriobatus stehmanni sp. nov. Weigmann, Ebert \& Séret http://zoobank.org/8C6C09A5-4110-4ADF-A879-024A31EC8E3A (Socotra blue-spotted guitarfish)

(Figs. 14, 15, 16, 17, 18, 19, 20, 21, 22, 23, 24, 25, 26, and 27, Table 2)

Acroteriobatus leucospilus—Manilo \& Bogorodsky (2003: S93)?

The holotype and seven paratypes are deposited in the Zoological Museum Hamburg (ZMH), and two paratypes are deposited in the National Museum of Natural History, National Academy of Sciences of Ukraine (NASU), Kyiv (Kiev) (HMNH NASU).

Holotype ZMH 25553, adult male, $597 \mathrm{~mm}$ TL fresh, $597 \mathrm{~mm}$ TL $70 \%$ ethanol preserved, off Socotra Islands, $12^{\circ} 39^{\prime} \mathrm{N}, 53^{\circ} 27^{\prime} \mathrm{E}-12^{\circ} 36^{\prime} \mathrm{N}, 53^{\circ} 20^{\prime} 2^{\prime \prime} \mathrm{E}, 41-43$ m depth, RV Vityaz, cruise 17, station $2567,30 \mathrm{~m}$ bottom trawl, trawl \# 7, on the bottom from 8:30 to 9:27 p.m., 28 October 1988, collected by Matthias F.W. Stehmann.

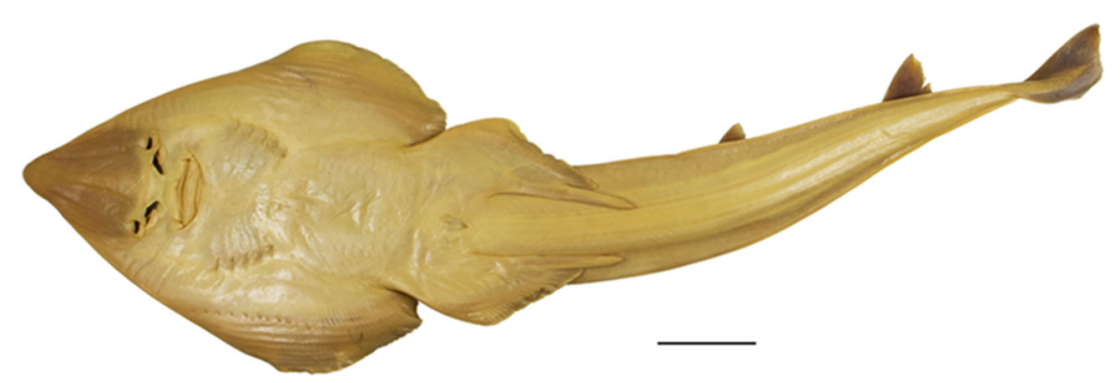

Fig. 15 Acroteriobatus stehmanni sp. nov., ZMH 25553, adult male holotype, $597 \mathrm{~mm}$ TL, in total ventral view. Scale bar: $5 \mathrm{~cm}$ 


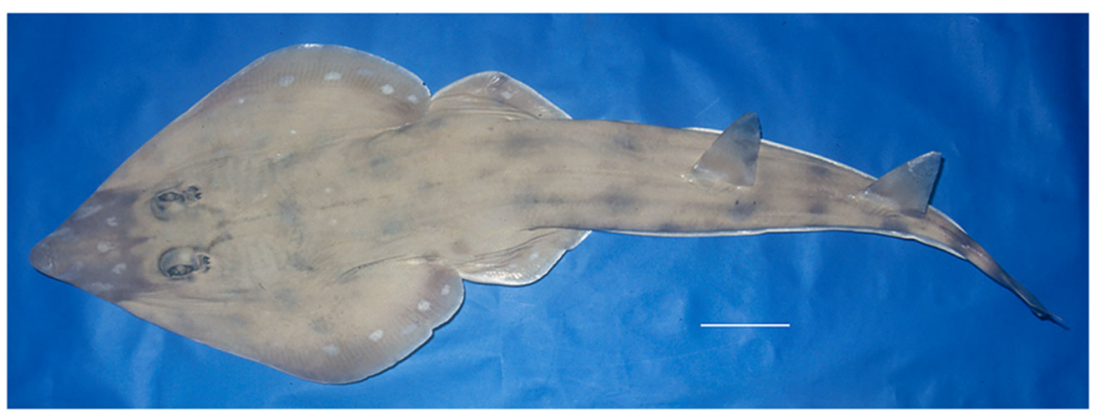

Fig. 16 Acroteriobatus stehmanni sp. nov., ZMH 25557, adult female paratype, $602 \mathrm{~mm}$ TL fresh, in total dorsal view taken directly after catching. Scale bar: $5 \mathrm{~cm}$. The photograph was taken and kindly provided by Matthias Stehmann

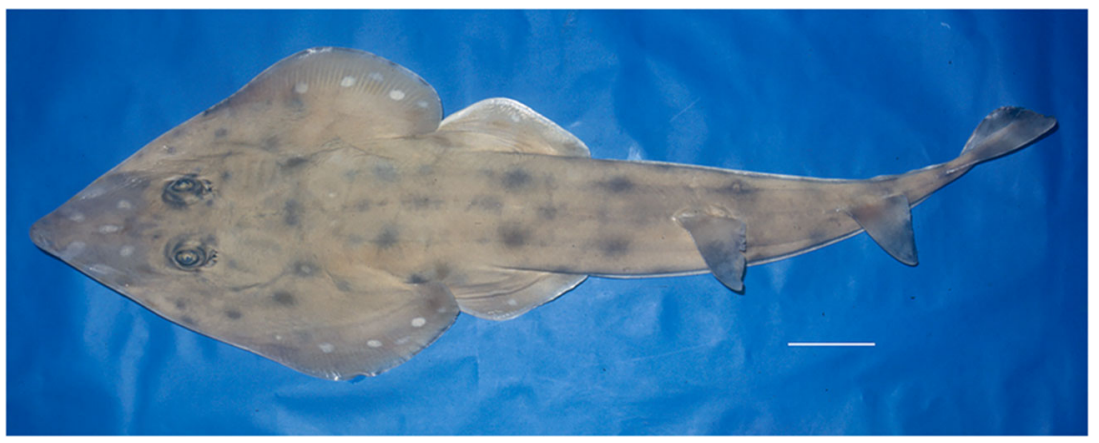

Fig. 17 Acroteriobatus stehmanni sp. nov., ZMH 25558, adult female paratype, $594 \mathrm{~mm}$ TL fresh, in total dorsal view taken directly after catching. Scale bar: $5 \mathrm{~cm}$. The photograph was taken and kindly provided by Matthias Stehmann

Paratypes (9) ZMH 25554, adult male, $564 \mathrm{~mm}$ TL fresh, $562 \mathrm{~mm}$ TL $70 \%$ ethanol preserved, data the same as holotype ZMH 25553; ZMH 25555, adult male, $546 \mathrm{~mm}$ TL fresh, $547 \mathrm{~mm}$ TL $70 \%$ ethanol preserved, data the same as holotype ZMH 25553; ZMH 25556, very early subadult male, $378 \mathrm{~mm}$ TL fresh, $376 \mathrm{~mm}$ TL 70\% ethanol preserved, data the same as holotype ZMH 25553; ZMH 25557, adult female, $602 \mathrm{~mm}$ TL fresh, $603 \mathrm{~mm}$ TL $70 \%$ ethanol preserved, data the same as holotype ZMH 25553; ZMH 25558, adult female, $594 \mathrm{~mm}$ TL fresh, $591 \mathrm{~mm}$ TL 70\% ethanol preserved, data the same as holotype ZMH 25553; ZMH 25559, adult male, $622 \mathrm{~mm}$ TL fresh, $613 \mathrm{~mm}$ TL $70 \%$ ethanol preserved, off Socotra Islands, $12^{\circ} 04^{\prime} 48^{\prime \prime} \mathrm{N}, 53^{\circ} 12^{\prime} 36^{\prime \prime} \mathrm{E}-12^{\circ} 09^{\prime} 12^{\prime \prime} \mathrm{N}, 53^{\circ} 10^{\prime} 6^{\prime \prime} \mathrm{E}, 36-40 \mathrm{~m}$ depth, RV Vityaz, cruise 17, station 2829, $29 \mathrm{~m}$ shrimp trawl, trawl \# 100, on the bottom from 10:23 to 11:30 p.m., 15 January 1989, collected by Matthias F.W. Stehmann; ZMH 25560, juvenile male, $208 \mathrm{~mm}$ TL fresh, $201.4 \mathrm{~mm}$ TL 70\% ethanol preserved, data the same as paratype ZMH 25559; HMNH NASU 103836, adult male, $567 \mathrm{~mm}$ TL fresh, $568 \mathrm{~mm}$ TL $4 \%$ formaldehyde preserved, data the same as holotype ZMH 25553; HMNH NASU 103838, female, caudal-tip missing, $523 \mathrm{~mm}$ TL fresh, $518 \mathrm{~mm}$ TL $4 \%$ formaldehyde preserved, data the same as holotype ZMH 25553.

\section{Diagnosis}

A small Acroteriobatus species distinguished by the following combination of characters: dorsal surface smooth, without prominent thorns or tubercles, except for slightly enlarged granular denticles partially around orbital rims and rather regularly distributed along midline from nape to or to somewhat anterior to first dorsal-fin origin; absent between dorsal fins

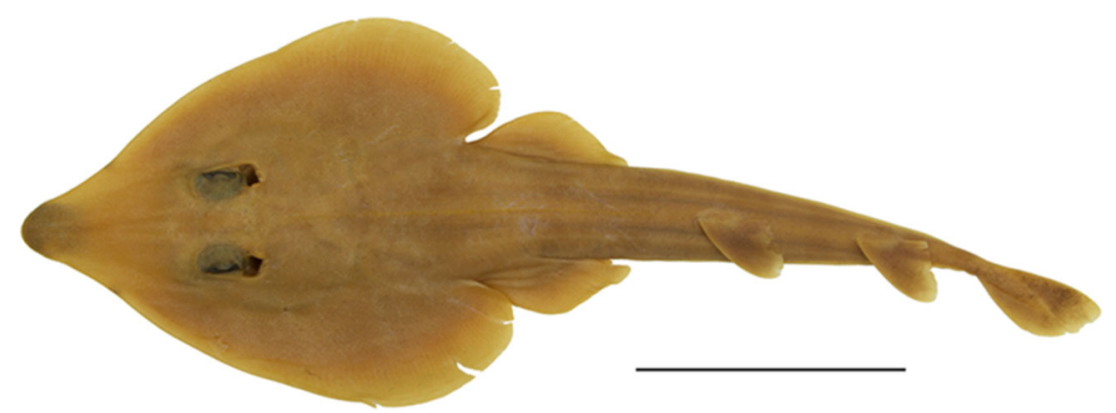

Fig. 18 Acroteriobatus stehmanni sp. nov., ZMH 25560, juvenile male paratype, $201.4 \mathrm{~mm}$ TL, in total dorsal view. Scale bar: $5 \mathrm{~cm}$ 


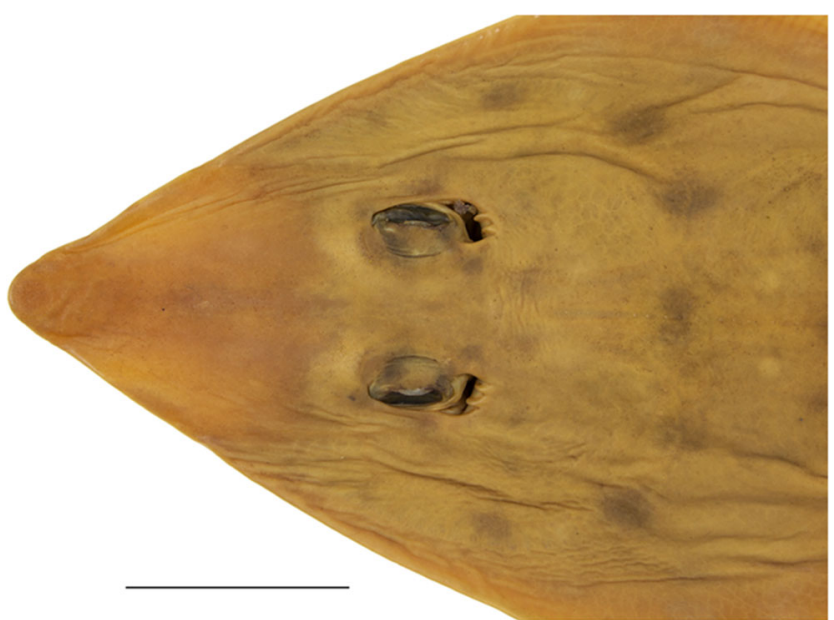

Fig. 19 Acroteriobatus stehmanni sp. nov., ZMH 25553, adult male holotype, $597 \mathrm{~mm}$ TL, head in dorsal view. Scale bar: $5 \mathrm{~cm}$

and upper caudal fin. Snout semi-translucent with a few, somewhat elongated bluish-gray spots only giving it a very reduced stripe-nosed appearance, patterning with small bluish-gray circular spots generally sparse and confined to symmetrical patterns on snout tip, posterior pectoral-fin margins, a pair of tiny spots on midbody behind occipital joint, and few spots on posterior pelvic-fin margins; indistinct brown spots on the body and dorsal and caudal fins; ventral surface white except for a blackish blotch and two tiny black spots on ventral snout tip in smallest juvenile paratype. Nasal lamellae 43-48; upper jaw tooth row count $\sim 64-78 ; 181-186$ post-synarcual centra; 194-200 total vertebral segments; 6367 total pectoral skeleton radials.

\section{Description of the holotype}

Values of the seven ZMH paratypes in parentheses; more complex differences are described separately. Where relevant,

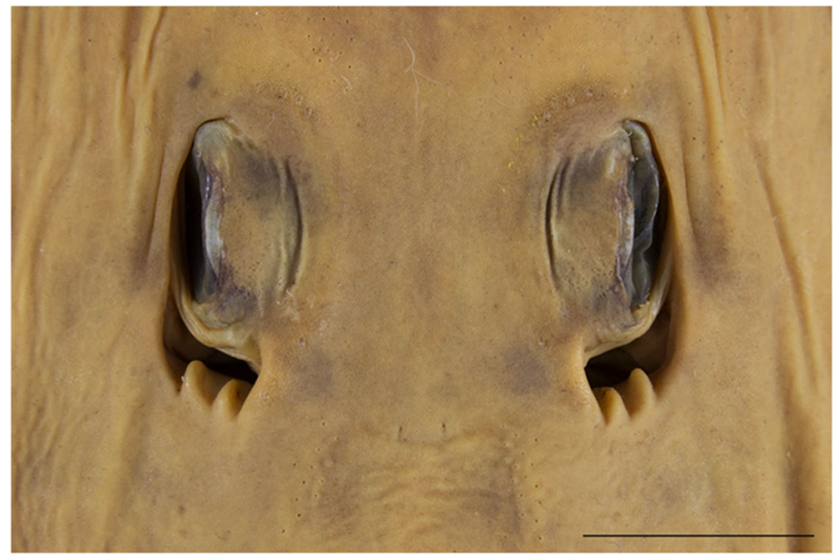

Fig. 20 Acroteriobatus stehmanni sp. nov., ZMH 25553, adult male holotype, $597 \mathrm{~mm}$ TL, close-up of orbital and spiracular region. Scale bar: $2 \mathrm{~cm}$

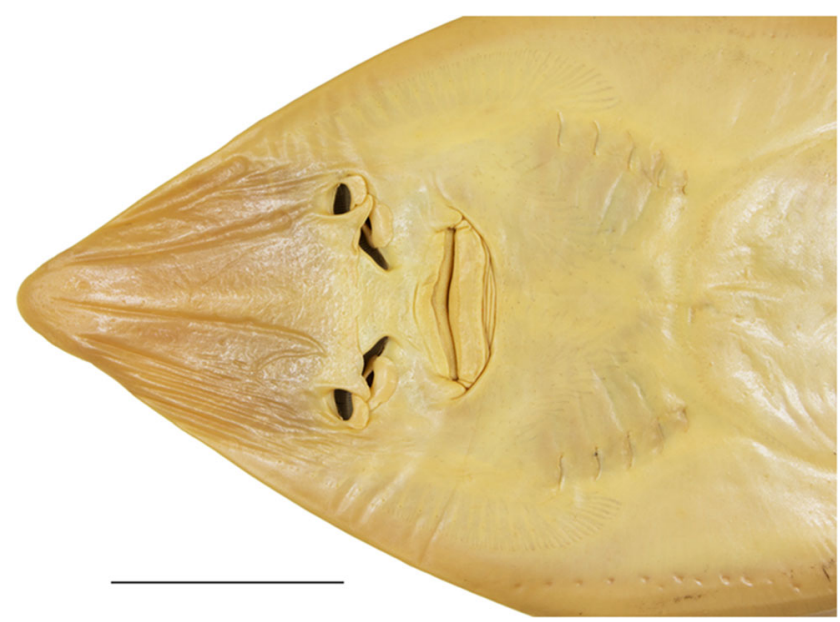

Fig. 21 Acroteriobatus stehmanni sp. nov., ZMH 25553, adult male holotype, $597 \mathrm{~mm}$ TL, head in ventral view. Scale bar: $5 \mathrm{~cm}$

ratios are based on horizontal measurements unless otherwise stated. Detailed morphometric measurements and meristics of the holotype and seven ZMH paratypes are given in Table 2.

External morphology (Figs. 14, 15, 16, 17, 18, 19, 20, 21, 22, $23,24,25$, and 27) Disc wedge-shaped, narrowly angular anteriorly, snout angle before eyes moderately acute, $72^{\circ}(71-$ $77^{\circ}$ ); anterior margin straight, outer pectoral margin broadly rounded, posterior margin convex, rear tip broadly rounded; disc length $1.3(1.25-1.32)$ times its width. Pelvic fins elongate, short-based, base length $1.0(0.81-1.47)$ times inner margin length; pelvic-fin total length $1.86(1.56-2.18)$ times base length, 2.93 (2.52-3.34) times width; anterior margin straight, becoming weakly convex towards broadly rounded apex, posterior margin weakly convex, free rear tip narrowly rounded. Tail elongate, slightly constricted in anterior part between

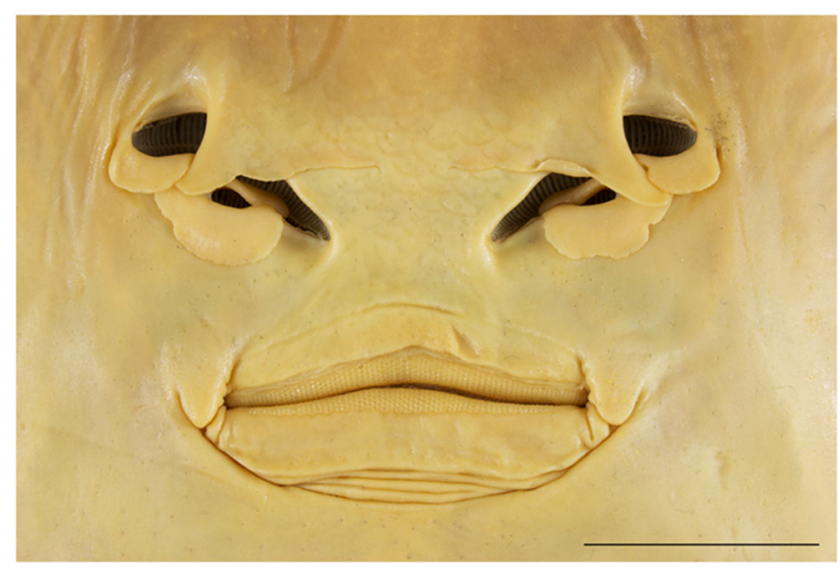

Fig. 22 Acroteriobatus stehmanni sp. nov., ZMH 25553, adult male holotype, $597 \mathrm{~mm}$ TL, close-up of mouth-nasal region. Scale bar: $2 \mathrm{~cm}$ 

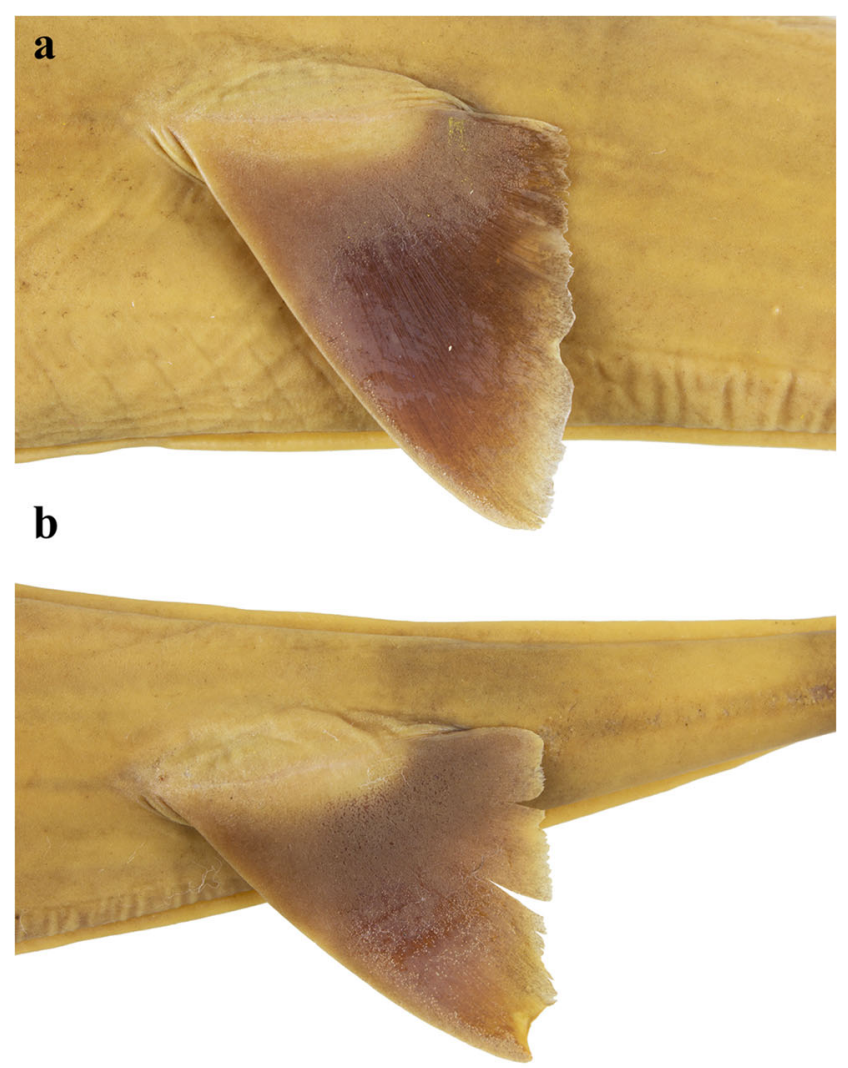

C

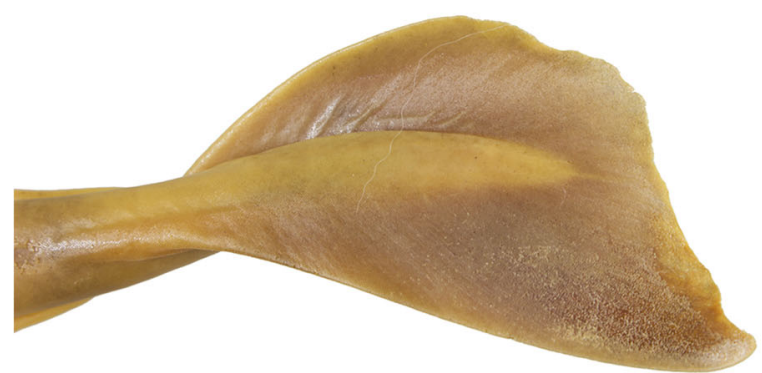

Fig. 23 Acroteriobatus stehmanni sp. nov., ZMH 25553, adult male holotype, $597 \mathrm{~mm}$ TL; first dorsal (a), second dorsal (b), and caudal (c) fins in lateral views. Scale bar: $2 \mathrm{~cm}$

pelvic-fin origin and about level pelvic-fin free rear tip, broadest somewhat anterior to first dorsal-fin origin, tapering posteriorly; very strongly depressed dorsoventrally, in cross section nearly flat below, more rounded above; tail length from anterior cloaca $1.61(1.29-1.69)$ times precloacal length, 1.62 (1.25-1.69) times disc length, and 6.34 (5.41-6.82) times body width at pelvic-fin insertions; body width 2.29 (2.092.46) times depth at pelvic-fin insertions, $2.63(2.57-2.91)$ at first dorsal-fin origin, $2.18(2.08-2.27)$ at second dorsal-fin origin. Dermal fold lateral along tail, originating about level pelvic-fin free rear tip and reaching onto base of caudal fin just behind ventral origin of caudal fin; fold well developed, maximum width at interdorsal space more than half width of posterior nasal flap and more than length of longest spiracular fold (Figs. 14, 15, 16, 17, and 18).

Head relatively short, direct ventral length 24.4 (23.6-25.3 except for 29.7 in smallest juvenile paratype)\% TL, dorsal length 17.8 (17.2-22.8)\% TL; snout rather short, bluntly pointed, preoral length 2.94 (2.63-3.02) times mouth width, 6.9 (5.46-6.75) times internarial distance, 1.32 (1.26-1.38) times dorsal caudal-fin margin, 4.66 (3.37-4.5) times distance from nostril to margin of disc; direct preorbital snout length 2.78 (2.59-2.72) times interspiracular distance, 3.76 (3.4$3.73)$ times orbit diameter, $4.32(3.83-4.23)$ times skeletal interorbital space; interorbital space weakly concave, rather narrow; eyes moderately large, slightly elevated, but not protruding, orbit diameter $1.8(1.68-2.26)$ times spiracle length, $1.15(1.03-1.23)$ times skeletal interorbital space. Spiracles relatively large, narrowly bean-shaped; two prominent and strongly compressed folds on upper posterior margin, length of inner spiracular fold $0.73(0.59-0.75)$ times that of outer fold; distance between bases of folds 0.85 (0.95-1.83) times length of shortest fold (Figs. 19 and 20).

Nostril moderately large, oblique, all nasal flaps well developed; anterior aperture subcircular; nostril length 3.35 (2.67-2.98) times anterior aperture width, $1.16(0.91-1.15)$ times anterior nasal-flap base length, $1.01(0.6-0.93)$ times distance from nostril to edge of disc, $1.49(0.98-1.39)$ times internarial distance. Anterior nasal-flap base greatly extended, penetrating horizontally into internarial space, mesial edges barely separated (by about width of posterior nasal flap); innermost extension of flap not narrow; flap base length 1.87 (1.82-2.19) times its width at process, 2.9 (2.47-3.21) times anterior aperture width; minimum distance between mesial insertions of flaps $8.24(7.39-10.4)$ in greatest distance across nostrils anteriorly, 2.29 (2.56-3.17) in minimum internarial distance; process of flap about twice as long as wide, narrowing distally to blunt pointed tip, and adjoining with posterolateral nasal flap and anterior aperture posterior margin. Posterolateral nasal flap well developed, lobe-like, broadest medially, length $3.9(2.3-4.29)$ times its width; originating just behind lateral extremity of anterior nasal aperture, extending posteromesially as a free fold almost to the level of insertion of posterior nasal flap. Posterior nasal flap well developed, lobe-like, base length $2.16(1.49-2.46)$ times its width, its inner edge nearly reaching innermost margin of nostril; width $0.88(0.65-1.11)$ times anterior aperture width, $1.26(0.88-1.48)$ times posterolateral nasal-flap width (Figs. 21 and 22).

Mouth width 1.57 (1.56-1.95) times nostril length, 7.11 (6.51-7.53) in precloacal length; positioned beneath posterior margin of orbit. Upper jaw slightly convex, upper lip broadly arched; lower lip not pronounced, not separated from oral groove by ridges of strongly corrugated skin; corners of mouth with several strong, short lateral grooves. Teeth 
Fig. 24 Acroteriobatus stehmanni sp. nov., enlarged dermal denticles of a-e adult male holotype, ZMH 25553, $597 \mathrm{~mm}$ $\mathrm{TL}$, and $\mathbf{f}-\mathbf{i}$ smallest juvenile paratype, ZMH 25560, male, $201.4 \mathrm{~mm}$ TL. a, b preorbital denticles in a overview and $\mathbf{b}$ close-up view; c postorbital denticle; $\mathbf{d}$ spiracular denticles; $\mathbf{e}$ midline denticles; $\mathbf{f}$ preorbital denticles; g postorbital denticles; h spiracular denticle; i midline denticles. Scale bars: a $2 \mathrm{~mm}$ and b-i $1 \mathrm{~mm}$

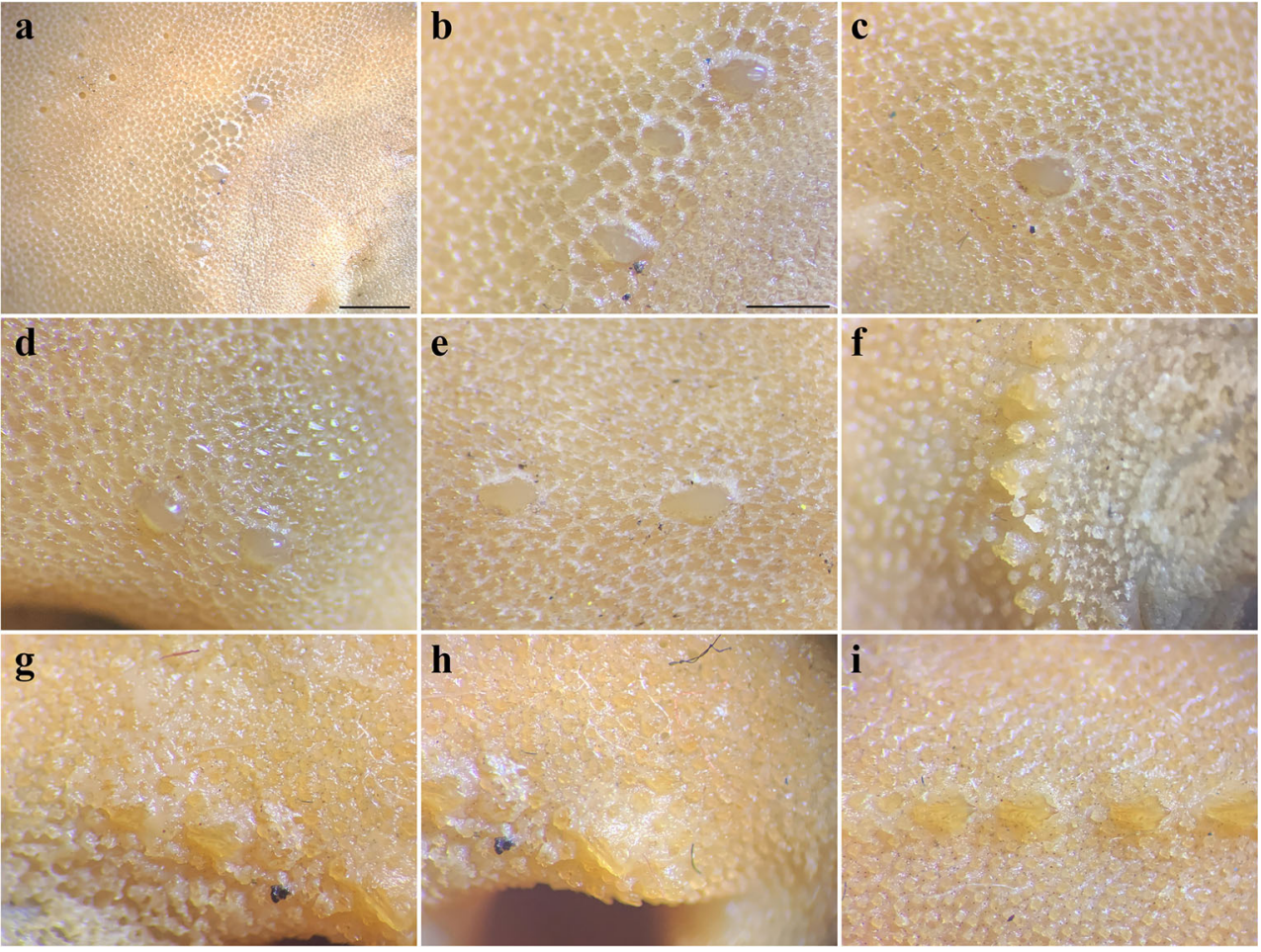

arranged in quincunx, small, close-set, rhombic, broad-based with short, bluntly rounded cusp; upper and lower teeth similar in shape and size; $72(\sim 64-78)$ tooth rows in upper and $\sim 70$ ( $\sim 64-76)$ in lower jaws. Gill openings strongly wavy; length of third gill slit 2.68 (2.08-2.73) in nostril length, 6.00 (5.92-6.45) in distance between fifth gill slits; distance between first gill slits $1.42(1.39-1.48)$ times distance between fifth gill slits; distance between fifth gill slits 3.34 (2.79-3.53)
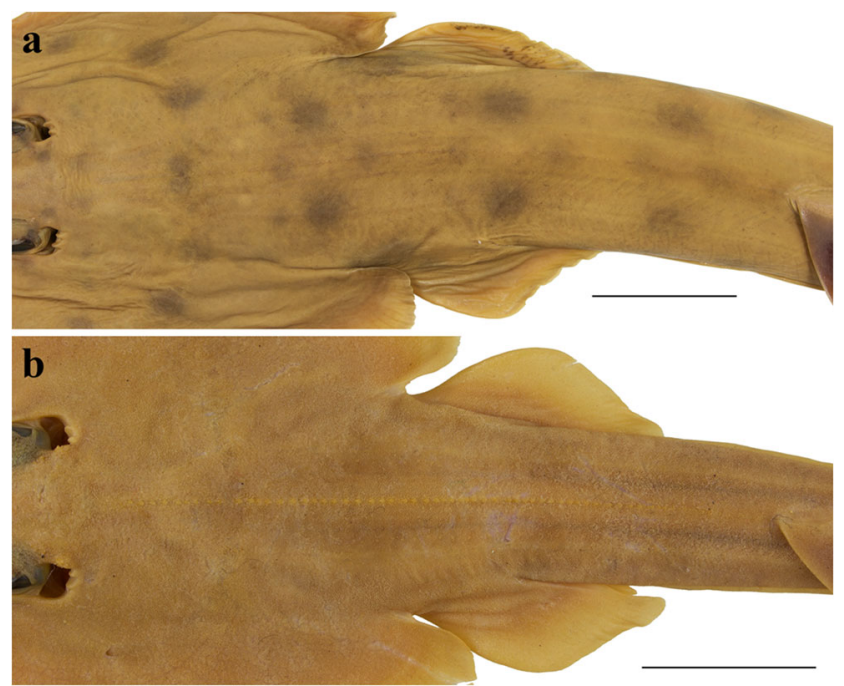

Fig. 25 Acroteriobatus stehmanni sp. nov., row of enlarged dermal denticles along midline from nape to first dorsal-fin origin of a adult male holotype, ZMH 25553, $597 \mathrm{~mm}$ TL, and b smallest juvenile paratype, ZMH 25560, male, $201.4 \mathrm{~mm}$ TL. Scale bars: a $5 \mathrm{~cm}$ and b $2 \mathrm{~cm}$ times internarial distance, 1.43 (1.4-1.6) times mouth width, 0.32 (0.31-0.36) of ventral head length (Fig. 22).

Dorsal fins erect, relatively tall, dissimilar in size and shape; first dorsal-fin anterior margin weakly convex, curving rearwards towards pointed (pointed to rounded) apex; posterior margin weakly convex near tip, then becoming weakly concave to nearly straight; second dorsal-fin anterior margin weakly convex, curving towards pointed apex; posterior margin nearly straight; free rear tips rounded of both dorsal fins, almost forming right angle, not produced; first dorsal fin height subequal to second, length of first $1.0(0.91-1.15)$ times height, its base length 2.76 (1.61-2.86) times inner margin length; second dorsal-fin length $1.12(0.96-1.19)$ times its

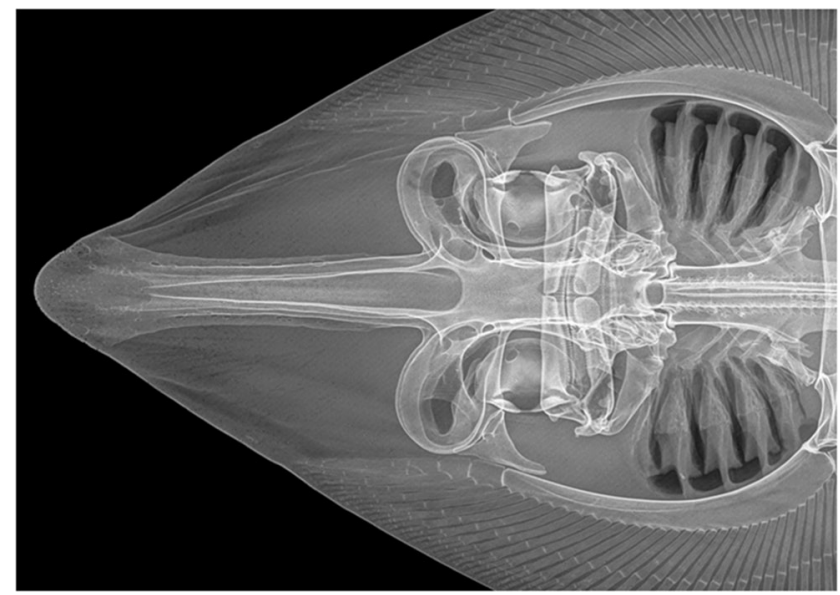

Fig. 26 Acroteriobatus stehmanni sp. nov., ZMH 25553, adult male holotype, $597 \mathrm{~mm}$ TL, radiograph of cranium and snout in dorsal view 


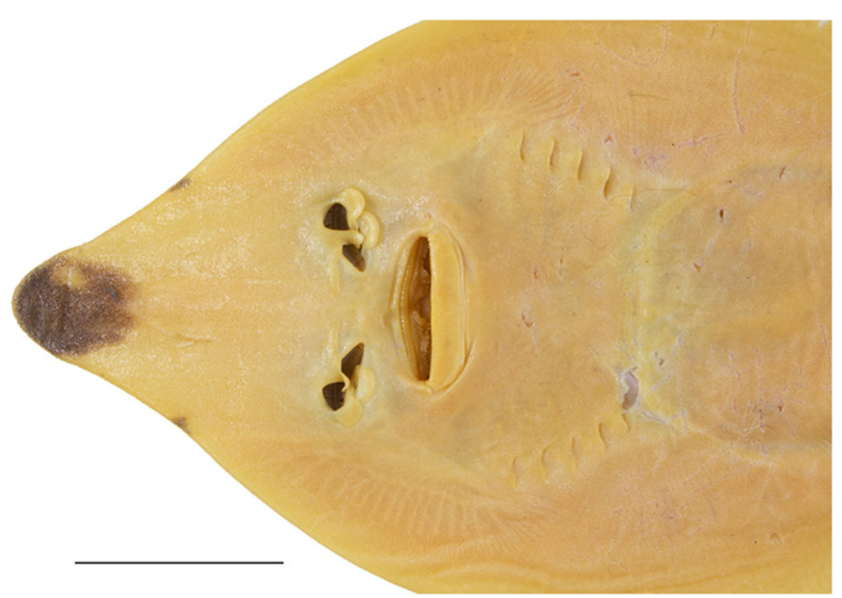

Fig. 27 Acroteriobatus stehmanni sp. nov., ZMH 25560, juvenile male paratype, $201.4 \mathrm{~mm}$ TL, head in ventral view. Scale bar: $2 \mathrm{~cm}$

height, base length 3.05 (2.01-3.5) times inner margin length (Fig. 23a, b). First dorsal-fin origin well posterior to pelvic-fin free rear tips, interspace $1.29(1.2-1.3)$ times interdorsal distance; interdorsal space relatively short, 2.05 (1.61-2.17) times second dorsal-fin height, 2.54 (2.3-2.82) times base of first dorsal fin, $1.34(1.08-1.44)$ times tail width at origin of first dorsal fin, 1.85 (1.71-2.05) times interspace between second dorsal-fin insertion and upper origin of caudal fin. Caudal fin relatively small; dorsal caudal margin weakly convex, length 1.5 (1.45-1.73) times preventral margin length; ventral lobe broadly rounded; posterior margin weakly concave; fin tip acutely pointed (Fig. 23c).

Dermal denticles mostly minute, close-set, covering entire body and fins; surfaces mostly smooth to the touch, except for 4 (4-5) slightly enlarged granular denticles on each anterior orbital rim, 1 (1-2) on each inner posterior rim, and 2 (1-2) on the inner edge of each spiracle (Figs. 20 and 24a-d); 53 (4160) enlarged, coarse denticles rather regularly distributed along midline from nape to first dorsal-fin origin (to origin also in most paratypes but to $\sim 60 \mathrm{~mm}$ before origin in adult male paratype $\mathrm{ZMH} 25554$ and to $\sim 10 \mathrm{~mm}$ before origin in juvenile male paratype ZMH 25560; about 10 posteriormost denticles only weakly pronounced in both female ZMH paratypes) (Figs. 24e, i and 25); enlarged denticles absent along midline between dorsal fins and upper caudal origin, on snout tip, anterior and lateral margins of disc, pelvic fins, and bases of dorsal fins; nasal flaps and lamellae naked. Crowns of denticles mostly oval, except largest denticles with slightly erect, bluntly acute cusp, base stellate (Fig. 24a-e). Crowns of denticles in juvenile paratype ZMH 25560 with acutely pointed cusp and a minute cusplet on each side of the cusp (Fig. 24f-i). Furthermore, the denticles of this juvenile paratype are proportionally larger compared to larger specimens and those in midline are set much denser (Fig. $24 \mathrm{i}$ vs. Fig. $24 \mathrm{e}$ and Fig. $25 \mathrm{~b}$ vs. Fig. $25 \mathrm{a}$ ). With respect to the minute dermal denticles covering entire body and fins, those anterior to orbit are tricuspidate in this juvenile paratype with three well-developed, acutely pointed cusps (Fig. 24f). Thorns absent in holotype and paratypes. Prebranchial sensory pore patch distinct, extending to about the level of third gill slit. Post-scapular sensory canal distinct, weakly undulated anteriorly, terminating near pectoral-fin insertions; sensory pores minute, canal not forming a shallow groove (Fig. 15).

Rostral cartilage broad, its shaft just slightly increasing in width posteriorly from rostral node; rostral node rounded at apex, not angular, broadly expanded, relatively short, axis at maximum node width 31.2 (30.7-34.0)\% of length of rostral cartilage from tip; anterior fontanelle relatively broad posteriorly, tapering gradually towards rostral node, dorsolateral edges of cartilage surrounding fontanelle (rostral ridges on surface of snout) well separated posteriorly, not constricting medially; rostral cartilage 60.5 (57.4-61.3)\% of neurocranium length, ventral edges of rostral cartilage united; nasal capsules large, with their transverse axes directed anterolaterally; width of cranium across nasal capsules 1.25 (1.15-1.35) times nasobasal length (base of rostrum to occipital condyles); width of nasal capsule 1.48 (1.39-1.68) times its length; basal plate relatively broad, its minimum width 4.11 (3.41-4.2) times in nasobasal length; cranial roof with small, oval-shaped fenestra, with its anterior edge located behind precerebral cavity by a distance $\sim 1.5$ times its length; anterior cartilage triangular, narrow, posteriorly wedge-shaped, without an anterior lobe extending past nasal capsules; preorbital processes well developed; postorbital processes moderately large, not bifurcate; greatest width across processes 2.02 (1.66-2.11) times in nasobasal length (Fig. 26).

Nasal lamellae 47 left, 48 right (43-48) (left/right orientation based on dorsal view). Pectoral skeleton with 31 left, 30 right (29-32) propterygial, 8 (8-10) mesopterygial, 1 left, 2 right (12) neopterygial, 25 (23-25) metapterygial, amounting to 65 (63-67) total radials. Total pelvic-fin radials 28 (27-31). Total vertebral segment (synarcual and free) counts 194 (195-200); post-synarcual centra 181 (181-186); precaudal centra (excluding synarcual centra) 146 (145-148); synarcual segments 13 (12-14); monospondylous precaudal centra 39 (38-40); diplosondylous precaudal centra 107 (105-109); diplosondylous caudal centra 35 (36-39). Monospondylous to diplosondylous centra transition posterior to pelvic girdle.

\section{Coloration}

Prior to preservation: dorsal surface light to medium brown with a greenish tinge; the patterning with small bluish-gray circular spots is sparse, and such spots are confined to symmetrical patterns on the snout tip, posterior pectoral-fin margins, a pair of tiny spots on midbody behind occipital joint, and few spots on posterior pelvic-fin margins. The spots have rather inconspicuous and very thin brownish margins. Indistinct small (on anterior disc) to larger (on posterior disc and tail) dark brown spots can be found in varying degrees of conspicuousness, sometimes very indistinct, but always 
Table 2 Acroteriobatus stehmanni sp. nov., morphometrics and meristics

\begin{tabular}{|c|c|c|c|c|c|c|c|c|}
\hline & \multicolumn{2}{|c|}{$\begin{array}{l}\text { Acroteriobatus } \\
\text { stehmanni } \text { sp. } \\
\text { nov., adult male } \\
\text { holotype, ZMH } \\
25553\end{array}$} & \multicolumn{2}{|c|}{$\begin{array}{l}\text { Acroteriobatus } \\
\text { stehmanni } \text { sp. } \\
\text { nov., adult female } \\
\text { paratype, ZMH } \\
25557\end{array}$} & \multirow{2}{*}{$\begin{array}{l}\begin{array}{l}\text { Minimum } \\
(n=6)\end{array} \\
\% \mathrm{TL}\end{array}$} & \multirow{2}{*}{$\begin{array}{l}\text { Maximum } \\
(n=6)\end{array}$} & \multirow{2}{*}{$\begin{array}{l}\text { Mean } \\
(n=8)\end{array}$} & \multirow[t]{2}{*}{ SD } \\
\hline & $\mathrm{mm}$ & $\% \mathrm{TL}$ & $\mathrm{mm}$ & $\% \mathrm{TL}$ & & & & \\
\hline Total length (mm) & 597.0 & 100.0 & 603.0 & 100.0 & 201.4 & 613.0 & 511.3 & \\
\hline Disc width, max & 175.0 & 29.3 & 190.2 & 31.5 & 28.5 & 35.9 & 30.9 & 2.5 \\
\hline Disc length, direct/point-to-point (ptp) & 228.0 & 38.2 & 241.3 & 40.0 & 37.6 & 45.2 & 39.7 & 2.5 \\
\hline Head length, dorsal (to occipital joint) & 106.0 & 17.8 & 108.1 & 17.9 & 17.2 & 22.8 & 18.5 & 1.8 \\
\hline Head length, ventral (to 5 th gill slit), ptp & 145.5 & 24.4 & 144.0 & 23.9 & 23.6 & 29.7 & 25.1 & 2.0 \\
\hline Head length, ventral (to 5th gill slit), horizontal & 143.9 & 24.1 & 142.3 & 23.6 & 23.2 & 29.4 & 24.8 & 2.0 \\
\hline Snout length, direct (pre-eyeball) & 80.4 & 13.5 & 78.9 & 13.1 & 13.1 & 17.3 & 14.0 & 1.4 \\
\hline Snout length, horizontal (pre-eyeball) & 78.6 & 13.2 & 76.3 & 12.6 & 12.7 & 16.5 & 13.6 & 1.2 \\
\hline Snout length, presocket & 77.6 & 13.0 & 75.5 & 12.5 & 12.2 & 16.3 & 13.4 & 1.2 \\
\hline Orbit diameter, $\mathrm{ptp}$ & 20.6 & 3.5 & 22.0 & 3.6 & 3.3 & 4.6 & 3.7 & 0.4 \\
\hline Spiracle length & 11.5 & 1.9 & 11.9 & 2.0 & 1.6 & 2.5 & 2.0 & 0.2 \\
\hline Orbit and spiracle length & 26.9 & 4.5 & 28.1 & 4.7 & 4.1 & 5.7 & 4.6 & 0.5 \\
\hline Interorbital width 1 (skeletal) & 17.9 & 3.0 & 17.9 & 3.0 & 3.1 & 4.1 & 3.3 & 0.4 \\
\hline Interorbital width 2 (= intereye width) & 37.2 & 6.2 & 37.1 & 6.2 & 5.9 & 8.6 & 6.6 & 0.9 \\
\hline Interspiracular width & 27.9 & 4.7 & 28.1 & 4.7 & 4.5 & 6.3 & 5.0 & 0.6 \\
\hline Preoral length & 94.7 & 15.9 & 90.2 & 15.0 & 14.9 & 19.2 & 16.0 & 1.4 \\
\hline MOW, mouth width & 32.2 & 5.4 & 32.1 & 5.3 & 5.2 & 6.7 & 5.6 & 0.5 \\
\hline Prenarial length, ptp & 73.1 & 12.2 & 71.7 & 11.9 & 11.7 & 15.6 & 12.7 & 1.3 \\
\hline NOW, nostril length & 20.5 & 3.4 & 18.1 & 3.0 & 3.0 & 3.4 & 3.2 & 0.2 \\
\hline AAW, anterior nasal aperture, width & 6.1 & 1.0 & 6.6 & 1.1 & 1.0 & 1.2 & 1.1 & 0.1 \\
\hline ANF, anterior nasal flap, base length & 17.8 & 3.0 & 16.9 & 2.8 & 2.8 & 3.8 & 3.0 & 0.3 \\
\hline ANW, anterior nasal flap, width & 9.5 & 1.6 & 9.2 & 1.5 & 1.5 & 1.7 & 1.6 & 0.1 \\
\hline PLT, posterolateral nasal flap, total length & 16.8 & 2.8 & 15.2 & 2.5 & 2.6 & 2.8 & 2.7 & 0.1 \\
\hline PLF, posterolateral nasal-flap anterior exposed base length & 7.1 & 1.2 & 7.3 & 1.2 & 1.1 & 1.9 & 1.3 & 0.2 \\
\hline PLW, posterolateral nasal flap, width & 4.3 & 0.7 & 4.3 & 0.7 & 0.6 & 1.1 & 0.8 & 0.2 \\
\hline PNF, posterior nasal flap, base length & 11.7 & 2.0 & 10.7 & 1.8 & 1.9 & 2.1 & 1.9 & 0.1 \\
\hline PNW, posterior nasal flap, width & 5.4 & 0.9 & 5.1 & 0.9 & 0.8 & 1.1 & 0.9 & 0.1 \\
\hline INM, distance between lateral margins of anterior apertures & 49.4 & 8.3 & 49.3 & 8.2 & 8.3 & 9.8 & 8.6 & 0.5 \\
\hline INW, distance between posterior margins of nostrils & 13.7 & 2.3 & 14.8 & 2.5 & 2.3 & 3.5 & 2.6 & 0.4 \\
\hline INA, distance between insertions of anterior nasal flaps & 6.0 & 1.0 & 5.6 & 0.9 & 0.8 & 1.1 & 1.0 & 0.1 \\
\hline Shortest direct distance from anterior opening of nostril to disc margin & 20.3 & 3.4 & 23.9 & 4.0 & 3.5 & 5.7 & 4.0 & 0.8 \\
\hline Gill opening width, 1st & 7.7 & 1.3 & 7.5 & 1.2 & 1.0 & 1.4 & 1.3 & 0.1 \\
\hline Gill opening width, 2nd & 7.7 & 1.3 & 8.1 & 1.3 & 1.1 & 1.5 & 1.3 & 0.1 \\
\hline Gill opening width, 3rd & 7.7 & 1.3 & 8.4 & 1.4 & 1.2 & 1.7 & 1.3 & 0.2 \\
\hline Gill opening width, 4th & 7.4 & 1.2 & 7.9 & 1.3 & 1.2 & 1.5 & 1.3 & 0.1 \\
\hline Gill opening width, 5 th & 5.2 & 0.9 & 6.1 & 1.0 & 0.9 & 1.1 & 1.0 & 0.1 \\
\hline Distance between first gill openings & 64.9 & 10.9 & 71.1 & 11.8 & 10.9 & 14.5 & 11.8 & 1.2 \\
\hline Distance between fifth gill openings & 45.9 & 7.7 & 51.2 & 8.5 & 7.5 & 9.8 & 8.2 & 0.8 \\
\hline Pelvic fin, length & 99.1 & 16.6 & 108.8 & 18.0 & 15.8 & 17.6 & 16.8 & 0.7 \\
\hline Pelvic fin, anterior margin length & 55.0 & 9.2 & 58.8 & 9.8 & 8.5 & 10.0 & 9.4 & 0.5 \\
\hline Pelvic fin, width & 33.8 & 5.7 & 43.2 & 7.2 & 4.9 & 6.9 & 6.0 & 0.8 \\
\hline Pelvic fin, base length & 53.1 & 8.9 & 69.8 & 11.6 & 7.2 & 11.1 & 9.5 & 1.3 \\
\hline Pelvic fin, inner margin length & 53.1 & 8.9 & 47.6 & 7.9 & 7.9 & 8.9 & 8.5 & 0.5 \\
\hline First dorsal fin, length & 39.2 & 6.6 & 38.3 & 6.3 & 6.3 & 6.9 & 6.6 & 0.2 \\
\hline
\end{tabular}


Table 2 (continued)

\begin{tabular}{|c|c|c|c|c|c|c|c|c|}
\hline & \multicolumn{2}{|c|}{$\begin{array}{l}\text { Acroteriobatus } \\
\text { stehmanni } \text { sp. } \\
\text { nov., adult male } \\
\text { holotype, ZMH } \\
25553\end{array}$} & \multicolumn{2}{|c|}{$\begin{array}{l}\text { Acroteriobatus } \\
\text { stehmanni } \text { sp. } \\
\text { nov., adult female } \\
\text { paratype, ZMH } \\
25557\end{array}$} & \multirow{2}{*}{$\begin{array}{l}\begin{array}{l}\text { Minimum } \\
(n=6)\end{array} \\
\% \mathrm{TL}\end{array}$} & \multirow{2}{*}{$\begin{array}{l}\begin{array}{l}\text { Maximum } \\
(n=6)\end{array} \\
\% \mathrm{TL}\end{array}$} & \multirow{2}{*}{$\begin{array}{l}\text { Mean } \\
(n=8)\end{array}$} & \multirow[t]{2}{*}{ SD } \\
\hline & $\mathrm{mm}$ & $\% \mathrm{TL}$ & $\mathrm{mm}$ & $\% \mathrm{TL}$ & & & & \\
\hline First dorsal fin, anterior margin length & 53.6 & 9.0 & 55.0 & 9.1 & 8.8 & 10.0 & 9.2 & 0.4 \\
\hline First dorsal fin, height & 39.2 & 6.6 & 42.3 & 7.0 & 5.8 & 7.6 & 6.7 & 0.5 \\
\hline First dorsal fin, base length & 29.2 & 4.9 & 25.2 & 4.2 & 4.2 & 5.0 & 4.6 & 0.3 \\
\hline First dorsal fin, inner margin length & 10.6 & 1.8 & 11.6 & 1.9 & 1.7 & 2.6 & 2.0 & 0.3 \\
\hline Second dorsal fin, length & 40.4 & 6.8 & 39.0 & 6.5 & 6.4 & 7.1 & 6.7 & 0.2 \\
\hline Second dorsal fin, anterior margin length & 49.5 & 8.3 & 52.8 & 8.8 & 8.3 & 9.9 & 8.8 & 0.5 \\
\hline Second dorsal fin, height & 36.1 & 6.1 & 38.9 & 6.4 & 5.9 & 7.0 & 6.3 & 0.4 \\
\hline Second dorsal fin, base length & 31.1 & 5.2 & 28.7 & 4.8 & 4.6 & 5.3 & 5.0 & 0.2 \\
\hline Second dorsal fin, inner margin length & 10.2 & 1.7 & 10.8 & 1.8 & 1.5 & 2.3 & 1.8 & 0.3 \\
\hline Caudal fin, dorsal margin & 71.5 & 12.0 & 69.2 & 11.5 & 10.9 & 14.6 & 12.0 & 1.1 \\
\hline Caudal fin, preventral margin & 47.7 & 8.0 & 42.1 & 7.0 & 7.0 & 8.4 & 7.7 & 0.5 \\
\hline Snout to pelvic-fin origin ptp & 207.2 & 34.7 & 215.7 & 35.8 & 32.9 & 40.7 & 35.5 & 2.4 \\
\hline Snout to anterior vent & 228.7 & 38.3 & 241.2 & 40.0 & 37.1 & 43.8 & 39.4 & 2.2 \\
\hline Snout to first dorsal-fin origin & 353.0 & 59.1 & 370.0 & 61.4 & 58.4 & 61.8 & 60.0 & 1.3 \\
\hline Snout to second dorsal-fin origin & 455.0 & 76.2 & 462.0 & 76.6 & 75.0 & 77.5 & 76.4 & 0.7 \\
\hline Snout to upper caudal-fin origin & 525.0 & 87.9 & 530.0 & 87.9 & 86.4 & 88.8 & 87.7 & 0.8 \\
\hline Snout to lower caudal-fin origin & 530.0 & 88.8 & 533.0 & 88.4 & 86.2 & 89.1 & 88.3 & 1.0 \\
\hline Pelvic-fin insertion to dorsal-fin origin & 96.1 & 16.1 & 89.2 & 14.8 & 13.0 & 16.2 & 15.1 & 1.2 \\
\hline Interdorsal distance & 74.2 & 12.4 & 68.6 & 11.4 & 10.8 & 13.1 & 12.0 & 0.8 \\
\hline Disc width, anterior orbit & 103.5 & 17.3 & 103.5 & 17.2 & 16.7 & 22.9 & 18.2 & 2.0 \\
\hline Body width, pelvic insertion & 58.1 & 9.7 & 59.7 & 9.9 & 8.2 & 10.9 & 9.9 & 0.7 \\
\hline Body width, first dorsal-fin origin & 55.4 & 9.3 & 59.0 & 9.8 & 7.5 & 10.4 & 9.5 & 0.9 \\
\hline Body width, second dorsal-fin origin & 31.1 & 5.2 & 32.9 & 5.4 & 4.5 & 5.7 & 5.3 & 0.4 \\
\hline Body depth, anterior orbit & 18.4 & 3.1 & 20.5 & 3.4 & 3.0 & 3.8 & 3.3 & 0.3 \\
\hline Body depth, maximum & 25.7 & 4.3 & 27.5 & 4.6 & 4.2 & 5.3 & 4.7 & 0.4 \\
\hline Body depth, pelvic-fin insertion & 25.4 & 4.3 & 26.7 & 4.4 & 3.7 & 4.8 & 4.4 & 0.4 \\
\hline Body depth, first dorsal-fin origin & 21.0 & 3.5 & 21.3 & 3.5 & 2.9 & 3.8 & 3.5 & 0.3 \\
\hline Body depth, second dorsal-fin origin & 14.2 & 2.4 & 14.7 & 2.4 & 2.1 & 2.5 & 2.4 & 0.1 \\
\hline Body depth, caudal peduncle & 9.0 & 1.5 & 10.2 & 1.7 & 1.5 & 1.6 & 1.6 & 0.1 \\
\hline Caudal peduncle length $=$ dorsal $(\mathrm{D} 2)$ - caudal space & 40.0 & 6.7 & 39.6 & 6.6 & 6.1 & 6.9 & 6.5 & 0.3 \\
\hline Tail length from anterior vent & 368.3 & 61.7 & 361.8 & 60.0 & 56.2 & 62.9 & 60.6 & 2.2 \\
\hline Angle before eyes, ${ }^{\circ}$ & \multicolumn{2}{|c|}{72.0} & \multicolumn{2}{|c|}{77.0} & 71.0 & 75.0 & 73.0 & 2.0 \\
\hline
\end{tabular}

Individual values for the adult male holotype (ZMH 25553) and one adult female paratype (ZMH 25557), ranges for all other ZMH paratypes $(n=6)$, as well as means and standard deviations (SD) for all eight ZMH type specimens are presented. Proportional values are expressed as percentages of total length (TL) $70 \%$ ethanol preserved except for minimum, maximum, and mean of TL in $\mathrm{mm}$

arranged in symmetrical patterns, extending also onto dorsal and caudal fins (Figs. 16 and 17). Snout semi-translucent with a few, somewhat elongated bluish-gray spots only giving it a very reduced stripe-nosed appearance (Figs. 16 and 17). Outer edges of pelvic-fin (and partially posterior pectoral-fin) margins, as well as lateral tail folds conspicuously white (Figs. 16 and 17). Ventral surface uniformly white. After preservation: bluish-gray spots mostly not visible anymore, at most few detectable on posterior pectoral-fin margins; brownish spots more or less faded, some specimens, including one of the females with fresh coloration described above, show almost no dark spots anymore. Outer edges of pelvic-fin (and partially posterior pectoral-fin) margins, as well as lateral tail folds creamy instead of white as typical for preserved 
specimens (Fig. 14). Ventral surface uniformly creamy as well (Fig. 14); smallest juvenile paratype ZMH 25560 with prominent blackish blotch on underside of snout tip plus two tiny black spots on outer edges of anterior snout about half way from snout tip to nostrils (Fig. 27).

\section{Size}

The new species is described from 10 specimens (seven males and three females), ranging in size from 208 to $622 \mathrm{~mm} \mathrm{TL}$ (fresh) and from 201 to $613 \mathrm{~mm}$ TL (preserved). Adult males range from 546 to $622 \mathrm{~mm}$ TL (fresh), a $378 \mathrm{~mm}$ TL male is a very early subadult specimen, and a $208 \mathrm{~mm}$ TL male is juvenile. Accordingly, males of the new species apparently start to mature at around $378 \mathrm{~mm}$ TL. The two female paratypes of $594 \mathrm{~mm}$ and $602 \mathrm{~mm}$ TL are presumed to be adult, considering that they are distinctly larger than the smallest adult male paratypes. Based on all known specimens, the new species is assumed to be a small Acroteriobatus species reaching only about $622 \mathrm{~mm}$ TL.

\section{Distribution}

Acroteriobatus stehmanni sp. nov. is presently only known from the coastal waters surrounding the Socotra Islands and may be endemic to this region (Fig. 13). It is known from 36 to $43 \mathrm{~m}$ depth.

\section{Etymology}

The species is named in honor of Dr. Matthias F.W. Stehmann for his invaluable contributions to chondrichthyan taxonomy, particularly with respect to skates. He taught SW and BS chondrichthyan taxonomy, collected all type specimens of the new species, and kindly provided fresh photographs of two of them.
Fig. 28 Acroteriobatus

leucospilus, BMNH 1905.6.8.12, subadult male syntype, $416 \mathrm{~mm}$ $\mathrm{TL}$, in total dorsal view. The photograph was taken and kindly provided by Kevin Webb
Fig. 29 Acroteriobatus leucospilus, BMNH 1905.6.8.12, subadult male syntype, $416 \mathrm{~mm}$ $\mathrm{TL}$, in total ventral view. The photograph was taken and kindly provided by Kevin Webb
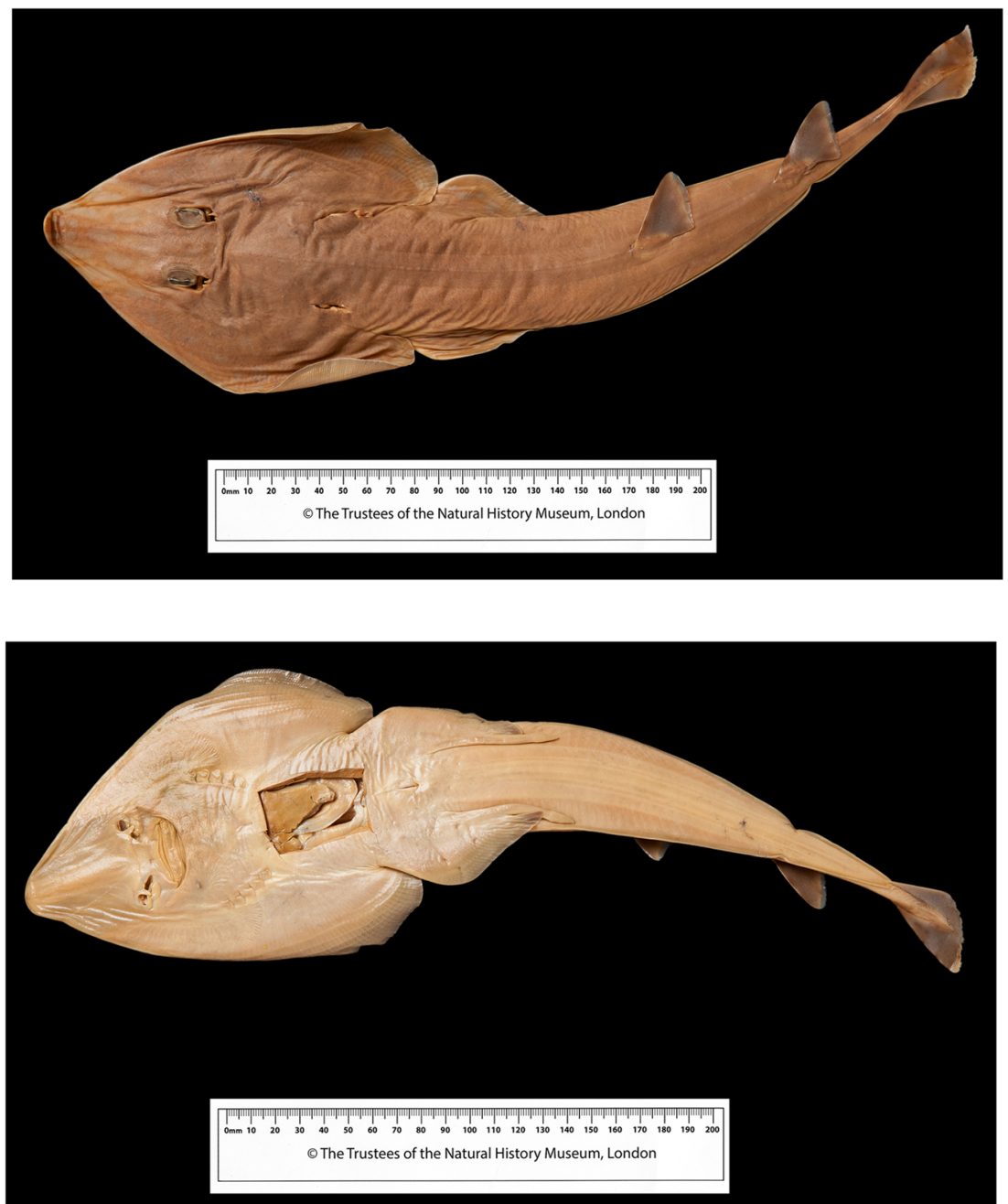
Fig. 30 Acroteriobatus leucospilus, SAIAB 34588, gravid female, $690 \mathrm{~mm}$ TL, in total dorsal view. Photograph courtesy Marsha Englebrecht (C)

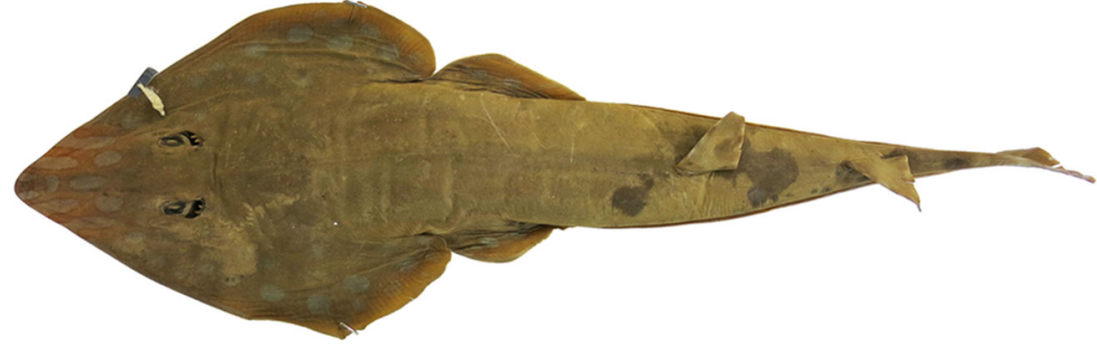

Fig. 31 Acroteriobatus leucospilus, underwater photograph showing the vivid live coloration. The photograph was taken and kindly provided by Dennis King

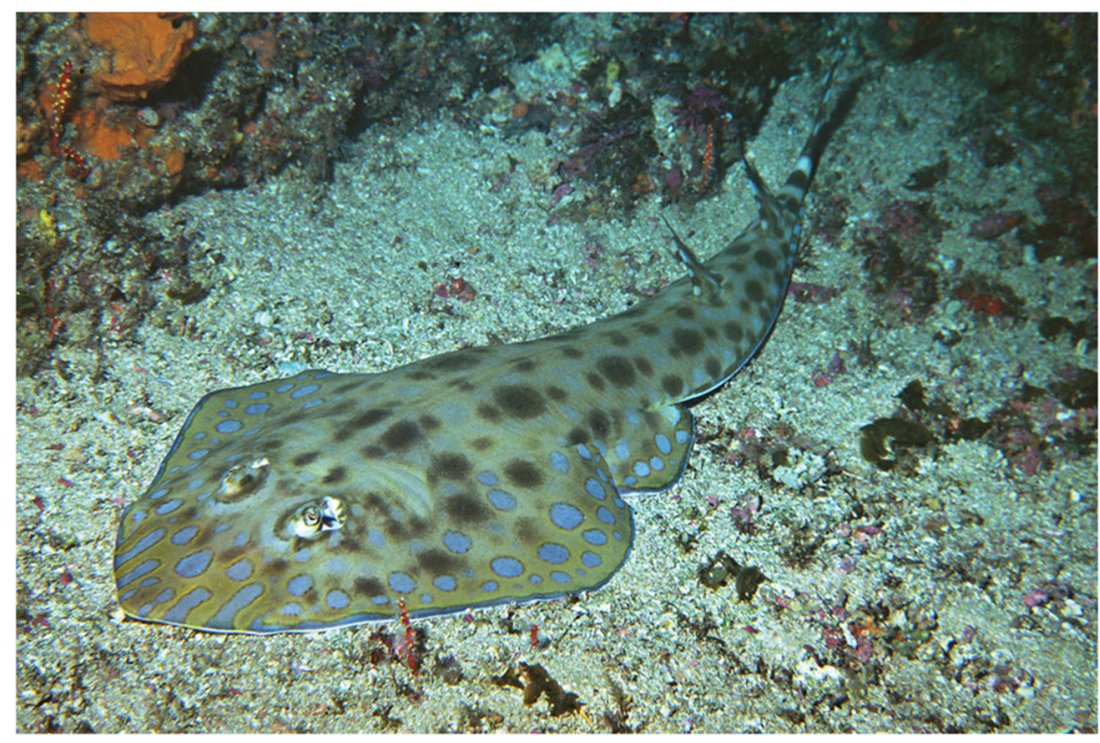

Rhinobatus blochii (not Müller \& Henle)-Regan (1908:

Acroteriobatus leucospilus (Norman, 1926)

(Blue-spotted guitarfish)

(Figs 28, 29, 30, 31, 32, 33, 34, 35, 36, and 37, Table 3)

Acroteriobatus leucospilus-Ebert and van Hees (2015: 146); Weigmann (2016: 920); Séret et al. (2016: 81); Séret and de Carvalho (in press); Séret (in press)

Rhinobatus annulatus (not Müller \& Henle)-Smith (1961: 64 [in part], not pl. 3)

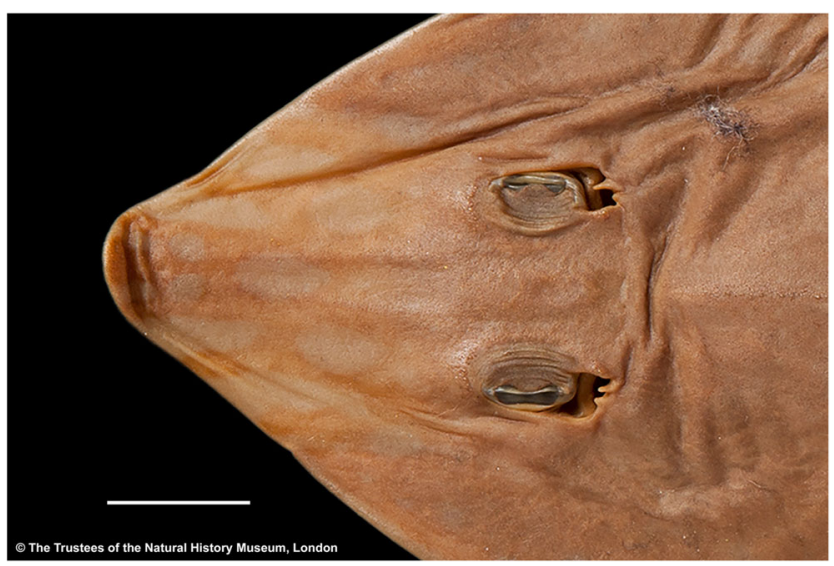

Fig. 32 Acroteriobatus leucospilus, BMNH 1905.6.8.12, subadult male syntype, $416 \mathrm{~mm}$ TL, head in dorsal view. Scale bar: $2 \mathrm{~cm}$. The photograph was taken and kindly provided by Kevin Webb
242) (in part); Gilchrist \& Thompson (1911: 55); Gilchrist \& Thompson (1916: 284) (in part); Smith (1961: not 64, pl. 3)

Rhinobatus blochi-Barnard (1925: 61) (in part)

Rhinobatos leucospilus-Fowler (1941: 313) and subsequent authors

Rhinobatus leucospilus-Norman (1926: 966); Barnard (1927: 1014-1015)

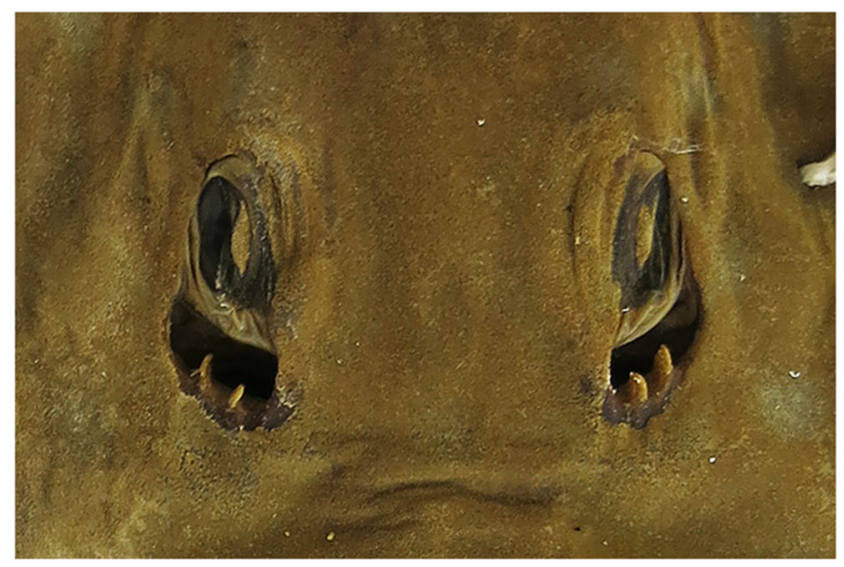

Fig. 33 Acroteriobatus leucospilus, SAIAB 34588, gravid female, $690 \mathrm{~mm}$ TL, close-up of orbital and spiracular region. Photograph courtesy Marsha Englebrecht (C) 


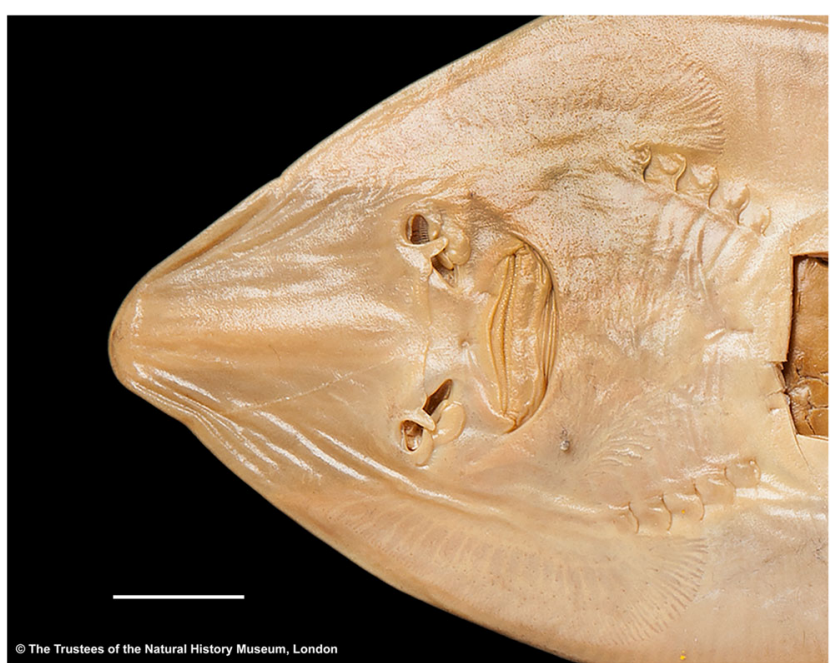

Fig. 34 Acroteriobatus leucospilus, BMNH 1905.6.8.12, subadult male syntype, $416 \mathrm{~mm}$ TL, head in ventral view. Scale bar: $2 \mathrm{~cm}$. The photograph was taken and kindly provided by Kevin Webb

Syntypes BMNH 1905.6.8.12, subadult male, $416 \mathrm{~mm}$ TL, and BMNH 1920.7.23.1, juvenile male, $248 \mathrm{~mm}$ TL, Durban, KwaZulu-Natal, South Africa, southwestern Indian Ocean.

Non-types South Africa: SAIAB 189087, 223 mm TL (after preservation), juvenile male, Tugela Bank, KwaZuluNatal, South Africa, $29^{\circ} 7.5^{\prime} \mathrm{S}, 31^{\circ} 45^{\prime} \mathrm{E}, 15$ March 2009; SAIAB 34588, $690 \mathrm{~mm}$ TL (after preservation), gravid female, KwaZulu-Natal, South Africa, 23 October 1982; SAIAB 11142 (formerly ORI B 90), $960 \mathrm{~mm}$ TL (after preservation), adult female, Tugela Bank, KwaZulu-Natal, South Africa; SAIAB 11143 (formerly ORI B 112), $650 \mathrm{~mm}$ TL (after preservation), adult female, Tugela Bank, KwaZulu-Natal, South

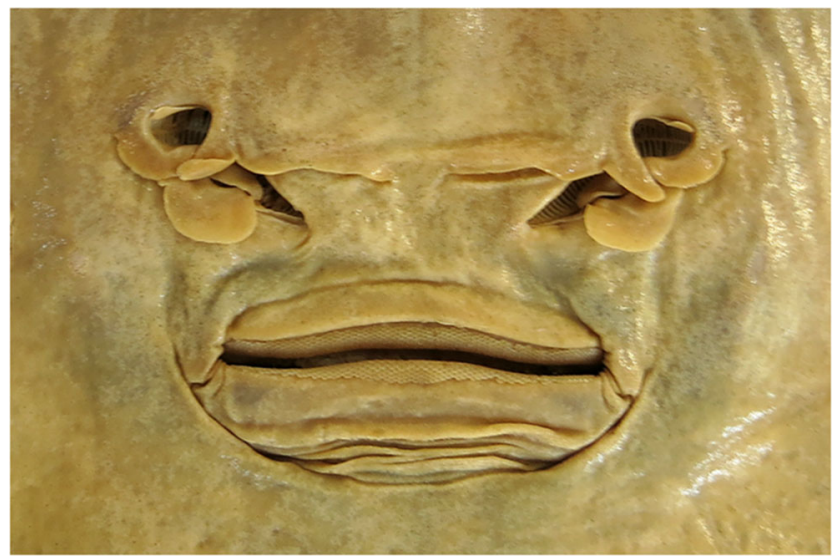

Fig. 35 Acroteriobatus leucospilus, SAIAB 34588, gravid female, $690 \mathrm{~mm}$ TL, close-up of mouth-nasal region. Photograph courtesy Marsha Englebrecht (C)
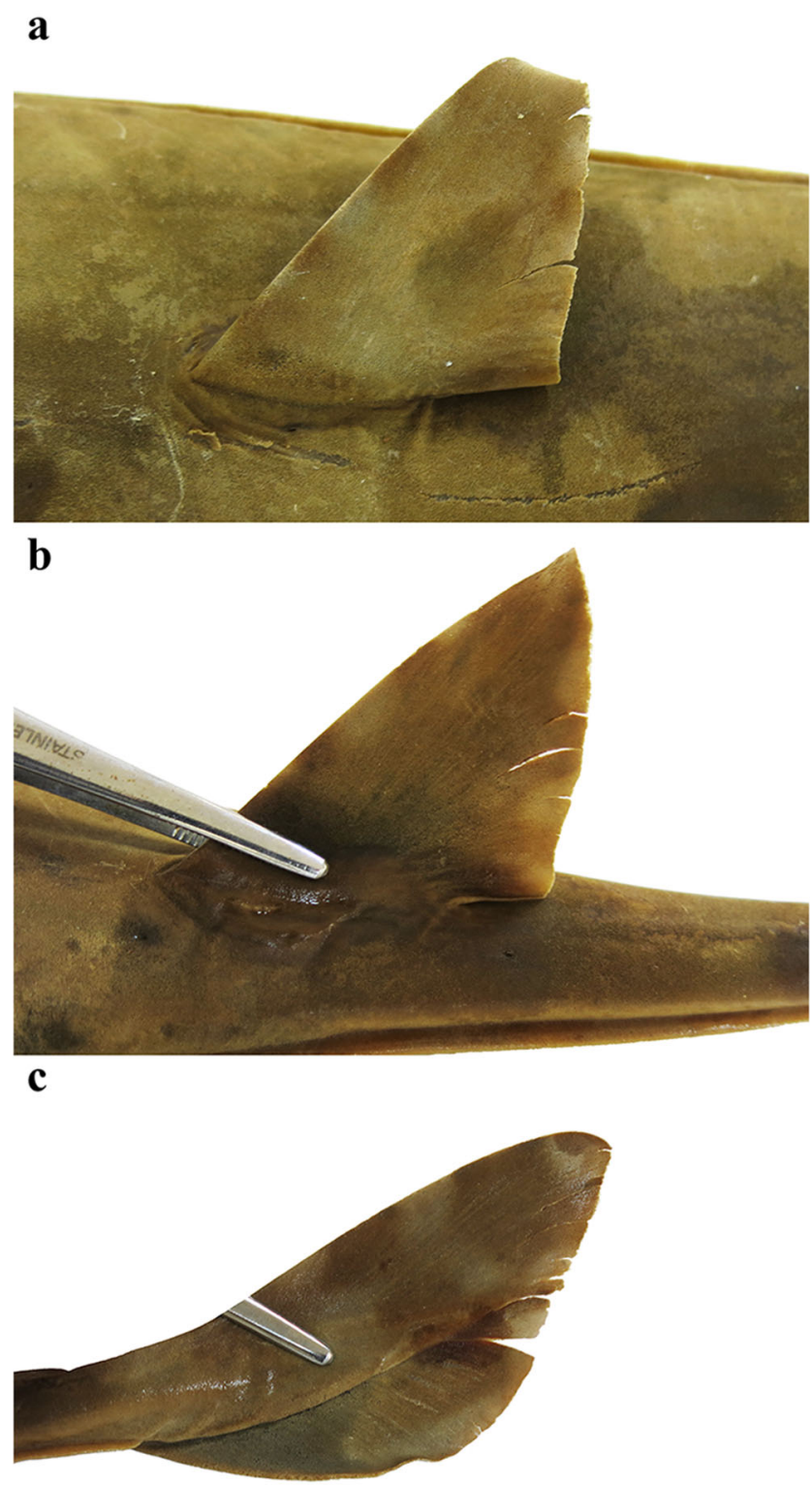

Fig. 36 Acroteriobatus leucospilus, SAIAB 34588, gravid female, $690 \mathrm{~mm}$ TL; first dorsal (a), second dorsal (b), and caudal (c) fins in lateral views. Photograph courtesy Marsha Englebrecht $(\odot)$

Africa; SAIAB 11159 (formerly ORI B 799), $295 \mathrm{~mm}$ TL (after preservation), juvenile female, Tugela Bank, KwaZulu-Natal, South Africa.

Mozambique: SAIAB 40000, $430 \mathrm{~mm}$ TL (after preservation), subadult male, Maputo Bay, Mozambique, $25.96666717^{\circ} \mathrm{S}, 32.5833320618^{\circ} \mathrm{E}, 29$ May 1992; SAIAB 55691, $445 \mathrm{~mm}$ TL (after preservation), juvenile female, Moebase fishing village, Mozambique, $17.04999923^{\circ} \mathrm{S}$, $39.7333335876^{\circ}$ E, 12 July 1997; SAIAB $12829,515 \mathrm{~mm}$ TL (after preservation), juvenile female, Inhaca Island, 


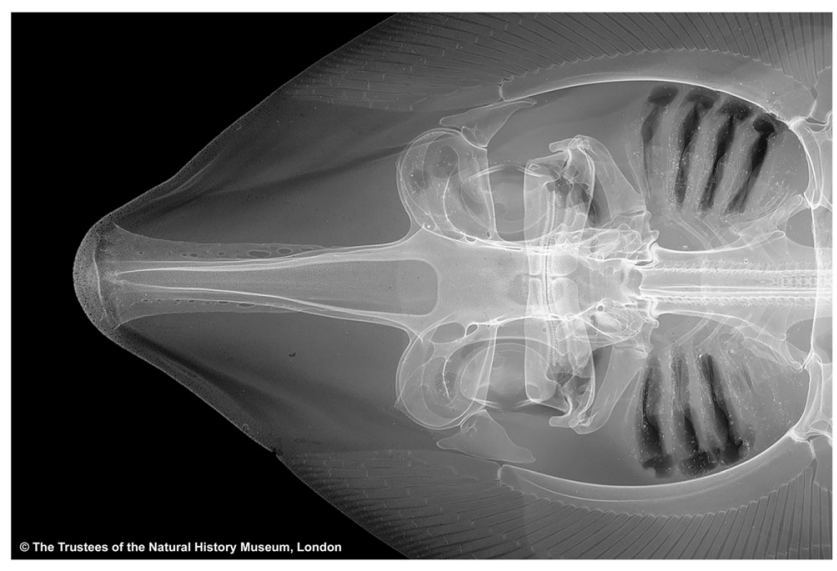

Fig. 37 Acroteriobatus leucospilus, BMNH 1905.6.8.12, subadult male syntype, $416 \mathrm{~mm}$ TL, radiograph of cranium and snout in dorsal view. The radiograph was taken and kindly provided by James Maclaine and reworked by Nemo Martin

Mozambique, $26.01666641^{\circ} \mathrm{S}, 32.96666717^{\circ} \mathrm{E}$, October 1953.

Tanzania: SAIAB 12162, $629 \mathrm{~mm}$ TL (after preservation), female, Zanzibar Island, Tanzania, $6.166666984^{\circ} \mathrm{S}$, $39.1833343506^{\circ} \mathrm{E}$.

\section{Diagnosis}

A medium-sized Acroteriobatus species distinguished by the following combination of characters: dorsal surface smooth, without prominent thorns or tubercles, except for slightly enlarged granular denticles partially around orbital rims and rather regularly distributed along midline from nape to near first dorsal-fin origin; absent between dorsal fins and upper caudal fin. Snout semi-translucent with elongated bluish spots giving stripe-nosed appearance, numerous small bluish spots covering snout, pectoral, pelvic, dorsal, and caudal fins but not central disc on a sandy brown background with darker brown spots of varying sizes covering the disc, tail, fin bases, and fins, sometimes giving the caudal peduncle a striped appearance; outer edges of pectoral- and pelvic-fin margins blue, lateral tail folds white or striped blue and brown; ventral surface white. Nasal lamellae 37-41; upper jaw tooth row count $\sim 60-75 ; 187-192$ post-synarcual centra; 200-205 total vertebral segments; $63-70$ total pectoral skeleton radials.

\section{Description}

The description is based on the two syntypes (BMNH 1905.6.8.12 and BMNH 1920.7.23.1), as well as nine nontype specimens. Where relevant, ratios are based on horizontal measurements unless otherwise stated. Detailed morphometric measurements and meristics are given in Table 3.
External morphology (Figs. 28, 29, 30, 31, 32, 33, 34, 35, and 36) Disc wedge-shaped, narrowly angular anteriorly, snout angle before eyes moderately acute, $68.0-80.5^{\circ}$; anterior margin straight, outer pectoral margin broadly rounded, posterior margin convex, rear tip broadly rounded; disc length 1.15 1.24 times its width. Pelvic fins elongate, short-based, base length 0.89-2.04 times inner margin length; pelvic-fin total length 1.44-2.12 times base length, 2.19-3.06 times width; anterior margin straight, becoming weakly convex towards broadly rounded apex, posterior margin weakly convex, free rear tip narrowly rounded. Tail elongate, slightly constricted in anterior part between pelvic-fin origin and about level pelvic-fin free rear tip, broadest somewhat anterior to first dorsal-fin origin, tapering posteriorly; very strongly depressed dorsoventrally, in cross section nearly flat below, more rounded above; tail length from anterior cloaca 1.35-1.62 times precloacal length, 1.31-1.66 times disc length, and 5.20 6.03 times body width at pelvic-fin insertions; body width 2.17-2.67 times depth at pelvic-fin insertions, 2.39-3.26 at first dorsal-fin origin, 1.74-2.44 at second dorsal-fin origin. Dermal fold lateral along tail, originating about level pelvicfin free rear tip and reaching onto base of caudal fin just behind ventral origin of caudal fin; fold well developed, maximum width at interdorsal space more than half width of posterior nasal flap and more than length of longest spiracular fold (Figs. 28, 29, 30, and 31).

Head moderately elongate, ventral length $22.9-28.3 \% \mathrm{TL}$, dorsal length $24.0-29.2 \%$ TL; snout rather short, bluntly pointed, preoral length 2.22-2.76 times mouth width, 4.285.08 times internarial distance, $1.09-1.54$ times dorsal caudalfin margin, 2.59-3.46 times distance from nostril to margin of disc; direct preorbital snout length 2.17-2.6 times interspiracular distance, 3.3-5.36 times orbit diameter, 2.653.67 times skeletal interorbital space; interorbital space weakly concave, rather narrow; eyes moderately large, slightly elevated, but not protruding, orbit diameter 1.07-2.0 times spiracle length, 0.58-1.11 times skeletal interorbital space. Spiracles relatively large, narrowly bean-shaped; two prominent and strongly compressed folds on upper posterior margin, length of inner spiracular fold $0.5-0.75$ times that of outer fold; distance between bases of folds 0.25-1.0 times length of shortest fold (Figs. 32 and 33).

Nostril moderately large, oblique, all nasal flaps well developed; anterior aperture subcircular; nostril length 1.7-3.0 times anterior aperture width, $0.75-1.13$ times anterior nasalflap base length, 0.5-0.94 times distance from nostril to edge of disc, 0.81-1.31 times internarial distance. Anterior nasalflap base greatly extended, penetrating horizontally into internarial space, mesial edges barely separated (by about width of posterior nasal flap); innermost extension of flap not narrow; flap base length 1.88-2.86 times its width at process, 1.88-3.33 times anterior aperture width; minimum distance between mesial insertions of flaps 6.75-26.5 in greatest 
Table 3 Acroteriobatus leucospilus, morphometrics and meristics

\begin{tabular}{|c|c|c|c|c|c|c|c|c|}
\hline & \multicolumn{2}{|c|}{$\begin{array}{l}\text { Acroteriobatus } \\
\text { leucospilus, } \\
\text { subadult male } \\
\text { syntype, BMNH } \\
1905.6 .8 .12\end{array}$} & \multicolumn{2}{|c|}{$\begin{array}{l}\text { Acroteriobatus } \\
\text { leucospilus, } \\
\text { juvenile male } \\
\text { syntype, BMNH } \\
\text { 1920.7.23.1 }\end{array}$} & \multirow{2}{*}{$\begin{array}{l}\text { Minimum } \\
(n=9)\end{array}$} & \multirow{2}{*}{ 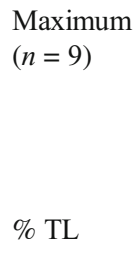 } & \multirow{2}{*}{$\begin{array}{l}\begin{array}{l}\text { Mean } \\
(n=11)\end{array} \\
\% \mathrm{TL}\end{array}$} & \multirow[t]{2}{*}{ SD } \\
\hline & $\mathrm{mm}$ & $\% \mathrm{TL}$ & $\mathrm{mm}$ & $\% \mathrm{TL}$ & & & & \\
\hline Total length (mm) & 416.0 & 100.0 & 248.0 & 100.0 & 223.0 & 960.0 & 498.8 & \\
\hline Disc width, max & 137.0 & 32.9 & 91.0 & 36.7 & 31.5 & 36.3 & 33.3 & 1.8 \\
\hline Disc length, direct/point-to-point (ptp) & 166.0 & 39.9 & 105.0 & 42.3 & 37.0 & 43.9 & 40.0 & 1.8 \\
\hline Head length, dorsal (to occipital joint) & 110.4 & 26.5 & 65.8 & 26.5 & 24.0 & 29.2 & 27.0 & 1.5 \\
\hline Head length, ventral (to 5th gill slit), ptp & 102.4 & 24.6 & 67.0 & 27.0 & 22.9 & 28.3 & 25.1 & 1.7 \\
\hline Snout length, presocket & 55.5 & 13.3 & 31.4 & 12.6 & 10.6 & 14.8 & 12.6 & 1.0 \\
\hline Orbit diameter, ptp & 13.6 & 3.3 & 7.5 & 3.0 & 2.3 & 4.5 & 2.9 & 0.6 \\
\hline Spiracle length & 10.5 & 2.5 & 6.9 & 2.8 & 2.0 & 2.4 & 2.2 & 0.2 \\
\hline Orbit and spiracle length & 18.2 & 4.4 & 12.4 & 5.0 & 4.0 & 6.3 & 4.7 & 0.6 \\
\hline Interorbital width 1 (skeletal) & 15.4 & 3.7 & 10.0 & 4.0 & 3.5 & 4.7 & 3.9 & 0.3 \\
\hline Interspiracular width & 21.7 & 5.2 & 14.4 & 5.8 & 4.7 & 6.3 & 5.2 & 0.5 \\
\hline Preoral length & 62.7 & 15.1 & 40.7 & 16.4 & 12.5 & 17.5 & 14.6 & 1.5 \\
\hline MOW, mouth width & 25.0 & 6.0 & 15.4 & 6.2 & 5.3 & 7.2 & 5.9 & 0.5 \\
\hline Prenarial length, ptp & 50.1 & 12.0 & 31.1 & 12.5 & 9.9 & 13.9 & 11.9 & 1.0 \\
\hline NOW, nostril length & 12.2 & 2.9 & 8.7 & 3.5 & 2.4 & 4.0 & 3.2 & 0.6 \\
\hline AAW, anterior nasal aperture, width & 4.7 & 1.1 & 3.1 & 1.3 & 0.9 & 1.8 & 1.3 & 0.3 \\
\hline ANF, anterior nasal flap, base length & 12.0 & 2.9 & 9.3 & 3.8 & 2.4 & 3.9 & 3.3 & 0.6 \\
\hline ANW, anterior nasal flap, width & 5.8 & 1.4 & 4.1 & 1.7 & 1.2 & 1.9 & 1.5 & 0.2 \\
\hline PLT, posterolateral nasal flap, total length & 9.7 & 2.3 & 4.8 & 1.9 & 1.8 & 2.7 & 2.2 & 0.3 \\
\hline PLW, posterolateral nasal flap, width & 2.3 & 0.6 & 2.4 & 1.0 & 0.4 & 0.9 & 0.6 & 0.2 \\
\hline PNF, posterior nasal flap, base length & 7.2 & 1.7 & 4.8 & 1.9 & 1.2 & 2.7 & 1.8 & 0.4 \\
\hline PNW, posterior nasal flap, width & 3.9 & 0.9 & 2.2 & 0.9 & 0.7 & 1.3 & 0.9 & 0.2 \\
\hline INM, distance between lateral margins of anterior apertures & 36.5 & 8.8 & 24.3 & 9.8 & 6.9 & 10.8 & 8.7 & 1.1 \\
\hline INW, distance between posterior margins of nostrils & 13.3 & 3.2 & 9.5 & 3.8 & 2.6 & 3.6 & 3.1 & 0.4 \\
\hline INA, distance between insertions of anterior nasal flaps & 1.8 & 0.4 & 1.0 & 0.4 & 0.3 & 1.2 & 0.6 & 0.3 \\
\hline Shortest direct distance from anterior opening of nostril to disc margin & 18.1 & 4.4 & 13.1 & 5.3 & 4.2 & 6.1 & 5.1 & 0.6 \\
\hline Gill opening width, 3rd & 7.5 & 1.8 & 4.2 & 1.7 & 1.2 & 2.2 & 1.6 & 0.3 \\
\hline Distance between first gill openings & 48.7 & 11.7 & 34.7 & 14.0 & 10.9 & 12.5 & 11.8 & 0.9 \\
\hline Distance between fifth gill openings & 34.7 & 8.3 & 24.1 & 9.7 & 8.0 & 8.9 & 8.5 & 0.5 \\
\hline Pelvic fin, length & 69.6 & 16.7 & 40.7 & 16.4 & 14.6 & 17.7 & 16.8 & 0.9 \\
\hline Pelvic fin, anterior margin length & 33.3 & 8.0 & 17.9 & 7.2 & 7.1 & 9.5 & 8.0 & 0.8 \\
\hline Pelvic fin, width & 30.1 & 7.2 & 18.6 & 7.5 & 5.8 & 7.6 & 6.8 & 0.5 \\
\hline Pelvic fin, base length & 35.1 & 8.4 & 21.7 & 8.8 & 7.8 & 12.1 & 9.8 & 1.5 \\
\hline Pelvic fin, inner margin length & 38.6 & 9.3 & 21.8 & 8.8 & 5.7 & 9.9 & 7.8 & 1.3 \\
\hline First dorsal fin, length & 26.1 & 6.3 & 15.2 & 6.1 & 5.8 & 6.8 & 6.3 & 0.3 \\
\hline First dorsal fin, anterior margin length & 36.6 & 8.8 & 21.0 & 8.5 & 7.6 & 8.9 & 8.3 & 0.4 \\
\hline First dorsal fin, height & 28.6 & 6.9 & 15.8 & 6.4 & 5.8 & 8.2 & 7.1 & 0.7 \\
\hline First dorsal fin, base length & 16.9 & 4.1 & 10.0 & 4.0 & 4.1 & 5.1 & 4.4 & 0.3 \\
\hline First dorsal fin, inner margin length & 9.8 & 2.4 & 5.4 & 2.2 & 1.5 & 2.7 & 2.1 & 0.3 \\
\hline Second dorsal fin, length & 26.8 & 6.4 & 15.7 & 6.3 & 6.0 & 7.6 & 6.5 & 0.4 \\
\hline Second dorsal fin, anterior margin length & 37.4 & 9.0 & 21.9 & 8.8 & 1.9 & 9.2 & 8.0 & 2.0 \\
\hline Second dorsal fin, height & 25.9 & 6.2 & 16.9 & 6.8 & 6.1 & 8.3 & 6.8 & 0.6 \\
\hline Second dorsal fin, base length & 17.5 & 4.2 & 10.9 & 4.4 & 4.1 & 5.4 & 4.6 & 0.4 \\
\hline Second dorsal fin, inner margin length & 9.6 & 2.3 & 6.1 & 2.5 & 1.4 & 2.7 & 2.1 & 0.4 \\
\hline
\end{tabular}


Table 3 (continued)

\begin{tabular}{|c|c|c|c|c|c|c|c|c|}
\hline & \multicolumn{2}{|c|}{$\begin{array}{l}\text { Acroteriobatus } \\
\text { leucospilus, } \\
\text { subadult male } \\
\text { syntype, BMNH } \\
\text { 1905.6.8.12 }\end{array}$} & \multicolumn{2}{|c|}{$\begin{array}{l}\text { Acroteriobatus } \\
\text { leucospilus, } \\
\text { juvenile male } \\
\text { syntype, BMNH } \\
1920.7 .23 .1\end{array}$} & \multirow{2}{*}{$\begin{array}{l}\text { Minimum } \\
(n=9)\end{array}$} & \multirow{2}{*}{$\begin{array}{l}\text { Maximum } \\
(n=9)\end{array}$} & \multirow{2}{*}{$\begin{array}{l}\begin{array}{l}\text { Mean } \\
(n=11)\end{array} \\
\% \mathrm{TL}\end{array}$} & \multirow[t]{2}{*}{ SD } \\
\hline & $\mathrm{mm}$ & $\% \mathrm{TL}$ & $\mathrm{mm}$ & $\% \mathrm{TL}$ & & & & \\
\hline Caudal fin, dorsal margin & 57.7 & 13.9 & 31.8 & 12.8 & 9.2 & 13.9 & 12.1 & 1.4 \\
\hline Caudal fin, preventral margin & 34.7 & 8.3 & 16.0 & 6.5 & 6.5 & 9.0 & 7.7 & 0.9 \\
\hline Snout to pelvic-fin origin ptp & 149.5 & 35.9 & 92.9 & 37.5 & 33.9 & 39.5 & 36.7 & 1.5 \\
\hline Snout to anterior vent & 160.0 & 38.5 & 100.1 & 40.4 & 38.2 & 42.6 & 39.7 & 1.2 \\
\hline Snout to first dorsal-fin origin & 245.0 & 58.9 & 146.8 & 59.2 & 58.8 & 61.4 & 59.8 & 0.9 \\
\hline Snout to second dorsal-fin origin & 309.2 & 74.3 & 185.1 & 74.6 & 74.4 & 77.1 & 75.5 & 0.9 \\
\hline Snout to upper caudal-fin origin & 356.6 & 85.7 & 212.7 & 85.8 & 83.0 & 89.4 & 86.7 & 1.8 \\
\hline Snout to lower caudal-fin origin & 363.0 & 87.3 & 217.0 & 87.5 & 86.5 & 91.8 & 88.7 & 1.6 \\
\hline Pelvic-fin insertion to dorsal-fin origin & 66.3 & 15.9 & 30.1 & 12.1 & 13.0 & 23.9 & 16.0 & 3.8 \\
\hline Interdorsal distance & 47.1 & 11.3 & 28.9 & 11.7 & 9.9 & 13.4 & 11.6 & 1.0 \\
\hline Disc width, anterior orbit & 79.7 & 19.2 & 53.2 & 21.5 & 16.9 & 23.8 & 19.5 & 2.2 \\
\hline Body width, pelvic insertion & 44.0 & 10.6 & 25.4 & 10.2 & 9.8 & 11.7 & 10.7 & 0.5 \\
\hline Body width, first dorsal-fin origin & 43.3 & 10.4 & 22.8 & 9.2 & 9.0 & 11.2 & 10.1 & 0.8 \\
\hline Body width, second dorsal-fin origin & 21.7 & 5.2 & 11.1 & 4.5 & 4.2 & 5.4 & 4.7 & 0.4 \\
\hline Body depth, maximum & 15.5 & 3.7 & 11.9 & 4.8 & 4.8 & 6.5 & 5.2 & 0.7 \\
\hline Body depth, pelvic-fin insertion & 18.2 & 4.4 & 10.4 & 4.2 & 4.1 & 5.2 & 4.5 & 0.3 \\
\hline Body depth, first dorsal-fin origin & 13.3 & 3.2 & 7.7 & 3.1 & 3.4 & 4.2 & 3.6 & 0.3 \\
\hline Body depth, second dorsal-fin origin & 8.9 & 2.1 & 5.3 & 2.1 & 2.0 & 2.7 & 2.3 & 0.2 \\
\hline Caudal peduncle length $=$ dorsal $(\mathrm{D} 2)$ - caudal space & 28.8 & 6.9 & 17.0 & 6.9 & 6.1 & 8.2 & 7.1 & 0.6 \\
\hline Tail length from anterior vent & 256.0 & 61.5 & 147.9 & 59.6 & 57.4 & 61.8 & 60.3 & 1.2 \\
\hline Angle before eyes, ${ }^{\circ}$ & \multicolumn{2}{|c|}{68.0} & \multicolumn{2}{|c|}{75.0} & 70.6 & 80.5 & 74.4 & 3.7 \\
\hline
\end{tabular}

Individual values for the subadult male syntype (BMNH 1905.6.8.12) and the juvenile male syntype (BMNH 1920.7.23.1), ranges for all examined nontype specimens $(n=9)$, as well as means and standard deviations $(\mathrm{SD})$ for all 11 examined specimens are presented. Proportional values are expressed as percentages of total length (TL) 70\% ethanol preserved except for minimum, maximum, and mean of TL in mm

distance across nostrils anteriorly, 2.63-9.5 in minimum internarial distance; process of flap about twice as long as wide, narrowing distally to blunt pointed tip, and adjoining with posterolateral nasal flap and anterior aperture posterior margin. Posterolateral nasal flap well developed, lobe-like, broadest medially, length $2.0-5.5$ times its width; originating just behind lateral extremity of anterior nasal aperture, extending posteromesially as a free fold almost to the level of insertion of posterior nasal flap. Posterior nasal flap well developed, lobe-like, base length 1.6-2.5 times its width, its inner edge nearly reaching innermost margin of nostril; width 0.5 1.0 times anterior aperture width, $0.92-2.0$ times posterolateral nasal-flap width (Figs. 34 and 35).

Mouth width 1.59-2.24 times nostril length, 5.94-7.25 in precloacal length; positioned beneath posterior margin of orbit. Upper jaw slightly convex, upper lip broadly arched; lower lip not pronounced, not separated from oral groove by ridges of strongly corrugated skin; corners of mouth with several strong, short lateral grooves. Teeth arranged in quincunx, small, close-set, rhombic, broad-based with short, bluntly rounded cusp; upper and lower teeth similar in shape and size; upper and lower jaws each with $\sim 60-75$ tooth rows. Gill openings strongly wavy; length of third gill slit 1.63-3.4 in nostril length, 4.33-7.0 in distance between fifth gill slits; distance between first gill slits 1.31-1.46 times distance between fifth gill slits; distance between fifth gill slits 2.54-3.24 times internarial distance, 1.3-1.62 times mouth width, 0.32 0.38 of ventral head length (Fig. 35).

Dorsal fins erect, relatively tall, dissimilar in size and shape; first dorsal-fin anterior margin weakly convex, curving rearwards towards rounded apex; posterior margin weakly convex near tip, then becoming weakly concave to nearly straight; second dorsal-fin anterior margin weakly convex, curving towards pointed or slightly rounded apex; posterior margin nearly straight; free rear tips rounded of both dorsal fins, almost forming right angle, not produced; first dorsal fin 
height subequal to second, length of first $0.79-1.15$ times height, its base length 1.67-2.8 times inner margin length; second dorsal-fin length $0.84-1.21$ times its height, base length 1.5-3.3 times inner margin length (Fig. 36a, b). First dorsal-fin origin well posterior to pelvic-fin free rear tips, interspace 1.04-1.99 times interdorsal distance; interdorsal space relatively short, 1.32-2.19 times second dorsal-fin height, 2.13-3.17 times base of first dorsal fin, 0.98-1.33 times tail width at origin of first dorsal fin, 1.29-1.9 times interspace between second dorsal-fin insertion and upper origin of caudal fin. Caudal fin relatively small; dorsal caudal margin weakly convex, length 1.03-1.99 times preventral margin length; ventral lobe broadly rounded; posterior margin weakly concave; fin tip acutely pointed (Fig. 36c).

Dermal denticles mostly minute, close-set, covering entire body and fins; surfaces mostly smooth to the touch, except for $\sim 7$ slightly enlarged granular denticles on each orbital rim and $\sim 3-4$ on the inner edge of spiracle; up to $\sim 50$ enlarged, coarse denticles rather regularly distributed along midline from nape to near first dorsal-fin origin, absent along midline between dorsal fins and upper caudal origin; enlarged denticles absent on snout tip, anterior and lateral margins of disc, pelvic fins, and bases of dorsal fins; nasal flaps and lamellae naked. Crowns of denticles mostly oval, except largest denticles with slightly erect, bluntly acute cusp, base stellate. Juveniles have proportionally larger denticles than larger specimens and the crowns of the denticles have acutely pointed cusps. The minute dermal denticles covering entire body and fins are also proportionally in juveniles, giving the skin a velvet touch. Thorns always absent. Prebranchial sensory pore patch distinct, extending to about the level of third gill slit. Postscapular sensory canal rather indistinct, strongly undulated anteriorly, terminating near pectoral-fin insertions; sensory pores minute, canal not forming a shallow groove (Fig. 29).

Rostral cartilage broad, its shaft just slightly increasing in width posteriorly from rostral node; rostral node rounded at apex, not angular, broadly expanded, relatively short, axis at maximum node width $34.4-41.2 \%$ of length of rostral cartilage from tip; anterior fontanelle relatively broad posteriorly, tapering gradually towards rostral node, dorsolateral edges of cartilage surrounding fontanelle (rostral ridges on surface of snout) well separated posteriorly, not constricting medially; rostral cartilage $54.2-58.1 \%$ of neurocranium length, ventral edges of rostral cartilage united; nasal capsules large, with their transverse axes directed anterolaterally; width of cranium across nasal capsules 1.15-1.27 times nasobasal length (base of rostrum to occipital condyles); width of nasal capsule 1.31.45 times its length; basal plate relatively broad, its minimum width 3.35-3.61 times in nasobasal length; cranial roof with small, oval-shaped fenestra, with its anterior edge located behind precerebral cavity by a distance $\sim 1.5$ times its length; anterior cartilage triangular, narrow, posteriorly wedgeshaped, without an anterior lobe extending past nasal capsules; preorbital processes well developed; postorbital processes moderately large, not bifurcate; greatest width across processes 1.78-1.97 times in nasobasal length (Fig. 37).

Nasal lamellae 37-41. Skeletal meristics based on the two syntypes and non-type specimens SAIAB 189087, SAIAB 34588, and SAIAB 55691: pectoral skeleton with 29-32 propterygial, 8-9 mesopterygial, 1-2 neopterygial, 22-27 metapterygial, amounting to $63-70$ total radials. Total pelvic-fin radials 25-30. Total vertebral segment (synarcual and free) counts 200-205; post-synarcual centra 187-192; precaudal centra (excluding synarcual centra) 148-151; synarcual segments $13-15$; monospondylous precaudal centra 39-41; diplosondylous precaudal centra 109-110; diplosondylous caudal centra 37-42. Monospondylous to diplosondylous centra transition posterior to pelvic girdle.

\section{Coloration}

Prior to preservation: dorsal surface sandy brown with a pronounced and colorful, organized pattern of numerous small and large bluish circular spots, with a darker ring encircling a lighter bluish center, and small and large brownish spots (Fig. 31); the bluish spots cover the snout, pectoral, pelvic, dorsal, and caudal fins but not the central disc; the brownish spots cover the disc, tail, fin bases, and fins, sometimes giving the caudal peduncle a striped appearance (Fig. 31); snout semi-translucent with elongated bluish spots giving it a stripe-nosed appearance (Fig. 31). Outer edges of pectoraland pelvic-fin margins blue, lateral tail folds white or striped blue and brown (Fig. 31). Ventral surface white. After preservation: dorsal surface similar but bluish spots less pronounced and brownish spots mostly faded and not evident (Figs. 28 and 30). Ventral surface also similar but creamy rather than white as typical for preserved specimens (Fig. 29).

\section{Size}

The 11 examined specimens ranged in size from 223 to $960 \mathrm{~mm}$ TL. Acroteriobatus leucospilus has been reported to reach 1200 mm TL (Compagno et al. 1989; Séret et al. 2016; Weigmann 2016), but no voucher specimens of such size are known and the maximum size might possibly be based on the larger A. andysabini sp. nov. instead. The largest verified specimen is the adult female of $960 \mathrm{~mm}$ TL examined in the present study. The $416 \mathrm{~mm}$ TL male syntype BMNH 1905.6.8.12 and the $430 \mathrm{~mm}$ TL non-type male SAIAB 40000 are both subadult, and the $690 \mathrm{~mm}$ TL non-type female SAIAB 34588 is gravid. Following Wallace (1967), a male of $402 \mathrm{~mm}$ TL was juvenile, one of $415 \mathrm{~mm}$ TL was subadult, 
and specimens of more than $565 \mathrm{~mm}$ TL were mature, with gravid females ranging from 564 to $921 \mathrm{~mm}$ TL. Considering the rather small maturing size of about $415-565 \mathrm{~mm} \mathrm{TL}$, a maximum size above $1000 \mathrm{~mm}$ TL appears unlikely. Wallace (1967) indicates a size of $93 \mathrm{~mm}$ TL and a weight of $1 / 4 \mathrm{lb}$ for the smallest embryo seen and $250 \mathrm{~mm}$ TL for the smallest juvenile on record (allegedly also weighing 1/4 lb). Based on the maximum verified size of $960 \mathrm{~mm}$ TL, A. leucospilus is a medium-sized species of the genus.

\section{Distribution}

Acroteriobatus leucospilus so far has been recorded from off the central Transkei coast (Eastern Cape Province) to the northern KwaZulu-Natal Province (South Africa) and Mozambique. Ebert et al. (2021), based on the examined material, extended its range to Zanzibar and Dar es Salaam, Tanzania (Fig. 13). It is known from 0 to $100 \mathrm{~m}$ depth (Compagno et al. 1989; Séret et al. 2016; Weigmann 2016) but seems to be most abundant in depths of around 20 fathoms or $37 \mathrm{~m}$ (Wallace 1967).

\section{Discussion}

The genus Acroteriobatus can be subdivided into two subgroups: those with elongated bluish-gray spots on the dorsal snout surface giving it a stripe-nosed color pattern and those without. The six species with stripe-nosed pattern include A. andysabini sp. nov., A. stehmanni sp. nov., A. leucospilus, Acroteriobatus salalah (Randall \& Compagno, 1995), Acroteriobatus variegatus (Nair \& Lal Mohan, 1973), and A. zanzibarensis. Among the stripenosed subgroup, only $A$. andysabini sp. nov. and A. variegatus have black blotches or spots on the ventral snout surface at all sizes. Acroteriobatus salalah and the poorly known A. zanzibarensis sometimes have dark blotches or spots on the underside of the snout, but not always. The other two species (A. stehmanni sp. nov. and A. leucospilus) have a plain white ventral snout color (except for the smallest juvenile paratype ZMH 25560 of $A$. stehmanni sp. nov.). The four species of the other subgroup (Acroteriobatus annulatus (Smith in Müller \& Henle, 1841), Acroteriobatus blochii (Müller \& Henle, 1841), Acroteriobatus ocellatus (Norman, 1926), and Acroteriobatus omanensis Last, Henderson \& Naylor, 2016b) all lack a stripe-nosed pattern on the dorsal snout surface and have a plain light to white ventral snout surface without a dark blotch.

Acroteriobatus andysabini sp. nov. can be separated from all other Acroteriobatus species by a combination of characters including black spots on the ventral surface of its snout at all sizes and in both sexes. All other known members of the genus, except for A. variegatus, are either plain white or variably may have a dark blotch on the snout tip depending on the size. Its closest geographic congener, A. leucospilus, is plain white on the ventral snout surface at all sizes from neonates to adults, while in $A$. andysabini sp. nov., the black spots become more dense with size. Furthermore, A. andysabini sp. nov. has a higher nasal lamellae count than A. leucospilus (42-48 vs. $37-41)$, more obtuse snout angle ( $76-85^{\circ}$ vs. $\left.68-81^{\circ}\right)$, bluishgray but no dark brown spots dorsally on central disc, outer edges of pelvic-fin margins white, lateral tail folds striped orange and white (vs. no bluish-gray but dark brown spots dorsally on central disc, outer edges of pectoral- and pelvicfin margins blue, lateral tail folds white or striped blue and brown), and larger maturity (above $603 \mathrm{~mm}$ vs. 415-565 mm TL) and maximum (more than $1000 \mathrm{~mm}$ vs. $960 \mathrm{~mm}$ TL) sizes. In addition, the anterior disc margin is weakly concave anteriorly, becoming convex posterior to constriction at the level of first propterygials, turning to weakly concave posterior to the level of posterior margin of spiracles and again becoming weakly convex towards broadly rounded apex (vs. straight); there is a continuous row of $\sim 15-20$ somewhat enlarged granular denticles around the anterior and inner rim of orbits, extending to the anterior edge of inner spiracle (vs. $\sim 7$ denticles on each orbital rim and $\sim 3-4$ on the inner edge of spiracle), and the nasal capsules are proportionally wider (width $1.56-1.87$ vs. 1.3-1.45 times length). Geographically, A. andysabini sp. nov. appears to be the only species of the genus occurring off Madagascar, where it is apparently endemic. Acroteriobatus leucospilus is distributed along the eastern African coast from KwaZulu-Natal Province (South Africa) to Zanzibar, Tanzania. Acroteriobatus andysabini sp. nov. differs from $A$. salalah particularly in the shape of the snout and disc (snout moderately long and narrowly angular anteriorly, snout angle before eyes moderately acute, $76-85^{\circ}$, disc wedge-shaped with undulate anterior margin vs. snout short and obtusely angled at $\sim 88^{\circ}$, disc heart-shaped with convex anterior margins) and size (maximum size $>1000 \mathrm{~mm}$ vs. $780 \mathrm{~mm}$ TL). Compared to $A$. variegatus and A. zanzibarensis, this new species grows larger (maximum size $>1000 \mathrm{~mm}$ vs. $750-790 \mathrm{~mm}$ TL), has a less acutely angled $\left(76-85^{\circ}\right.$ vs. $\left.59-64^{\circ}\right)$ snout without (vs. with) orange stripes, and shows a pronounced pattern of bluish-gray spots on disc, tail, and fins (vs. only few bluish-gray spots on disc, tail, and fins).

Acroteriobatus stehmanni sp. nov. can be separated from all other Acroteriobatus species by a combination of characters including the patterns of bluish-gray and brown spots on the dorsal surface. In contrast to all other members of the stripe-nosed subgroup of Acroteriobatus, A. stehmanni sp. nov. only has a few, somewhat elongated bluish-gray spots, 
giving it a very reduced stripe-nosed appearance, a generally sparse patterning with small bluish-gray circular spots, confined to symmetrical patterns on snout tip, posterior pectoralfin margins, a pair of tiny spots on midbody behind occipital joint, and few spots on posterior pelvic-fin margins. The brown spots on the body and dorsal and caudal fins are indistinct, and the ventral surface is white except for a blackish blotch and two tiny black spots on the ventral snout tip in the smallest juvenile paratype. Compared to its morphologically closest congener, A. leucospilus, A. stehmanni sp. nov. also has a higher nasal lamellae count (43-48 vs. 37-41), smaller maturity (starting to mature at $\sim 378$ vs. maturing between 415 and $565 \mathrm{~mm} \mathrm{TL}$ ), and maximum (622 mm vs. $960 \mathrm{~mm}$ TL) sizes, a shorter dorsal head length (17.2-22.8\% vs. $24.0-29.2 \%$ TL), preoral length $2.63-3.02$ vs. $2.22-2.76$ times mouth width and 5.46-6.9 vs. 4.28-5.08 times internarial distance, direct preorbital snout length 2.59-2.78 vs. 2.17-2.6 times interspiracular distance and 3.83-4.32 vs. 2.65-3.67 times skeletal interorbital space, post-scapular sensory canal distinct and weakly undulated anteriorly vs. rather indistinct and strongly undulated anteriorly, and nasal capsules proportionally wider (width $1.39-1.68$ vs. $1.3-1.45$ times length). Furthermore, A. leucospilus seems to be allopatric, distributed along the eastern African coast from KwaZulu-Natal Province (South Africa) to Zanzibar, Tanzania. Geographically, A. stehmanni sp. nov. appears to be sympatric only with $A$. salalah, which has very recently been confirmed for the Socotra Islands (Bogorodsky et al. 2021). Acroteriobatus stehmanni sp. nov. can easily be distinguished from $A$. salalah by the shape of the snout and disc (snout moderately long and narrowly angular anteriorly, snout angle before eyes moderately acute, $71-77^{\circ}$, disc wedgeshaped with straight anterior margin vs. snout short and obtusely angled at $\sim 88^{\circ}$, disc heart-shaped with convex anterior margins) and dorsal color pattern (snout with only a few, somewhat elongated bluish-gray spots, giving it a very reduced stripe-nosed appearance vs. snout usually with pronounced stripe-nosed pattern). Compared to A. variegatus and $A$. zanzibarensis, this new species is smaller (maximum size $622 \mathrm{~mm}$ vs. $750-790 \mathrm{~mm}$ TL, maturing starts at $378 \mathrm{~mm}$ TL vs. maturity size 580-640 $\mathrm{mm}$ TL) and has a less acutely angled $\left(71-77^{\circ}\right.$ vs. $\left.59-64^{\circ}\right)$ snout without (vs. with) orange stripes and without (vs. with) pronounced stripe-nosed pattern.

Differences between both new species (A. andysabini sp. nov. and A. stehmanni sp. nov.) include the dorsal color pattern (bluish-gray but no dark brown spots dorsally on central disc, outer edges of pelvic-fin margins white, lateral tail folds striped orange and white in A. andysabini sp. nov. vs. very reduced stripe-nosed pattern and generally sparse patterning with small bluish-gray circular spots in A. stehmanni sp. nov.), snout angle $\left(76-85^{\circ}\right.$ vs. $\left.71-77^{\circ}\right)$, shape of anterior disc margin (weakly concave anteriorly, becoming convex posterior to constriction at the level of first propterygials, turning to weakly concave posterior to the level of posterior margin of spiracles and again becoming weakly convex towards broadly rounded apex vs. straight), dorsal head length (24.2-33.5\% vs. 17.2-22.8\% TL), ventral coloration (blacks spots on the ventral snout at all sizes and in both sexes vs. ventral snout white except for a large blotch and two tiny spots in smallest juvenile paratype), as well as maturity (above 603 vs. starting to mature at $\sim 378 \mathrm{~mm} \mathrm{TL}$ ) and maximum (more than $1000 \mathrm{~mm}$ vs. $622 \mathrm{~mm}$ TL) sizes. Additionally, A. andysabini sp. nov. has a continuous row of $\sim 15-20$ somewhat enlarged granular denticles around the anterior and inner rim of orbits, extending to the anterior edge of inner spiracle vs. 4-5 slightly enlarged granular denticles on each anterior orbital rim, 1-2 on each inner posterior rim, and 2 on the inner edge of each spiracle in A. stehmanni sp. nov. and the post-scapular sensory canal is rather indistinct vs. distinct. Furthermore, the preoral length is 2.0-2.62 vs. 2.63-3.02 times mouth width and 3.754.89 vs. 5.46-6.9 times internarial distance, the direct preorbital snout length is $1.92-2.39$ vs. $2.59-2.78$ times interspiracular distance and 3.09-3.63 vs. 3.83-4.32 times skeletal interorbital space, the distance between bases of spiracular folds is $0.3-0.7$ vs. $0.85-1.83$ times length of shortest fold, and the second dorsal-fin base length is 1.34-2.21 vs. 2.01-3.5 times inner margin length.

Biogeographically, Acroteriobatus andysabini sp. nov. appears to be endemic to Madagascar, while its closest geographic congener, A. leucospilus, appears to occur from off the central Transkei coast (Eastern Cape Province), South Africa, to at least Zanzibar, Tanzania. Literature accounts only list A. leucospilus as occurring off South Africa and Mozambique (Compagno et al. 1989; Séret et al. 2016), but here, we can confirm its distribution further north. Recently, A. leucospilus was reported from around Madagascar for the first time (Fricke et al. 2018), but that record, as well as subsequent records by Ghilardi et al. (2019), are now referable to A. andysabini sp. nov. Acroteriobatus zanzibarensis is geographically close but presently only known from off Zanzibar and Kenya. The only other geographically close species are the common A. annulatus, which occurs off Namibia to South Africa, and the poorly known A. ocellatus, which is only known from a few specimens off the Eastern Cape Province near Algoa Bay, South Africa (Compagno et al. 1989; Séret et al. 2016; Ebert et al. 2021). Four other species (A. omanensis, A. salalah, A. stehmanni sp. nov., and A. variegatus) are only known from the northern Indian Ocean, and all have very limited distributions (Séret et al. 
2016; present study). The validity of $A$. variegatus and its separation from $A$. zanzibarensis are currently being reassessed, as both species are possibly conspecific (unpublished data; see also Séret et al. 2016). Acroteriobatus blochii occurs in the southeastern Atlantic off the west coast of South Africa to Namibia (Compagno et al. 1989; Ebert et al. 2021).

\section{Conservation implications}

Shark-like rays, specifically those of the order Rhinopristiformes, are among the most threatened species of cartilaginous fish globally (Dulvy et al. 2014; Kyne et al. 2020). This is due to life history characteristics such as slow growth, late age at maturity, and low fecundity, combined with a mostly nearshore coastal ( $<100 \mathrm{~m}$ depth) habitat that makes them vulnerable to overfishing and habitat degradation. The sawfishes (Pristidae) are perhaps the best-known family but also are one the most Critically Endangered groups, with all species having been extirpated from much of their ranges (Dulvy et al. 2016; Yan et al. 2021). More recently, the wedgefishes (Rhinidae) and giant guitarfishes (Glaucostegidae) have come into the public conscious as 15 of 16 species were assessed as being Endangered or Critically Endangered based on the International Union for Conservation of Nature (IUCN) Red List of Threatened Species categories (Kyne et al. 2020). While steps have been taken to address concerns over these better known rhinopristiform batoids, the small-bodied guitarfishes (Rhinobatidae) have mostly been overlooked even though they share the same life history and habitat characteristics, and are subject to intense fishing pressure and habitat degradation (Moore 2017; Jabado 2018; Jabado et al. 2018). Further compounding conservation and fisheries management efforts have been lack of taxonomic clarity and speciesspecific identification.

The implications for conservation and management concerns surrounding the two newly described Acroteriobatus species are considerable. First, the small-scale fishery for guitarfish in Madagascar (Fig. 12) has increased, coinciding with an increased demand for shark fins, including guitarfishes, in Asia (Humber et al. 2017). In fact, guitarfishes were one of three most important species and accounted for $75 \%$ of the overall elasmobranch landings in Madagascar (Humber et al. 2017). Two species of guitarfish are now known to occur off Madagascar: the new species $A$. andysabini sp. nov. and the recently described Rhinobatos austini Ebert \& Gon, 2017. However, these two species are lumped into a single category, i.e., guitarfishes, without species-specific information, which is fundamental to effective conservation and management policy (Moore 2017). Presently, there are no management or conservation measures in place for sharks and rays in Madagascar (Humber et al. 2017). This is of significant concern since the distribution of $A$. andysabini sp. nov. seems to be restricted to Madagascar. Second, the distribution of A. leucospilus is much more restrictive than previously thought; it now appears to only occur on the east coast of South Africa to Tanzania (present study; Ebert et al. 2021). Acroteriobatus leucospilus has been assessed as Endangered by the IUCN Red List of Threatened Species (Pollom et al. 2019), but with its distribution now being more restrictive than previously thought, management and conservation policies may need to be reviewed. Third, A. stehmanni sp. nov. presently appears to be restricted to the waters surrounding Socotra Islands and may be subject to intense traditional fisheries (Moore 2017; Jabado 2018; Jabado et al. 2018). Improvement for the situation of the two guitarfish species occurring off Madagascar might be achieved through the national plan of action for sharks and rays in Madagascar (Plan national de gestion et de conservation des requins et des raies à Madagascar, PNGCRR) (Anonymous 2019a), associated to an implementation plan for the period 2019-2023 (Anonymous 2019b).

Most Acroteriobatus species, including the two new species, appear to be endemic or have very restrictive distributions. Nine of 10 Acroteriobatus species occur in the western Indian Ocean (WIO), and all are subject to intense coastal fisheries, which are reflected in the IUCN Red List of Threatened Species (IUCN 2020); the southeast Atlantic A. blochii only occurs outside this region. The conservation status of WIO Acroteriobatus guitarfishes reveals that three species are threatened, including $A$. variegatus (Critically Endangered), A. leucospilus (Endangered), and A. annulatus (Vulnerable), and A. salalah and A. zanzibarensis are Near Threatened. Two species (A. ocellatus and A. omanensis) are so poorly known they were assessed as Data Deficient, yet they occur in coastal areas subject to intense fisheries.

The recognition of two new species (A. andysabini sp. nov. and $A$. stehmanni sp. nov.), with the redescription of A. leucospilus, clarifies the taxonomic status and distribution of these three small-bodied guitarfishes. This is essential for improved data collection and research and for more effective conservation and management policy decisions. 


\section{Key to the 10 valid species of Acroteriobatus}

1 Snout dorsally with elongated bluish-gray spots, giving it a stripe-nosed color pattern (6 species) 2

- Snout dorsally lacking a stripe-nosed pattern, ventrally plain light to white without a dark blotch (4 species) ........................... 7

2 Snout short and obtusely angled $\left(\sim 88^{\circ}\right)$, disc heart-shaped with convex anterior margins A. salalah (NW Indian Ocean: Socotra Islands to Pakistan)

- Snout longer and narrowly pointed, disc wedge-shaped with nearly straight to slightly concave anterior margins 3

3 Snout with only a few, somewhat elongated bluish-gray spots, giving it a very reduced stripe-nosed appearance, maximum size of $622 \mathrm{~mm}$ TL A. stehmanni sp. nov. (NW Indian Ocean: Socotra Islands)

- Snout with pronounced stripe-nosed pattern, maximum size of $750 \mathrm{~mm}$ TL or larger .

4 Snout with orange stripes between the elongated bluish-gray spots, snout acutely angled at $59-64^{\circ}$, only few bluish-gray spots on disc, tail, and fins; maximum size of 750-790 mm TL

- Snout without orange stripes, snout less acutely angled at $68-85^{\circ}$, pronounced pattern of bluish-gray spots on disc, tail, and fins; maximum size of $960 \mathrm{~mm}$ TL or larger....

5 Dorsal surface greenish brown with numerous large dark brown blotches all over the dorsal surface; ventral surface may be covered with small dark spots A. zanzibarensis (NW Indian Ocean: Zanzibar, Kenya)

- Dorsal surface sandy brown with a strong orangish touch, particularly on snout, and without distinct dark blotches; no specimens with dark ventral spots apart from underside of snout known A. variegatus (N Indian Ocean: India, Sri Lanka)

6 Snout angle $79-85^{\circ}$, nasal lamellae $45-48$, bluish-gray but no dark brown spots dorsally on central disc, outer edges of pelvicfin margins white, lateral tail folds striped orange and white, ventral snout with black blotches or spots, maturing size above $602 \mathrm{~mm}$ TL, maximum size of more than $1000 \mathrm{~mm}$ TL ............... A. andysabini sp. nov. (W Indian Ocean: Madagascar) - Snout angle $68-81^{\circ}$, nasal lamellae 37-41, no bluish-gray but dark brown spots dorsally on central disc, outer edges of pectoraland pelvic-fin margins blue, lateral tail folds white or striped blue and brown, ventral surface plain white, maturing size of 415-565 mm TL, maximum size of $960 \mathrm{~cm} \mathrm{TL}$.................. A. leucospilus (W Indian Ocean: South Africa to Tanzania) 7 Disc shovel-shaped, snout obtusely angled at $\sim 95^{\circ}$, posterior margin of each spiracle with only one dermal fold A. blochii (SE Atlantic Ocean: South Africa to Namibia)

- Disc wedge-shaped, snout acutely angled at $\sim 60-70^{\circ}$, each spiracle with two dermal folds on posterior margin ....................... 8

8 Dorsal surface brownish with numerous small symmetrically arranged ocelli consisting of a white center surrounded by a darker brown rim, snout tip very narrowly pointed, disc narrow (length 1.3-1.4 times width) ........ A. omanensis (NW Indian Ocean: Gulf of Oman) - Dorsal surface with dark spots or small ocelli, but each ocellus either consisting of a small central dark spot surrounded by a dark-edged pale ring or with bluish-gray center surrounded by a dark brown ring, snout tip less narrowly pointed, disc relatively broad (length $\sim 1.1$ times width)

9 Dorsal surface light brown with a more or less symmetrical pattern of either dark spots (Natal area) or small ocelli, each consisting of a small central dark spot surrounded by a dark-edged pale ring (South African Cape Province) A. annulatus (SE Atlantic and W Indian oceans: South Africa) - Dorsal surface brownish with numerous irregularly shaped ocelli with bluish-gray centers surrounded by a dark brown rim A. ocellatus (SW Indian Ocean: South Africa)

\begin{abstract}
Acknowledgements The authors are very grateful to the following individuals, institutions, and organizations for their assistance and support: Ralf Thiel $(\mathrm{ZMH})$ for granting access to and loan of specimens in the $\mathrm{ZMH}$ collection and access to radiography facilities at $\mathrm{ZMH}$; Irina Eidus $(\mathrm{ZMH})$ for her help with the radiography and collection database; Matthias F.W. Stehmann (ICHTHYS), who collected all type specimens of Acroteriobatus stehmanni sp. nov., kindly provided fresh photographs of two of them, and granted permission to use them in the present paper; Frederik Mollen (Elasmobranch Research Belgium) who kindly sent on loan to SW the three ERB specimens of A. zanzibarensis and provided tissue samples; Aaron Henderson (United Arab Emirates University) and Uwe Zajonz (SMF) who kindly provided numerous photographs of A. salalah specimens; and Radford Arrindell (AMNH), Leonid G. Manilo (HMNH NASU), and Sandra Raredon (USNM), who kindly provided collection numbers, total length measurements, and
\end{abstract}

photographs of paratypes under their care. Elaine Heemstra (SAIAB), Kerry Sink (SANBI), Marsha Englebrecht, and Dennis King kindly provided images and granted permission to use them in the present paper. James Maclaine (BMNH) kindly took radiographs (reworked by Nemo Martin) and arranged photographs, taken by Kevin Webb, of the syntypes of A. leucospilus, and granted permission to use them in the present paper. Dave Catania and Jon Fong (CAS) provided collection support and radiographs for the holotype and eight paratypes of A. andysabini sp. nov. The holotype of $A$. andysabini sp. nov. at SAIAB was made possible by the work of Philippe Bouchet, the principal investigator and organizer of the Atimo Vatae expedition, a joint venture of the Muséum national d'Histoire naturelle (MNHN) and Pro-Natura International, together with the Institut d'Halieutique Sciences Marines, University of Toliara, and the Wildlife Conservation Society, Madagascar Programme. Bouchet also arranged for the donation of specimens to the South African Institute 
for Aquatic Biodiversity (NRF-SAIAB). General assistance and support was kindly provided by Angus Paterson, Paul Cowley, Elaine Heemstra, Phil Heemstra, Roger Bills, Mzwandile Dwani, Nkoshinathi Mazungula, Vuyani Hanisi, and the fish collection staff (SAIAB), Rhett Bennett, Mike Markovina, Abdalla Said Abdulla, Naseeba Sidat, Jorge Sitoe, and Eleuterio Duarte (Wildlife Conservation Society); Stela Fernando (Instituto de Investigação Pesqueira, Mozambique); Baraka Kuguru (Tanzania Fisheries Research Institute); Marsha Englebrecht; Andy Sabin; Moss Landing Marine Laboratories; and the Save Our Seas Foundation, South African Shark and Ray Protection Project, implemented by the WILDTRUST, funded by the Shark Conservation Fund and the South African Institute for Aquatic Biodiversity. The authors are grateful to journal editors and reviewers of the manuscript for their helpful comments.

Funding Open Access funding enabled and organized by Projekt DEAL. This project was supported in part by the Save Our Seas Foundation, South African Shark and Ray Protection Project, implemented by the WILDTRUST, funded by the Shark Conservation Fund and the South African Institute for Aquatic Biodiversity.

\section{Declarations}

Conflict of interest The authors declare that they have no conflict of interest.

Ethical approval All applicable international, national, and/or institutional guidelines for animal testing, animal care, and use of animals were followed by the authors.

Sampling and field studies All necessary permits for sampling and observational field studies have been obtained by the authors from the competent authorities and are mentioned in the acknowledgements, if applicable. The study is compliant with CBD and Nagoya protocols.

Data availability All data generated or analyzed during this study are included in this published article.

Author contribution SW and DAE conceived and designed the research. SW, DAE, and BS conducted the examinations. SW and DAE wrote the initial version of the manuscript. All authors read and approved the final manuscript.

Open Access This article is licensed under a Creative Commons Attribution 4.0 International License, which permits use, sharing, adaptation, distribution and reproduction in any medium or format, as long as you give appropriate credit to the original author(s) and the source, provide a link to the Creative Commons licence, and indicate if changes were made. The images or other third party material in this article are included in the article's Creative Commons licence, unless indicated otherwise in a credit line to the material. If material is not included in the article's Creative Commons licence and your intended use is not permitted by statutory regulation or exceeds the permitted use, you will need to obtain permission directly from the copyright holder. To view a copy of this licence, visit http://creativecommons.org/licenses/by/4.0/.

\section{References}

Amante C, Eakins BW (2009) ETOPO1 1 arc-minute global relief model: procedures, data sources and analysis. NOAA Tech Mem NESDIS NGDC-24, 19 pp
Anonymous (2019a) Plan National de Gestion et de Conservation des Requins et des Raies à Madagascar. 2019-2023. Ministère de l'Environnement, de l'Ecologie et des forêts (MEEF), Ministère de la Pêche et des Ressources Halieutiques et de la Pêche (MRHP), Repoblikan'I Madagasikara, $28 \mathrm{pp}$

Anonymous (2019b) Plan de mise en oeuvre du Plan National de Gestion et de Conservation des Requins et des Raies à Madagascar. 20192023. Ministère de l'Environnement, de l'Ecologie et des forêts (MEEF), Ministère des Ressources Halieutiques et de la Pêche (MRHP), Repoblikan'I Madagasikara, 20 pp

Barnard KH (1925) A monograph of the marine fishes of South Africa. Part I (Amphioxus, Cyclostomata, Elasmobranchii, and Teleostei Isospondyli to Heterosomata). Ann S Afr Mus 21(1):1-418

Barnard KH (1927) A monograph of the marine fishes of South Africa. Part II (Teleostei - Discocephali to end. Appendix). Ann S Afr Mus 21(1):419-1065

Bogorodsky SV, Zajonz U, Saeed FN, Weigmann S (2021) Notes on batoid fishes of the Socotra Archipelago (north-western Indian Ocean), with four new records. Zootaxa 4951(3):511-528. https:// doi.org/10.11646/zootaxa.4951.3.5

Bonaparte CL (1835) Prodromus systematis ichthyologiae. Nuovi Ann Sci nat Bologna (Ser 1) 2(4):181-196 272-277

Bouchet P (ed) (2014) « La Planète Revisitée » à Madagascar. Exploration et inventaire de la biodiversité marine à Madagascar. Bilan de l'expédition. MNHN, Pro-Natura International, Wildlife Conservation, Institut d'Halieutique et des Sciences Marines de Tuléar, $86 \mathrm{pp}$

Compagno LJV, Ebert DA, Smale MJ (1989) Guide to the sharks and rays of Southern Africa. New Holland Ltd., London 158 pp

Dulvy NK, Fowler SL, Musick JA, Cavanagh RD, Kyne PM, Harrison LR, Carlson JK, Davidson LNK, Fordham SV, Francis MP, Pollock CM, Simpfendorfer CA, Burgess GH, Carpenter KE, Compagno LJV, Ebert DA, Gibson C, Heupel MR, Livingstone SR, Sanciangco JC, Stevens JD, Valenti S, White WT (2014) Global extinction risk of sharks and rays: threats, hotspots, and conservation. eLife 2014 3:e00590. https://doi.org/10.7554/eLife.00590

Dulvy NK, Davidson LNK, Kyne PM, Simpfendorfer CA, Harrison LR, Carlson JK, Fordham SV (2016) Ghosts of the coast: global extinction risk and conservation of sawfishes. Aquat Conserv 26:134-153. https://doi.org/10.1002/aqc. 2525

Ebert DA, Gon O (2017) Rhinobatos austini n. sp., a new species of guitarfish (Rhinopristiformes: Rhinobatidae) from the southwestern Indian Ocean. Zootaxa 4276(2):204-214. https://doi.org/10.11646/ zootaxa.4276.2.3

Ebert DA, van Hees KE (2015) Beyond jaws: rediscovering the 'lost sharks' of southern Africa. Afr J Mar Sci 37(2):141-156. https:// doi.org/10.2989/1814232X.2015.1048730

Ebert DA, Wintner SP, Kyne PM (2021) An annotated checklist of the chondrichthyans of South Africa. Zootaxa 4947(1):1-127. https:// doi.org/10.11646/zootaxa.4947.1.1

Fourmanoir P (1963) Raies et requins-scie de la côte ouest de Madagascar (Ordre des Batoidei). Cah ORSTOM Océanogr 1963(6):33-58

Fowler HW (1941) Contributions to the biology of the Philippine archipelago and adjacent regions. The fishes of the groups Elasmocephalii, Holocephali, Isospondyli, and Ostariophysi obtained by the United States Fisheries Steamer "Albatross" in 1907 to 1910, chiefly in the Philippine islands and adjacent seas. Bull US Natl Mus 100:1-879

Fricke R, Mahafina J, Behivoke F, Jaonalison H, Léopold M, Ponton D (2018) Annotated checklist of the fishes of Madagascar, southwestern Indian Ocean, with 158 new records. FishTaxa 3(1):1-432

Ghilardi M, Emmanuel P, Gibbons E (2019) Guitarfish fishing in southwest Madagascar: an assessment of the status of grayspotted guitarfish (Acroteriobatus leucospilus). WIOMSA $11^{\text {th }}$ scientific symposium, July 1-6, 2019, University of Mauritius, Reduit 
Gilchrist JDF, Thompson WW (1911) Descriptions of fishes from the coast of Natal (part III). Ann S Afr Mus 11(2):29-58

Gilchrist JDF, Thompson WW (1916) A catalogue of the sea fishes recorded from Natal, part I. Ann Durban Mus 1(3):284-289

Giltay L (1928) Notes ichthyologiques: II. Une espèce nouvelle de Rhinobatus du Congo belge (Rhinobatus congolensis, nov. sp.). Ann Soc r Zool Bel 59:21-27

Humber F, Andriamahaino ET, Beriziny T, Botosoamananto R, Godley BJ, Gough C, Pedron S, Ramahery V, Broderick AC (2017) Assessing the small-scale shark fishery of Madagascar through community-based monitoring and knowledge. Fish Res 186:131143. https://doi.org/10.1016/j.fishres.2016.08.012

IUCN (2020) The IUCN red list of threatened species. Version 2020-2. https://www.iucnredlist.org. Downloaded on 28 November 2020

Jabado RA (2018) The fate of the most threatened order of elasmobranchs: shark-like batoids (Rhinopristiformes) in the Arabian Sea and adjacent waters. Fish Res 204:448-457. https://doi.org/10.1016/ j.fishres.2018.03.022

Jabado RW, Kyne PM, Pollom RA, Ebert DA, Simpfendorfer CA, Ralph GM, Al Dhaheri SS, Akhilesh KV, Ali K, Ali MH, Al Mamari TMS, Bineesh KK, El Hassan IS, Fernando D, Grandcourt EM, Khan MM, Moore ABM, Owfi F, Robinson DP, Romanov E, Soares A-L, Spaet JLY, Tesfamichael D, Valinassab T, Dulvy NK (2018) Troubled waters: threats and extinction risk of the sharks, rays and chimaeras of the Arabian Sea and adjacent waters. Fish Fish 19:1043-1062. https://doi.org/10.1111/faf.12311

Kyne PM, Jabado RW, Rigby CL, Dharmadi GMA, Pollock CM, Herman KB, Cheok J, Ebert DA, Simpfendorfer CA, Dulvy NK (2020) The thin edge of the wedge: extremely high extinction rick in wedgefishes and giant guitarfishes. Aquat Conserv 30(7):13371361. https://doi.org/10.1002/aqc.3331

Last PR, Compagno LJV, Nakaya K (2004) Rhinobatos nudidorsalis, a new species of shovelnose ray (Batoidea: Rhinobatidae) from the Mascarene Ridge, central Indian Ocean. Ichthyol Res 51:153-158. https://doi.org/10.1007/s10228-004-0211-0

Last PR, Séret B, Naylor GJP (2016a) A new species of guitarfish, Rhinobatos borneensis sp. nov. with a redefinition of the familylevel classification in the order Rhinopristiformes (Chondrichthyes: Batoidea). Zootaxa 4117(4):451-475. https://doi.org/10.11646/ zootaxa.4117.4.1

Last PR, Henderson AC, Naylor GJP (2016b) Acroteriobatus omanensis (Batoidea: Rhinobatidae), a new guitarfish from the Gulf of Oman. Zootaxa 4144(2):276-286. https://doi.org/10.11646/zootaxa.4144. 2.9

Linck HF (1790) Versuch einer Eintheilung der Fische nach den Zähnen. Mag Neueste Phys Naturgesch 6(3):28-38

Manilo LG, Bogorodsky SV (2003) Taxonomic composition, diversity and distribution of coastal fishes of the Arabian Sea. J Ichthyol 43(1):75-149

Maugé AL (1967) Contribution préliminaire à l'inventaire ichtyologique de la région de Tuléar. Ann Fac Sci Univ Madagascar 5:215-246

Moore ABM (2017) Are guitarfishes the next sawfish? Extinction risk and an urgent call for conservation action. Endanger Species Res 34: 75-88. https://doi.org/10.3354/esr00830

Müller J, Henle FGJ (1841) Systematische Beschreibung der Plagiostomen. Veit und Comp, Berlin 200 pp

Nair RV, Lal Mohan RS (1973) On a new deep sea skate, Rhinobatos variegatus, with notes on the deep sea sharks Halaelurus hispidus, Eridacnis radcliffei and Eugaleus omanensis from the Gulf of Mannar. Senckenberg Biol 54(1/3):71-80
Norman JR (1926) A synopsis of the rays of the family Rhinobatidae, with a revision of the genus Rhinobatus. Proc Zool Soc London 96(4):941-982. https://doi.org/10.1111/j.1096-3642.1926.tb02228.

Pollom R, Bennett R, Fernando S, Gledhill K, Kuguru B, Sink K (2019) Acroteriobatus leucospilus. The IUCN red list of threatened species 2019: e.T161555A124505883. https://doi.org/10.2305/IUCN.UK. 2019-3.RLTS.T161555A124505883.en

Randall JE, Compagno LJV (1995) A review of the guitarfishes of the genus Rhinobatos (Rajiformes: Rhinobatidae) from Oman, with description of a new species. Raffles Bull Zool 43(2):289-298

Regan CT (1908) A collection of fishes from the coasts of Natal, Zululand, and Cape Colony. Ann Natal Mus 1(3):241-255

Sabaj MH (ed) (2019) Standard symbolic codes for institutional resource collections in herpetology and ichthyology: an online reference, version 7.1 (21 March 2019). Available from http://www.asih.org/ (accessed 11 March 2020)

Séret B (in press) Chondrichthyan fishes (sharks, rays and chimaeras) of Madagascar. In: Goodman S, Cooke A (eds) The natural history of Madagascar. Princeton University Press, Princeton

Séret B, de Carvalho MR (in press) Family Rhinobatidae, guitarfishes and shovelnose rays. In: Heemstra P, Heemstra E, Ebert DA, Holleman $\mathrm{W}$, Randall J (eds) The coastal fishes of the western Indian Ocean. A longstanding vision realized. Vol. 1, pp 179-188, plates 58-61, pp 655-658

Séret B, Last PR, Naylor GJP (2016) Guitarfishes, family Rhinobatidae. In: Last PR, White WT, de Carvalho MR, Séret B, Stehmann MFW, Naylor GJP (eds) Rays of the world. CSIRO Publishing, Melbourne, pp 77-109

Smith JLB (1961) The sea fishes of southern Africa, 4th edn. Central News Agency, Ltd., South Africa 573 pp

Wallace JH (1967) The batoid fishes of the east coast of Southern Africa. Part I: sawfishes and guitarfishes. Invest Rep Oceanogr Res Inst Durban 15:1-32

Weigmann S (2016) Annotated checklist of the living sharks, batoids and chimaeras (Chondrichthyes) of the world, with a focus on biogeographical diversity. J Fish Biol 88(3):837-1037. https://doi.org/10. $1111 / \mathrm{jfb} .12874$

Weigmann S (2017) Reply to Borsa (2017): Comment on 'Annotated checklist of the living sharks, batoids and chimaeras (Chondrichthyes) of the world, with a focus on biogeographical diversity by Weigmann (2016)'. J Fish Biol 90(4):1176-1181. https://doi.org/10.1111/jfb.13234

Weigmann S, Stehmann MFW, Thiel R (2013) Planonasus parini n. g. and n. sp., a new genus and species of false cat sharks (Carchariniformes, Pseudotriakidae) from the deep northwestern Indian Ocean off Socotra Islands. Zootaxa 3609(2):163-181. https://doi.org/10.11646/zootaxa.3609.2.3

Weigmann S, Stehmann MFW, Thiel R (2015) Okamejei ornata n. sp., a new deep-water skate (Elasmobranchii, Rajidae) from the northwestern Indian Ocean off Socotra Islands. Deep-Sea Res Pt II 115: 18-29. https://doi.org/10.1016/j.dsr2.2013.09.005

Yan HF, Kyne PM, Jabado RW, Leeney RH, Davidson LNK, Derrick DH, Finucci B, Freckleton RP, Fordham SV, Dulvy NK (2021) Overfishing and habitat loss drive range contraction of iconic marine fishes to near extinction. Sci Adv 7(7):eabb6026. https://doi.org/10. 1126/sciadv.abb6026

Publisher's note Springer Nature remains neutral with regard to jurisdictional claims in published maps and institutional affiliations. 